\title{
FIRST-ORDER ASYMPTOTIC THEORY FOR PARAMETRIC MISSPECIFICATION TESTS OF GARCH MODELS
}

\author{
ANDREEA G. HaLUnga \\ University of Exeter \\ Chris D. ORME \\ University of Manchester
}

\begin{abstract}
This paper develops a framework for the construction and analysis of parametric misspecification tests for generalized autoregressive conditional heteroskedastic $(\mathrm{GARCH})$ models, based on first-order asymptotic theory. The principal finding is that estimation effects from the correct specification of the conditional mean (regression) function can be asymptotically nonnegligible. This implies that certain procedures, such as the asymmetry tests of Engle and $\mathrm{Ng}$ (1993, Journal of Finance 48, 1749-1777) and the nonlinearity test of Lundbergh and Teräsvirta (2002, Journal of Econometrics 110, 417-435), are asymptotically invalid. A second contribution is the proposed use of alternative tests for asymmetry and/or nonlinearity that, it is conjectured, should enjoy improved power properties. A Monte Carlo study supports the principal theoretical findings and also suggests that the new tests have fairly good size and very good power properties when compared with the Engle and Ng (1993) and Lundbergh and Teräsvirta (2002) procedures.
\end{abstract}

\section{INTRODUCTION}

A great deal of research has been undertaken on modeling volatility clustering in financial and economic time series, in which the generalized autoregressive conditional heteroskedastic (GARCH) model of Bollerslev (1986) represents a benchmark specification. The subsequent literature has provided generalizations by, e.g., allowing for asymmetric and/or nonlinear behavior. Prominent among these are the exponential GARCH (EGARCH) model of Nelson (1991), the GJR

\footnotetext{
We are grateful to three referees for their insightful comments, which greatly improved the exposition of this paper. Our thanks, also, to Ralf Becker, James Davidson, Len Gill, and Alastair Hall for constructive comments and/or discussions and to seminar participants at Cambridge, Manchester, and the 2007 Econometric Society European Meetings. The standard disclaimer applies. The first author's research is part of her Ph.D. thesis and was supported by a University Research Studentship and an Overseas Research Studentship, both of which are gratefully acknowledged. Address correspondence to Andreea G. Halunga, Department of Economics, University of Exeter, Streatham Court, Rennes Drive, Exeter EX4 4PU, United Kingdom; e-mail: a.g.halunga@exeter.ac.uk.
} 
model of Glosten, Jagannathan, and Runkle (1993), the threshold GARCH (TGARCH) model of Zakoïan (1994), and the smooth transition GARCH (STGARCH) model of Hagerud (1997) and Gonzalez-Rivera (1998).

Notwithstanding these developments, the parametric GARCH model remains a popular choice among applied workers. Therefore, and as noted by Lundbergh and Teräsvirta (2002), it is important to perform misspecification tests to assess the adequacy of the parametric model being employed. Developing misspecification tests has not been a neglected area of research. Bollerslev (1986) suggested a natural score type test for testing a GARCH model against a higher order GARCH model. Asymmetry tests were proposed by Engle and $\mathrm{Ng}$ (1993), and these are now widely used in empirical finance. Li and Mak (1994) constructed a test for the adequacy of a $\operatorname{GARCH}(p, q)$ model with a null hypothesis that the squared standardized error process is serially uncorrelated. Lundbergh and Teräsvirta (2002) proposed tests of (1) no remaining autoregressive conditional heteroskedasticity (ARCH) in standardized errors, (2) linearity, and (3) parameter constancy. All of these procedures are important inferential tools for empirical researchers who are interested in obtaining accurate forecasts of financial volatility to make the appropriate decisions on portfolio selection, asset management, or pricing derivative assets.

However, in this paper it is argued that, on closer inspection, the standard firstorder theory employed to justify the asymptotic validity of such procedures has sometimes been misinterpreted. To establish this, a unifying framework for the construction and analysis of parametric misspecification tests in GARCH models, based on the conditional moment principle and first-order asymptotic theory, is developed. This provides a useful contribution in at least two respects.

First, and most significantly, the theory predicts that the limit null distribution of the relevant test indicators must take account of asymptotically nonnegligible effects from the estimated conditional mean (regression) parameters in the null $\operatorname{GARCH}(p, q)$ model. (The importance of such estimation effects was addressed by Durbin, 1970, when testing for serial correlation with lagged dependent variables.) This issue has been apparently overlooked in the GARCH testing literature because in the null $\operatorname{GARCH}(p, q)$ model, under conditional symmetry of the errors, the estimated conditional mean parameters are asymptotically orthogonal to estimated conditional heteroskedasticity parameters. In particular, and because of this orthogonality, it appears that the conditional mean estimation effects have been simply (but erroneously) assumed away, e.g., by Engle and Ng (1993) and Lundbergh and Teräsvirta (2002). ${ }^{1}$

The second contribution proposes "new" tests for asymmetry and/or nonlinearity. It is conjectured that these test procedures should have better power properties against the types of alternative models considered by both Engle and $\mathrm{Ng}$ (1993) and Lundbergh and Teräsvirta (2002) in their Monte Carlo experiments, because their construction takes into account the recursive nature of the conditional heteroskedasticity (whereas the test procedures of Engle and $\mathrm{Ng}, 1993$, and Lundbergh and Teräsvirta, 2002, do not). The results of a small Monte Carlo 
study reveal that the new tests do indeed have good size properties and very good power when compared with the tests of Engle and Ng (1993) and Lundbergh and Teräsvirta (2002).

The paper is organized as follows, with supporting lemmas and propositions, together with all proofs, relegated to Appendixes. Section 2 describes the null GARCH model and briefly discusses quasi-maximum likelihood (QML) estimation. Section 3 describes a framework for constructing a particular class of parametric misspecification tests. In Section 4 the tests proposed by Lundbergh and Teräsvirta (2002) and Engle and $\mathrm{Ng}$ (1993) are reviewed, and new asymptotically valid tests for asymmetry and nonlinearity are introduced. Section 5 presents some Monte Carlo evidence in support of the theoretical findings, and Section 6 concludes.

\section{THE NULL GARCH $(p, q)$ MODEL}

\subsection{Assumptions and Estimation Framework}

The regression model for the variable of interest, $y_{t}$, is defined as

$y_{t}=m\left(\mathbf{w}_{t} ; \boldsymbol{\varphi}_{0}\right)+\varepsilon_{0 t}, \quad t=1, \ldots, T$,

where $\mathbf{w}_{t}=\left(\mathbf{y}_{t-1}^{\prime}, \mathbf{z}_{t}^{\prime}\right), \mathbf{y}_{t-1}=\left(1, y_{t-1}, \ldots, y_{t-l}\right)^{\prime} \in \mathfrak{R}^{l+1}, \mathbf{z}_{t}=\left(z_{t 1}, \ldots, z_{t k}\right)^{\prime} \in$ $\mathfrak{R}^{k}$ are exogenous variables, $\boldsymbol{\varphi}_{0}=\left(\varphi_{01}, \ldots, \varphi_{0 r}\right)^{\prime}$ is the true parameter vector, and the conditional mean (regression) function, $m\left(\mathbf{w}_{t} ; \boldsymbol{\varphi}_{0}\right)$, is possibly nonlinear. ${ }^{2}$ The error $\left\{\varepsilon_{0 t}, \mathcal{F}_{t}\right\}$, where $\mathcal{F}_{t-1}=\sigma\left(\left(y_{t-1}, \mathbf{z}_{t}^{\prime}\right),\left(y_{t-2}, \mathbf{z}_{t-1}^{\prime}\right), \ldots\right)$, is a martingale difference sequence given by

$\varepsilon_{0 t}=\xi_{t} h_{0 t}^{1 / 2}$

where the standardized error process, $\xi_{t}$, is an independent and identically distributed (i.i.d.) sequence with mean zero and variance one and we define $k_{c}=$ $\mathrm{E}\left[\xi_{t}^{4}\right]$ and $v_{c}=\mathrm{E}\left[\xi_{t}^{3}\right]$, both finite constants. The conditional variance is specified as

$$
\begin{aligned}
h_{0 t} & =\boldsymbol{\eta}_{0}^{\prime} \mathbf{s}_{0, t-1} \\
& =\alpha_{00}+A_{0}(L) \varepsilon_{0 t}^{2}+B_{0}(L) h_{0 t},
\end{aligned}
$$

where $\mathbf{s}_{0, t-1}=\left(1, \varepsilon_{0, t-1}^{2}, \ldots, \varepsilon_{0, t-q}^{2}, h_{0, t-1}, \ldots, h_{0, t-p}\right)^{\prime}, \boldsymbol{\eta}_{0}=\left(\alpha_{00}, \alpha_{01}, \ldots, \alpha_{0 q}\right.$, $\left.\beta_{01}, \ldots, \beta_{0 p}\right)^{\prime}, A_{0}(L)=\alpha_{01} L+\cdots+\alpha_{0 q} L^{q}$, and $B_{0}(L)=\beta_{01} L+\cdots+\beta_{0 p} L^{p}$.

The preceding process is defined for the true parameter $\boldsymbol{\theta}_{0}=\left(\boldsymbol{\varphi}_{0}^{\prime}, \boldsymbol{\eta}_{0}^{\prime}\right)^{\prime}$, and correspondingly the model for the unknown parameter vector $\boldsymbol{\theta}=\left(\boldsymbol{\varphi}^{\prime}, \boldsymbol{\eta}^{\prime}\right)^{\prime}$ is defined as 


$$
\begin{aligned}
y_{t} & =m\left(\mathbf{w}_{t} ; \boldsymbol{\varphi}\right)+\varepsilon_{t}, \quad t=1, \ldots, T, \\
h_{t} & =\boldsymbol{\eta}^{\prime} \mathbf{s}_{t-1} \\
& =\alpha_{0}+A(L) \varepsilon_{t}^{2}+B(L) h_{t} \\
& =a_{t}+B(L) h_{t},
\end{aligned}
$$

where $a_{t}=\alpha_{0}+A(L) \varepsilon_{t}^{2}=\alpha_{0}+\sum_{k=1}^{q} \alpha_{k} \varepsilon_{t-k}^{2}$. The following assumptions ensure the identifiability, stationarity, and ergodicity of the preceding process.

\section{Assumptions A.}

1. The parameter space, $\boldsymbol{\Theta}$, is compact, and $\boldsymbol{\theta}_{0}$ lies in the interior of $\Theta$.

2. The elements of $\left(y_{t}, \mathbf{z}_{t}^{\prime}\right)$ are strictly stationary and ergodic, and $m\left(\mathbf{w}_{t} ; \boldsymbol{\varphi}\right)$ is continuous and $\mathcal{F}_{t-1}$ measurable for all $\varphi \in \Theta$.

3. (a) All the roots of $1-A(z)-B(z)=0$ lie outside the unit circle;

(b) the parameter space is constrained such that $0<\lambda \leq \min \left\{\eta_{l}\right\} \leq \max \left\{\eta_{l}\right\}$ $<\Lambda, l=1, \ldots, p+q+1$, where $\lambda$ and $\Lambda$ are independent of $\boldsymbol{\theta}$;

(c) the polynomials $A(z)$ and $1-B(z)$ are coprimes.

As in Ling and McAleer (2003), Assumption A3(a) is a stationarity assumption imposed over the whole parameter space. Notice that, with A3(b), this implies that roots of $1-B(z)=0$ lie outside the unit circle. Thus, in addition to A3(b), which restricts the parameter space so that zero values in $\boldsymbol{\eta}$ are ruled out, $\sum_{j=1}^{p} \beta_{j}<1$. These restrictions are also imposed on $\Theta$ by Berkes, Horváth, and Kokoszka (2003) and are employed here because they afford uniform convergence of second derivatives of the log-likelihood over $\Theta$, removing the need for third derivatives, thus greatly simplifying the algebra required to justify the substantive contribution. $^{3}$

Given Assumptions A3(a) and (b), the process for $h_{t}$ has the following representation:

$h_{t}^{\infty}=(1-B(L))^{-1} a_{t}=\sum_{i=0}^{\infty} \psi_{i} a_{t-i}$,

where $(1-B(L))^{-1}=\sum_{i=0}^{\infty} \psi_{i} L^{i}$, with $\psi_{0}=1, \psi_{i}>0$ and satisfying $\psi_{i}=$ $\sum_{j=1}^{p} \beta_{j} \psi_{i-j}$, with $\psi_{s}=0, s<0,0<\sum_{i=0}^{\infty} \psi_{i}=\left(1-\sum_{j=1}^{p} \beta_{j}\right)^{-1}<\infty$. The coefficients, $\psi_{i}$, decay exponentially fast, and there exist constants $K>0$ and $0<\rho<1$, independent of $\boldsymbol{\theta}$, such that $\psi_{i} \leq K \rho^{i}$. Then, as in Ling and McAleer (2003), but under (1), Assumption A2, and $h_{0 t}=h_{t}^{\infty}\left(\boldsymbol{\theta}_{0}\right)$, it can be shown that $\left\{\varepsilon_{0 t}, h_{0 t}\right\}$ is strictly stationary and ergodic.

Asymptotic theory for GARCH models has been considered by several authors. For example, Ling and McAleer (2003) required that $\mathrm{E}\left(\varepsilon_{0 t}^{6}\right)<\infty$ to ensure asymptotic normality of the QML estimator in the ARMA-GARCH model. 
Furthermore, Chan and McAleer $(2002,2003)$ argued that the results in Ling and McAleer (2003) also hold for a smooth transition autoregressive-GARCH (STAR-GARCH) model. Berkes et al. (2003) established the consistency and asymptotic normality of the QML estimator, under weaker moment assumptions, in the pure $\operatorname{GARCH}(p, q)$ model, these being $\mathrm{E}\left[\xi_{t}^{2}\right]=1$ for consistency and $\mathrm{E}\left[\xi_{t}^{4}\right]<\infty$ for asymptotic normality. Francq and Zakoïan (2004) established consistency and asymptotic normality of the QML estimator in both a pure GARCH and an ARMA-GARCH model under further weakened conditions that, in the pure GARCH model, allow zero values in the parameter space. Recently, Francq and Zakoïan (2007) have in addition extended the analysis to the case where the true parameter, $\boldsymbol{\eta}_{0}$, might also contain zero values. This assumption, however, precludes asymptotic normality for the quasi maximum likelihood estimator (QMLE). All these authors assume, as here, that the $\xi_{t}$ are i.i.d. Therefore, although it is possible that the assumptions employed in this paper could be weakened, it should be noted that the regression specification in (1) is more general than that employed in the literature referred to earlier and the corresponding assumptions employed are, nonetheless, sufficient and (importantly) permit a relatively straightforward justification of the required first-order asymptotic theory, without obfuscating the principal issue that is addressed in the paper. In practice, and following Weiss (1986), ${ }^{4}$ the existence of moments is assumed when required as follows, where $\|\cdot\|$ denotes the euclidean norm.

\section{Assumptions B.}

1. $\mathrm{E}\left|\varepsilon_{0 t}\right|^{4(1+s)}<\infty$ for some $s>0$ and all $t$.

2. $\mathrm{E}\left|m\left(\mathbf{w}_{t} ; \boldsymbol{\varphi}\right)-m\left(\mathbf{w}_{t}, \boldsymbol{\varphi}_{0}\right)\right|^{2}>0$, for all $\boldsymbol{\varphi} \neq \boldsymbol{\varphi}_{0}$.

3. $m\left(\mathbf{w}_{t} ; \boldsymbol{\varphi}\right)$ is at least twice continuously differentiable in $\boldsymbol{\varphi}$, with, for all $t$,

(a) $\sup _{\boldsymbol{\theta}}\left|m\left(\mathbf{w}_{t} ; \boldsymbol{\varphi}\right)\right|^{4(1+s)}<B\left(\mathbf{w}_{t}\right)$, with $\mathrm{E}\left[B\left(\mathbf{w}_{t}\right)\right]<\infty$, for some $s>0$;

(b) $\left.\operatorname{E}_{\sup }\left\|\operatorname{suc}_{t}^{r} \partial m\left(\mathbf{w}_{t-i} ; \boldsymbol{\varphi}\right) / \partial \boldsymbol{\varphi}\right\|^{2}\right]<\infty, r=0,2$, and all $i \geq 0$;

(c) $\mathrm{E}\left[\sup _{\boldsymbol{\theta}}\left\|\varepsilon_{t}^{r} \partial^{2} m\left(\mathbf{w}_{t-i} ; \boldsymbol{\varphi}\right) / \partial \boldsymbol{\varphi} \partial \boldsymbol{\varphi}^{\prime}\right\|^{2}\right]<\infty, r=0,1$, and all $i \geq 0$.

The (average) quasi log-likelihood, conditional on available presample values $\tilde{\mathbf{y}}=\left(y_{0}, \ldots, y_{1-l}\right)^{\prime}$ is (ignoring constants)

$L_{T}(\boldsymbol{\theta})=\frac{1}{T} \sum_{t=1}^{T} l_{t}(\boldsymbol{\theta}), \quad l_{t}(\boldsymbol{\theta})=-\frac{1}{2}\left[\ln \left(h_{t}\right)+\frac{\varepsilon_{t}^{2}}{h_{t}}\right]$,

although the ensuing asymptotic analysis does not restrict $\xi_{t}$ to be normally distributed; see Bollerslev (1986). Note that (5) is not only conditional on available presample values, $\tilde{\boldsymbol{y}}$, from which $\varepsilon_{t}, t=1, \ldots, T$, can be constructed, but also on $\tilde{\boldsymbol{\varepsilon}}_{0}=\left(\varepsilon_{0}^{2}, \ldots, \varepsilon_{1-q}^{2}, h_{0}, \ldots, h_{1-p}\right)^{\prime}$, from which $h_{t}$ can be constructed using (4). However, $\varepsilon_{t}$ and the process $h_{t}, t \leq 0$, are unobserved. To simplify the algebra and asymptotic theory, it is assumed (in addition) that presample 
observations $\mathbf{w}_{0}, \ldots, \mathbf{w}_{1-q}$ are also available and that $h_{t}=0$ for all $t \leq 0 .{ }^{5}$ The simplifications in the analysis derive from the fact that now $h_{t}$ can be expressed as $h_{t}=\sum_{i=0}^{t-1} \psi_{i} a_{t-i}=\sum_{i=0}^{t-1}\left\{B^{*}(L)\right\}^{i} a_{t-i}, t=1, \ldots, T$, where $B^{*}(L)=\beta_{1}+\beta_{2} L+$ $\cdots+\beta_{p} L^{p-1}$. (In practice, though, and for all inferential procedures described in this paper, a constant value can be chosen for $\tilde{\boldsymbol{\varepsilon}}_{0}$ to generate $h_{t}, t=1, \ldots, T$ ). The unknown parameters can be estimated jointly by QML estimation of (5). Throughout, the estimated parameter vector will be denoted $\hat{\boldsymbol{\theta}}^{\prime}=\left(\hat{\boldsymbol{\varphi}}^{\prime}, \hat{\boldsymbol{\eta}}^{\prime}\right)$.

The unobserved log-likelihood function, conditioning on the infinite history of all past observations $\left(\mathbf{w}_{0}^{\prime}, \mathbf{w}_{-1}^{\prime}, \mathbf{w}_{-2}^{\prime}, \ldots\right)^{\prime}$, is $L_{T}^{\infty}(\boldsymbol{\theta})=\frac{1}{T} \sum_{t=1}^{T} l_{t}^{\infty}(\boldsymbol{\theta}) \neq L_{T}(\boldsymbol{\theta})$, with $l_{t}^{\infty}(\boldsymbol{\theta})=-\frac{1}{2}\left(\ln \left(h_{t}^{\infty}\right)+\left(\varepsilon_{t}^{2} / h_{t}^{\infty}\right)\right)$ and score vector contributions of $\mathbf{d}_{\boldsymbol{\theta} t}^{\infty}(\boldsymbol{\theta})=$ $\frac{\partial l_{t}^{\infty}(\boldsymbol{\theta})}{\partial \boldsymbol{\theta}}$, where $\mathbf{d}_{\boldsymbol{\theta} t}^{\infty}(\boldsymbol{\theta})=\left(\mathbf{d}_{\boldsymbol{\varphi} t}^{\infty}(\boldsymbol{\theta})^{\prime}, \mathbf{d}_{\boldsymbol{\eta} t}^{\infty}(\boldsymbol{\theta})^{\prime}\right)^{\prime}$ in an obvious manner. Assuming $L_{T}^{\infty}(\boldsymbol{\theta})$ and $L_{T}(\boldsymbol{\theta})$ are both twice continuously differentiable in $\boldsymbol{\theta}$, define $\mathbf{d}_{\boldsymbol{\theta} t}(\boldsymbol{\theta})=\frac{\partial l_{t}(\boldsymbol{\theta})}{\partial \boldsymbol{\theta}}, \mathbf{D}_{\boldsymbol{\theta} T}(\boldsymbol{\theta})=T^{-1} \sum_{t=1}^{T} \mathbf{d}_{\boldsymbol{\theta} t}(\boldsymbol{\theta}), \mathbf{P}_{\boldsymbol{\theta} \boldsymbol{\theta} T}(\boldsymbol{\theta})=-T^{-1} \sum_{t=1}^{T} \frac{\partial \mathbf{d}_{\boldsymbol{\theta} t}(\boldsymbol{\theta})}{\partial \boldsymbol{\theta}^{\prime}}$, and, correspondingly, $\mathbf{D}_{\boldsymbol{\theta} T}^{\infty}(\boldsymbol{\theta})$ and $\mathbf{P}_{\boldsymbol{\theta} \boldsymbol{\theta} T}^{\infty}(\boldsymbol{\theta})$ for the unobserved $L_{T}^{\infty}(\boldsymbol{\theta})$. By introducing the unobserved log-likelihood, the methodology of Ling and McAleer (2003), Berkes et al. (2003), and Francq and Zakoïan (2004) is followed, whereby it is established that $\hat{\boldsymbol{\theta}}=\arg \max _{\boldsymbol{\theta}} L_{T}(\boldsymbol{\theta})$ has exactly the same first-order asymptotic properties as $\hat{\boldsymbol{\theta}}^{\infty}=\arg \max _{\boldsymbol{\theta}} L_{T}^{\infty}(\boldsymbol{\theta})$, with the latter being fairly easy to verify.

To develop these arguments, it will be useful to illustrate, and distinguish between, the various unobserved and observed quantities associated with $L_{T}^{\infty}(\boldsymbol{\theta})$ and $L_{T}(\boldsymbol{\theta})$, respectively, based on the assumed initial start-up values embodied in $\tilde{\boldsymbol{\varepsilon}}_{0}$. Specifically, the unobserved scores are $\mathbf{D}_{\boldsymbol{\varphi} T}^{\infty}(\boldsymbol{\theta})=T^{-1} \sum_{t=1}^{T} \times$ $\left\{\frac{\varepsilon_{t} \mathbf{f}_{t}}{h_{t}^{\infty}}+\frac{1}{2}\left(\frac{\varepsilon_{t}^{2}}{h_{t}^{\infty}}-1\right) \mathbf{c}_{t}^{\infty}\right\}, \mathbf{D}_{\boldsymbol{\eta} T}^{\infty}(\boldsymbol{\theta})=T^{-1} \frac{1}{2} \sum_{t=1}^{T}\left(\frac{\varepsilon_{t}^{2}}{h_{t}^{\infty}}-1\right) \mathbf{x}_{t}^{\infty}$, where $\mathbf{f}_{t}=\frac{\partial m\left(\mathbf{w}_{t} ; \boldsymbol{\varphi}\right)}{\partial \boldsymbol{\varphi}}$, and by exploiting the recursions $\frac{\partial h_{t}}{\partial \boldsymbol{\varphi}}=-2 \sum_{k=1}^{q} \alpha_{k} \varepsilon_{t-k} \mathbf{f}_{t-k}+B(L) \frac{\partial h_{t}}{\partial \boldsymbol{\varphi}}$ and $\frac{\partial h_{t}}{\partial \boldsymbol{\eta}}=$ $\mathbf{s}_{t-1}+B(L) \frac{\partial h_{t}}{\partial \boldsymbol{\eta}}$,

$\mathbf{c}_{t}^{\infty}=\frac{1}{h_{t}^{\infty}} \frac{\partial h_{t}^{\infty}}{\partial \boldsymbol{\varphi}}=-2 \frac{1}{h_{t}^{\infty}} \sum_{k=1}^{q} \alpha_{k}\left\{\sum_{i=0}^{\infty} \psi_{i} \varepsilon_{t-k-i} \mathbf{f}_{t-k-i}\right\}$

and

$\mathbf{x}_{t}^{\infty}=\frac{1}{h_{t}^{\infty}} \frac{\partial h_{t}^{\infty}}{\partial \boldsymbol{\eta}}=\frac{1}{h_{t}^{\infty}} \sum_{i=0}^{\infty} \psi_{i} \mathbf{s}_{t-1-i}^{\infty}$

where $\mathbf{s}_{t-1}^{\infty \prime}=\left(1, \varepsilon_{t-1}^{2}, \ldots, \varepsilon_{t-q}^{2}, h_{t-1}^{\infty}, \ldots, h_{t-p}^{\infty}\right)^{\prime}$. Given $\tilde{\boldsymbol{\varepsilon}}_{0}$, the corresponding observed score $\mathbf{D}_{\boldsymbol{\theta} T}(\boldsymbol{\theta})$, associated with $L_{T}(\boldsymbol{\theta})$, can be expressed analogously but with

$$
\mathbf{c}_{t}=-2 \frac{1}{h_{t}} \sum_{k=1}^{q} \alpha_{k}\left\{\sum_{i=0}^{t-1} \psi_{i} \varepsilon_{t-k-i} \mathbf{f}_{t-k-i}\right\}
$$




$$
\begin{aligned}
& =-2 \frac{1}{h_{t}} \sum_{k=1}^{q} \alpha_{k}\left\{\sum_{i=0}^{t-1}\left\{B^{*}(L)\right\}^{i} \varepsilon_{t-i-k} \mathbf{f}_{t-i-k}\right\} \\
\mathbf{x}_{t} & =\frac{1}{h_{t}} \sum_{i=0}^{t-1} \psi_{i} \mathbf{s}_{t-1-i}=\frac{1}{h_{t}} \sum_{i=0}^{t-1}\left\{B^{*}(L)\right\}^{i} \mathbf{s}_{t-1-i}
\end{aligned}
$$

replacing $\mathbf{c}_{t}^{\infty}$ and $\mathbf{x}_{t}^{\infty}$, respectively. For example, in the $\operatorname{GARCH}(1,1)$ case $h_{t}=\sum_{i=0}^{t-1} \beta_{1}^{i}\left\{\alpha_{0}+\alpha_{1} \varepsilon_{t-1-i}^{2}\right\}, \quad \mathbf{c}_{t}=-2 h_{t}^{-1} \alpha_{1} \sum_{i=0}^{t-1} \beta_{1}^{i} \varepsilon_{t-1-i} \mathbf{f}_{t-1-i}, \quad$ and $\mathbf{x}_{t}=h_{t}^{-1} \sum_{i=0}^{t-1} \beta_{1}^{i} \mathbf{s}_{t-1-i}$, with $\mathbf{s}_{t-1}^{\prime}=\left(1, \varepsilon_{t-1}^{2}, h_{t-1}\right)$. In practice, however, $\mathbf{c}_{t}$ and $\mathbf{x}_{t}$ can be constructed using the recursions for $\frac{\partial h_{t}}{\partial \boldsymbol{\varphi}}$ and $\frac{\partial h_{t}}{\partial \boldsymbol{\eta}}$ described previously.

The consistency and asymptotic normality of the QMLE estimator $\hat{\boldsymbol{\theta}}=\arg \max \boldsymbol{\theta}$ $L_{T}(\boldsymbol{\theta})$ are presented in Section 2.2 together with a consistent variance-covariance matrix estimator.

\subsection{QML Estimation}

The following theorem establishes the consistency and asymptotic normality of $\hat{\boldsymbol{\theta}}$.

THEOREM 1. Given Assumptions $A$ and $B, \hat{\boldsymbol{\theta}} \stackrel{p}{\rightarrow} \boldsymbol{\theta}_{0}$ and

$$
\sqrt{T}\left(\hat{\boldsymbol{\theta}}-\boldsymbol{\theta}_{0}\right) \stackrel{d}{\rightarrow} N\left(\mathbf{0}, \mathbf{J}_{\boldsymbol{\theta} \boldsymbol{\theta}}^{-1} \mathbf{\Omega}_{\boldsymbol{\theta} \boldsymbol{\theta}} \mathbf{J}_{\boldsymbol{\theta} \boldsymbol{\theta}}^{-1}\right),
$$

where $\mathbf{J}_{\boldsymbol{\theta} \boldsymbol{\theta}}=-\mathrm{E}\left[\frac{\partial \boldsymbol{d}_{\boldsymbol{\theta} t}^{\infty}\left(\boldsymbol{\theta}_{0}\right)}{\partial \boldsymbol{\theta}^{\prime}}\right]$ and $\boldsymbol{\Omega}_{\boldsymbol{\theta} \boldsymbol{\theta}}=\mathrm{E}\left[\mathbf{d}_{\boldsymbol{\theta} t}^{\infty}\left(\boldsymbol{\theta}_{0}\right) \mathbf{d}_{\boldsymbol{\theta} t}^{\infty}\left(\boldsymbol{\theta}_{0}\right)^{\prime}\right]$ are both finite and positive definite with

$$
\mathbf{J}_{\boldsymbol{\theta} \boldsymbol{\theta}}=\left[\begin{array}{cc}
\mathbf{J}_{\varphi \varphi} & \mathbf{J}_{\eta \varphi}^{\prime} \\
\mathbf{J}_{\eta \varphi} & \mathbf{J}_{\eta \eta}
\end{array}\right]=\frac{1}{2} \mathrm{E}\left[\begin{array}{cc}
\mathbf{c}_{t}^{\infty} \mathbf{c}_{t}^{\infty \prime} & \mathbf{c}_{t}^{\infty} \mathbf{x}_{t}^{\infty \prime} \\
\mathbf{x}_{t}^{\infty} \mathbf{c}_{t}^{\infty \prime} & \mathbf{x}_{t}^{\infty} \mathbf{x}_{t}^{\infty \prime}
\end{array}\right]_{\boldsymbol{\theta}=\boldsymbol{\theta}_{0}}+\mathrm{E}\left[\begin{array}{cc}
\frac{1}{h_{t}^{\infty}} \mathbf{f}_{t} \mathbf{f}_{t}^{\prime} & \mathbf{0} \\
\mathbf{0} & \mathbf{0}
\end{array}\right]_{\boldsymbol{\theta}=\boldsymbol{\theta}_{0}}
$$

and

$$
\begin{aligned}
\boldsymbol{\Omega}_{\boldsymbol{\theta} \boldsymbol{\theta}}= & {\left[\begin{array}{ll}
\boldsymbol{\Omega}_{\boldsymbol{\varphi} \varphi} & \boldsymbol{\Omega}_{\boldsymbol{\eta} \varphi}^{\prime} \\
\boldsymbol{\Omega}_{\boldsymbol{\eta} \boldsymbol{\varphi}} & \boldsymbol{\Omega}_{\boldsymbol{\eta} \boldsymbol{\eta}}
\end{array}\right] } \\
= & \frac{\left(k_{c}-1\right)}{4} \mathrm{E}\left[\begin{array}{cc}
\mathbf{c}_{t}^{\infty} \mathbf{c}_{t}^{\infty \prime} & \mathbf{c}_{t}^{\infty} \mathbf{x}_{t}^{\infty \prime} \\
\mathbf{x}_{t}^{\infty} \mathbf{c}_{t}^{\infty \prime} & \mathbf{x}_{t}^{\infty} \mathbf{x}_{t}^{\infty \prime}
\end{array}\right]_{\boldsymbol{\theta}=\boldsymbol{\theta}_{0}} \\
& +\frac{v_{c}}{2} \mathrm{E}\left[\begin{array}{cc}
\frac{1}{\sqrt{h_{t}^{\infty}}} \mathbf{f}_{t} \mathbf{c}_{t}^{\infty \prime} & \frac{1}{\sqrt{h_{t}^{\infty}}} \mathbf{f}_{t} \mathbf{x}_{t}^{\infty \prime} \\
\frac{1}{\sqrt{h_{t}^{\infty}}} \mathbf{x}_{t}^{\infty} \mathbf{f}_{t}^{\prime} & \mathbf{0}
\end{array}\right]_{\boldsymbol{\theta}=\boldsymbol{\theta}_{0}}+\mathrm{E}\left[\begin{array}{cc}
\frac{1}{h_{t}^{\infty}} \mathbf{f}_{t} \mathbf{f}_{t}^{\prime} & \mathbf{0} \\
\mathbf{0} & \mathbf{0}
\end{array}\right]_{\boldsymbol{\theta}=\boldsymbol{\theta}_{0}} .
\end{aligned}
$$


Consistent standard errors follow from the next lemma, in which $\hat{\mathbf{C}}, \hat{\mathbf{X}}$, and $\hat{\mathbf{F}}$ are matrices with rows $\hat{\mathbf{c}}_{t}^{\prime}, \hat{\mathbf{x}}_{t}^{\prime}$, and $\hat{\mathbf{f}}_{t}^{\prime}$, respectively, and $\hat{\mathbf{H}}=\operatorname{diag}\left(\hat{\boldsymbol{\eta}}^{\prime} \hat{\mathbf{s}}_{t-1}\right)$, where "hats" denote evaluation at $\hat{\boldsymbol{\theta}}$.

LEMMA 1. Under Assumptions A and B,

(i) $\hat{\mathbf{\Omega}}_{\boldsymbol{\theta} \boldsymbol{\theta}}-\mathbf{\Omega}_{\boldsymbol{\theta \theta}}=o_{p}(1)$, where

$$
\begin{aligned}
& \hat{\mathbf{\Omega}}_{\boldsymbol{\theta} \boldsymbol{\theta}}= \frac{\left(\hat{k}_{c}-1\right)}{4} \frac{1}{T}\left[\begin{array}{cc}
\hat{\mathbf{C}}^{\prime} \hat{\mathbf{C}} & \hat{\mathbf{C}}^{\prime} \hat{\mathbf{X}} \\
\hat{\mathbf{X}}^{\prime} \hat{\mathbf{C}} & \hat{\mathbf{X}}^{\prime} \hat{\mathbf{X}}
\end{array}\right] \\
&+\frac{\hat{v}_{c}}{2} \frac{1}{T}\left[\begin{array}{cc}
\hat{\mathbf{F}}^{\prime} \hat{\mathbf{H}}^{-1 / 2} \hat{\mathbf{C}} & \hat{\mathbf{F}}^{\prime} \hat{\mathbf{H}}^{-1 / 2} \hat{\mathbf{X}} \\
\hat{\mathbf{X}}^{\prime} \hat{\mathbf{H}}^{-1 / 2} \hat{\mathbf{F}} & \mathbf{0}
\end{array}\right]+\frac{1}{T}\left[\begin{array}{cc}
\hat{\mathbf{F}}^{\prime} \hat{\mathbf{H}}^{-1} \hat{\mathbf{F}} & \mathbf{0} \\
\mathbf{0} & \mathbf{0}
\end{array}\right], \\
& \text { where } \hat{k}_{c}-1=\frac{1}{T} \sum_{t=1}^{T}\left(\frac{\varepsilon_{t}^{2}}{h_{t}}-1\right)_{\boldsymbol{\theta}=\hat{\boldsymbol{\theta}}}^{2} \text { and } \hat{v}_{c}=\frac{1}{T} \sum_{t=1}^{T}\left(\frac{\varepsilon_{t}}{\sqrt{h_{t}}}\right)_{\boldsymbol{\theta}=\hat{\boldsymbol{\theta}}}^{3} .
\end{aligned}
$$

(ii) $\hat{\mathbf{J}}_{\boldsymbol{\theta} \boldsymbol{\theta}}-\mathbf{J}_{\boldsymbol{\theta} \boldsymbol{\theta}}=o_{p}(1)$, where

$$
\hat{\mathbf{J}}_{\boldsymbol{\theta} \boldsymbol{\theta}}=\frac{1}{2} \frac{1}{T}\left[\begin{array}{cc}
\hat{\mathbf{C}}^{\prime} \hat{\mathbf{C}} & \hat{\mathbf{C}}^{\prime} \hat{\mathbf{X}} \\
\hat{\mathbf{X}}^{\prime} \hat{\mathbf{C}} & \hat{\mathbf{X}}^{\prime} \hat{\mathbf{X}}
\end{array}\right]+\frac{1}{T}\left[\begin{array}{cc}
\hat{\mathbf{F}}^{\prime} \hat{\mathbf{H}}^{-1} \hat{\mathbf{F}} & \mathbf{0} \\
\mathbf{0} & \mathbf{0}
\end{array}\right]
$$

Exploiting these results, and the method of proof, affords a framework in which to extend this asymptotic analysis to a specific class of misspecification tests.

\section{A CLASS OF ASYMPTOTICALLY VALID TEST PROCEDURES}

In this section, first-order asymptotic distribution results are developed for a class of parametric test statistics. The corresponding test procedures are derived from the conditional moment principle and are designed to detect misspecification in the null $\operatorname{GARCH}(p, q)$ error process, $h_{t}=\boldsymbol{\eta}^{\prime} \mathbf{s}_{t-1}$, while assuming a correct regression function specification, $m\left(\mathbf{w}_{t} ; \boldsymbol{\varphi}\right)$.

If the GARCH model is correctly specified, then it follows from (2) that

$\mathrm{E}\left[\left(\xi_{t}^{2}-1\right) \mid \mathcal{F}_{t-1}\right]=0$.

Therefore, misspecification tests of GARCH models can be constructed as tests of the following moment conditions:

$\mathrm{E}\left[\left(\xi_{t}^{2}-1\right) \mathbf{r}_{t}\left(\boldsymbol{\theta}_{0}\right)\right]=\mathbf{0}$,

where $\mathbf{r}_{t}\left(\boldsymbol{\theta}_{0}\right)$ is an $\mathcal{F}_{t-1}$ measurable function. The intuition here is that if the GARCH model is appropriate, then the squared standardized errors should be serially uncorrelated with any function of past information. ${ }^{6}$ 
Consistent with the notation introduced in the previous section, let $\mathbf{d}_{\boldsymbol{\pi} t}(\boldsymbol{\theta})=$ $\left(\left(\varepsilon_{t}^{2} / h_{t}\right)-1\right) \mathbf{r}_{t}(\boldsymbol{\theta})$, where the (test) variables in $\mathbf{r}_{t}$ will in general depend upon past history and specifically the process $h_{t}$. For example, $\mathbf{r}_{t}(\boldsymbol{\theta})$ could derive from a (quasi) score principle in which $\pi$ denotes the unknown parameter vector in the alternative model, say, $h_{t}^{a}$, and $H_{0}: \boldsymbol{\pi}=\mathbf{0}$ is under test. In this case, and ignoring irrelevant factors of proportionality, $\mathbf{r}_{t}(\boldsymbol{\theta})=\left[\frac{1}{h_{t}^{a}} \frac{\partial h_{t}^{a}}{\partial \boldsymbol{\pi}}\right]_{\boldsymbol{\pi}=\mathbf{0}}$; see Section 4.2. Therefore, as with $\mathbf{c}_{t}$ and $\mathbf{x}_{t}$, let $\mathbf{r}_{t}^{\infty}$ be the test variable constructed using $h_{t}^{\infty}$.

To test the null of (8), the generic conditional moment test indicator is constructed as

$\mathbf{D}_{\boldsymbol{\pi} T}(\hat{\boldsymbol{\theta}})=\frac{1}{T} \sum_{t=1}^{T}\left[\left(\frac{\hat{\varepsilon}_{t}^{2}}{\hat{h}_{t}}-1\right) \hat{\mathbf{r}}_{t}\right]=\frac{1}{T} \hat{\mathbf{R}}^{\prime} \hat{\boldsymbol{\vartheta}}$

where the matrix $\mathbf{R}$ has rows $\mathbf{r}_{t}^{\prime}=\mathbf{r}_{t}(\boldsymbol{\theta})^{\prime}, \hat{\boldsymbol{\vartheta}}$ is the vector with typical element $\left\{\frac{\hat{\varepsilon}_{t}^{2}}{\hat{h}_{t}}-1\right\}$, and "hats" denote that everything is evaluated at the consistent null parameter estimator, $\hat{\boldsymbol{\theta}}$. It should be noted that tests for nonlinearity and/or asymmetry, discussed in Section 4, are special cases. Assessing the statistical significance of (9), which requires estimation only under the null GARCH model, provides the basis for a test procedure.

It is not being claimed that such procedures are consistent in the sense of rejecting any departure from the null model when the null hypothesis is false. Given the framework set out in this paper, the general results of Godfrey and Orme (1996) could be employed to suggest alternatives against which tests based on (9), for a given choice of $\mathbf{r}_{t}(\boldsymbol{\theta})$, may be relatively insensitive. On the other hand, the conditional moment framework suggests that the Newey (1985) results can be exploited to determine the choice of $\mathbf{r}_{t}(\boldsymbol{\theta})$ that will provide optimal local power against particular forms of misspecification. Such issues are not the primary focus of the current paper, however.

The following theorem provides sufficient conditions under which the familiar limit distribution for $\sqrt{T} \mathbf{D}_{\pi T}(\hat{\boldsymbol{\theta}})$ applies. $^{7}$

THEOREM 2. Suppose that, in addition to Assumptions A and B, the following conditions are satisfied:

(i) $\sum_{t} \operatorname{Esup}_{\boldsymbol{\theta}}\left|\varepsilon_{t}\right|^{l}\left\|\mathbf{r}_{t}^{\infty}-\mathbf{r}_{t}\right\|=O(1), l=0,2$;

(ii) $\operatorname{Esup}_{\boldsymbol{\theta}}\left\|\mathbf{r}_{t}^{\infty}\right\|^{2}<\infty$, for all $t$;

(iii) $\operatorname{Esup}_{\boldsymbol{\theta}}\left\|\varepsilon_{t}^{l} \frac{\partial \mathbf{r}_{t}^{\infty}}{\partial \boldsymbol{\theta}}\right\|<\infty, l=0,2$, for all $t$.

Then

$\sqrt{T} \mathbf{D}_{\pi T}(\hat{\boldsymbol{\theta}}) \stackrel{d}{\rightarrow} N(\mathbf{0}, \boldsymbol{\Sigma})$, 
where

$\boldsymbol{\Sigma}=\mathbf{A} \boldsymbol{\Omega} \mathbf{A}^{\prime}$,

$\boldsymbol{\Omega}=\left[\begin{array}{ll}\boldsymbol{\Omega}_{\boldsymbol{\theta} \theta} & \mathbf{\Omega}_{\boldsymbol{\pi} \theta}^{\prime} \\ \mathbf{\Omega}_{\boldsymbol{\pi} \theta} & \mathbf{\Omega}_{\boldsymbol{\pi} \boldsymbol{\pi}}\end{array}\right], \quad \mathbf{A}=\left[-\mathbf{J}_{\boldsymbol{\pi} \theta} \mathbf{J}_{\boldsymbol{\theta} \boldsymbol{\theta}}^{-1}: \quad \mathbf{I}_{m}\right]$,

and $\mathbf{I}_{m}$ is the identity matrix of $\operatorname{rank} m=\operatorname{rank}\left(\mathbf{\Omega}_{\pi} \boldsymbol{\pi}\right)$,

$\boldsymbol{\Omega}_{\pi \varphi}=v_{c} \mathrm{E}\left[\frac{1}{\sqrt{h_{t}^{\infty}}} \mathbf{r}_{t}^{\infty} \mathbf{f}_{t}^{\prime}\right]_{\boldsymbol{\theta}=\boldsymbol{\theta}_{0}}+\frac{\left(k_{c}-1\right)}{2} \mathrm{E}\left[\mathbf{r}_{t}^{\infty} \mathbf{c}_{t}^{\infty \prime}\right]_{\boldsymbol{\theta}=\boldsymbol{\theta}_{0}}$,

$\boldsymbol{\Omega}_{\boldsymbol{\pi} \boldsymbol{\eta}}=\frac{\left(k_{c}-1\right)}{2} \mathrm{E}\left[\mathbf{r}_{t}^{\infty} \mathbf{x}_{t}^{\infty \prime}\right]_{\boldsymbol{\theta}=\boldsymbol{\theta}_{0}}$,

$\boldsymbol{\Omega}_{\pi \boldsymbol{\pi}}=\left(k_{c}-1\right) \mathrm{E}\left[\mathbf{r}_{t}^{\infty} \mathbf{r}_{t}^{\infty \prime}\right]_{\boldsymbol{\theta}=\theta_{0}}$,

and $\mathbf{J}_{\boldsymbol{\pi} \boldsymbol{\theta}}=\left[\mathbf{J}_{\boldsymbol{\pi} \varphi}: \mathbf{J}_{\boldsymbol{\pi} \boldsymbol{\eta}}\right]$ with

$\mathbf{J}_{\boldsymbol{\pi} \varphi}=-\mathrm{E}\left[\frac{\partial \mathbf{d}_{\pi t}^{\infty}\left(\boldsymbol{\theta}_{0}\right)}{\partial \boldsymbol{\varphi}^{\prime}}\right]=\mathrm{E}\left[\mathbf{r}_{t}^{\infty} \mathbf{c}_{t}^{\infty \prime}\right]_{\boldsymbol{\theta}=\boldsymbol{\theta}_{0}}$,

$\mathbf{J}_{\boldsymbol{\pi} \boldsymbol{\eta}}=-\mathrm{E}\left[\frac{\partial \mathbf{d}_{\boldsymbol{\pi} t}^{\infty}\left(\boldsymbol{\theta}_{0}\right)}{\partial \boldsymbol{\eta}^{\prime}}\right]=\mathrm{E}\left[\mathbf{r}_{t}^{\infty} \mathbf{x}_{t}^{\infty \prime}\right]_{\boldsymbol{\theta}=\boldsymbol{\theta}_{0}}$.

From the preceding result, the general form of the misspecification test statistic is the quadratic form

$T \mathbf{D}_{\pi T}(\hat{\boldsymbol{\theta}})^{\prime} \hat{\boldsymbol{\Sigma}}_{T}^{-1} \mathbf{D}_{\boldsymbol{\pi} T}(\hat{\boldsymbol{\theta}})$,

which has a $\chi_{m}^{2}$ limiting distribution under the null, where $\hat{\boldsymbol{\Sigma}}_{T}$ is any consistent estimator for $\Sigma$; i.e., $\hat{\Sigma}_{T}=\Sigma+o_{p}(1)$. Similar in spirit to Lemma 1, the following lemma gives an expression for $\hat{\Sigma}_{T}$.

LEMMA 2. Under Assumptions $A$ and $B$ and those of Theorem $2, \hat{\mathbf{A}} \hat{\mathbf{\Omega}} \hat{\mathbf{A}}^{\prime}-\boldsymbol{\Sigma}=$ $o_{p}(1)$ where

$\hat{\mathbf{\Omega}}=\left[\begin{array}{ll}\hat{\mathbf{\Omega}}_{\boldsymbol{\theta} \boldsymbol{\theta}} & \hat{\mathbf{\Omega}}_{\boldsymbol{\pi} \boldsymbol{\theta}}^{\prime} \\ \hat{\mathbf{\Omega}}_{\boldsymbol{\pi} \theta} & \hat{\mathbf{\Omega}}_{\boldsymbol{\pi} \boldsymbol{\pi}}\end{array}\right], \quad \hat{\mathbf{A}}=\left[-\hat{\mathbf{J}}_{\boldsymbol{\pi} \boldsymbol{\theta}} \hat{\mathbf{J}}_{\boldsymbol{\theta} \boldsymbol{\theta}}^{-1}: \quad \mathbf{I}_{m}\right]$, 
$\hat{\mathbf{\Omega}}_{\boldsymbol{\theta} \boldsymbol{\theta}}$ and $\hat{\mathbf{J}}_{\boldsymbol{\theta} \boldsymbol{\theta}}$ are given in Lemma 1 ,

$$
\begin{aligned}
& \hat{\mathbf{\Omega}}_{\pi \varphi}=\hat{v}_{c} \frac{\hat{\mathbf{R}}^{\prime} \hat{\mathbf{H}}^{-1 / 2} \hat{\mathbf{F}}}{T}+\frac{\left(\hat{k}_{c}-1\right)}{2} \frac{\hat{\mathbf{R}}^{\prime} \hat{\mathbf{C}}}{T}, \\
& \hat{\mathbf{\Omega}}_{\pi \eta}=\frac{\left(\hat{k}_{c}-1\right)}{2} \frac{\hat{\mathbf{R}}^{\prime} \hat{\mathbf{X}}}{T}, \\
& \hat{\mathbf{\Omega}}_{\pi \pi}=\left(\hat{k}_{c}-1\right) \frac{\hat{\mathbf{R}}^{\prime} \hat{\mathbf{R}}}{T}, \\
& \text { and } \hat{\mathbf{J}}_{\boldsymbol{\pi} \boldsymbol{\theta}}=\left[\hat{\mathbf{J}}_{\pi \varphi}: \hat{\mathbf{J}}_{\boldsymbol{\pi} \boldsymbol{\eta}}\right] \text { with } \\
& \hat{\mathbf{J}}_{\pi \varphi}=\frac{\hat{\mathbf{R}}^{\prime} \hat{\mathbf{C}}}{T}, \quad \hat{\mathbf{J}}_{\pi \eta}=\frac{\hat{\mathbf{R}}^{\prime} \hat{\mathbf{X}}}{T} .
\end{aligned}
$$

Observe that $\boldsymbol{\Sigma}=\mathbf{A} \boldsymbol{\Omega} \mathbf{A}^{\prime}$ depends upon the "mode" of estimation only through $\boldsymbol{\Omega}$ and not $\mathbf{J}_{\boldsymbol{\pi} \boldsymbol{\theta}}$, which is independent of the mode of estimation. In particular, and of relevance for later discussion, if $\mathbf{J}_{\pi \varphi}=\mathrm{E}\left[\mathbf{r}_{t}^{\infty} \mathbf{c}_{t}^{\infty \prime}\right]_{\boldsymbol{\theta}=\boldsymbol{\theta}_{0}}=\mathbf{0}$ then the limit distribution of $\sqrt{T} \mathbf{D}_{\boldsymbol{\pi} T}(\hat{\boldsymbol{\theta}})$ is not influenced by the estimation of $\boldsymbol{\varphi}$. Indeed, it appears that this claim, $\mathbf{J}_{\boldsymbol{\pi} \varphi}=\mathbf{0}$, is always made when constructing parametric misspecification tests of GARCH models under the assumption of conditional symmetry; see, e.g., Lundbergh and Teräsvirta (2002) and Engle and Ng (1993). Using the framework introduced here, it is argued in the next section that this is not the case, in general, and in particular it is not the case for the test procedures proposed by Lundbergh and Teräsvirta (2002) and Engle and Ng (1993).

Section 4 describes how (9) accommodates existing misspecification tests and also provides alternative asymptotically valid test procedures. Before that, however, the important effects of (known) conditional symmetry are considered, although normality of $\xi_{t}$ is not necessarily assumed.

\subsection{The Effects of Conditional Symmetry}

Conditional symmetry implies that $\mathrm{E}\left[\xi_{t}^{3}\right]=0, \mathrm{E}\left[\varepsilon_{0 t}^{3} \mid \mathcal{F}_{t-1}\right]=0$, and thus $v_{c}=0$. Although it can be tested (see e.g., Bai and Ng, 2001), it is often just assumed as in Lundbergh and Teräsvirta (2002) and Engle and Ng (1993), with the latter actually assuming normality of $\xi_{t}$. The presence of conditional symmetry simplifies the form $\boldsymbol{\Sigma}$ for the class of test indicators given by (9) as follows.

LEMMA 3. Under conditional symmetry

(i) $\mathbf{J}_{\eta \varphi}=\mathrm{E}\left[-\frac{\partial \mathbf{d}_{\eta t}^{\infty}\left(\boldsymbol{\theta}_{0}\right)}{\partial \boldsymbol{\varphi}^{\prime}}\right]=\mathbf{0}$;

(ii) $\boldsymbol{\Omega}_{\eta \varphi}=\mathrm{E}\left[\mathbf{d}_{\eta t}^{\infty}\left(\boldsymbol{\theta}_{0}\right) \mathbf{d}_{\varphi t}^{\infty}\left(\boldsymbol{\theta}_{0}\right)^{\prime}\right]=\mathbf{0}$;

(iii) $\boldsymbol{\Omega}_{\varphi \varphi}=\frac{\left(k_{c}-1\right)}{4} \mathrm{E}\left[\mathbf{c}_{t}^{\infty} \mathbf{c}_{t}^{\infty \prime}\right]_{\boldsymbol{\theta}=\boldsymbol{\theta}_{0}}+\mathrm{E}\left[\frac{1}{h_{t}^{\infty}} \mathbf{f}_{t} \mathbf{f}_{t}^{\prime}\right]_{\boldsymbol{\theta}=\boldsymbol{\theta}_{0}}$;

(iv) $\boldsymbol{\Omega}_{\boldsymbol{\pi} \varphi}=\frac{\left(k_{c}-1\right)}{2} \mathrm{E}\left[\mathbf{r}_{t}^{\infty} \mathbf{c}_{t}^{\infty \prime}\right]_{\boldsymbol{\theta}=\boldsymbol{\theta}_{0}}$. 
Some simple algebra reveals that, under conditional symmetry, if it is erroneously assumed that $\mathbf{J}_{\boldsymbol{\pi} \varphi}=\mathbf{0}$ in constructing the test statistic, the difference between the assumed, $\Sigma^{A}$, and true, $\boldsymbol{\Sigma}$, variance matrices is

$\Sigma^{A}-\Sigma=\mathbf{J}_{\pi \varphi} \mathbf{J}_{\varphi \varphi}^{-1}\left[\left(k_{c}-1\right) \mathbf{J}_{\varphi \varphi}-\mathbf{\Omega}_{\varphi \varphi}\right] \mathbf{J}_{\varphi \varphi}^{-1} \mathbf{J}_{\varphi \pi}$,

where $\left(k_{c}-1\right) \mathbf{J}_{\varphi \varphi}-\boldsymbol{\Omega}_{\varphi \varphi}=\left(\left(k_{c}-1\right) / 2\right) \mathrm{E}\left[\mathbf{c}_{t}^{\infty} \mathbf{c}_{t}^{\infty \prime}\right]+\left(k_{c}-2\right) \mathrm{E}\left[\left(1 / h_{t}^{\infty}\right) \mathbf{f}_{t} \mathbf{f}_{t}^{\prime}\right]$. Under normality, $k_{c}-1=2$, so that $\Sigma^{A}-\Sigma$ is positive definite when $\mathbf{J}_{\varphi \boldsymbol{\pi}}=\mathbf{J}_{\boldsymbol{\pi} \varphi}^{\prime}$ has full column rank and remains so under excess (normal) kurtosis. ${ }^{8}$ Because both $\Sigma^{A}$ and $\Sigma$ can be consistently estimated (see the discussion that follows), the procedure that utilizes the incorrect $\Sigma^{A}$ will be asymptotically undersized (under normality) and increasingly so under heavier tailed distributions.

3.1.1. Variance Matrix Estimators. Correspondingly, and given Lemmas 2 and 3, a consistent estimator for $\boldsymbol{\Sigma}$ can be obtained as

$$
\begin{aligned}
\hat{\boldsymbol{\Sigma}}_{T}=\frac{1}{T}[ & \frac{\hat{\vartheta}^{\prime} \hat{\vartheta}}{T} \hat{\mathbf{R}}^{\prime} \hat{\mathbf{M}}_{\mathbf{X}} \hat{\mathbf{R}}-\frac{\hat{\vartheta}^{\prime} \hat{\vartheta}}{T} \hat{\mathbf{R}}^{\prime} \hat{\mathbf{C}}\left(\hat{\mathbf{F}}^{\prime} \hat{\mathbf{H}}^{-1} \hat{\mathbf{F}}+\frac{1}{2} \hat{\mathbf{C}}^{\prime} \hat{\mathbf{C}}\right)^{-1} \hat{\mathbf{C}}^{\prime} \hat{\mathbf{R}} \\
& +\hat{\mathbf{R}}^{\prime} \hat{\mathbf{C}}\left(\hat{\mathbf{F}}^{\prime} \hat{\mathbf{H}}^{-1} \hat{\mathbf{F}}+\frac{1}{2} \hat{\mathbf{C}}^{\prime} \hat{\mathbf{C}}\right)^{-1}\left(\hat{\mathbf{F}}^{\prime} \hat{\mathbf{H}}^{-1} \hat{\mathbf{F}}+\frac{\hat{\vartheta}^{\prime} \hat{\vartheta}}{4 T} \hat{\mathbf{C}}^{\prime} \hat{\mathbf{C}}\right) \\
& \left.\times\left(\hat{\mathbf{F}}^{\prime} \hat{\mathbf{H}}^{-1} \hat{\mathbf{F}}+\frac{1}{2} \hat{\mathbf{C}}^{\prime} \hat{\mathbf{C}}\right)^{-1} \hat{\mathbf{C}}^{\prime} \hat{\mathbf{R}}\right]
\end{aligned}
$$

where, as before, "hats" denote evaluation at $\hat{\boldsymbol{\theta}}$ and $\mathbf{M}_{\mathbf{X}}=\mathbf{I}-\mathbf{X}\left(\mathbf{X}^{\prime} \mathbf{X}\right)^{-1} \mathbf{X}^{\prime}$. Further modifications can be made according to whether $\mathbf{J}_{\boldsymbol{\pi} \varphi}$ and/or $\mathbf{J}_{\boldsymbol{\pi} \boldsymbol{\eta}}$ are null matrices. The former case, $\mathbf{J}_{\boldsymbol{\pi} \varphi}=\mathbf{0}$, yields

$\hat{\boldsymbol{\Sigma}}_{1 T}=\frac{1}{T}\left[\frac{\hat{\boldsymbol{\vartheta}}^{\prime} \hat{\boldsymbol{\vartheta}}}{T} \hat{\mathbf{R}}^{\prime} \hat{\mathbf{M}}_{\mathbf{X}} \hat{\mathbf{R}}\right]$,

which is the form assumed by Lundbergh and Teräsvirta (2002) and Engle and $\mathrm{Ng}$ (1993), and the test statistic (12) has the simple interpretation as $T$ times the uncentered $R^{2}$ from regressing $\hat{\vartheta}$ on $[\hat{\mathbf{R}}, \hat{\mathbf{X}}]$. The latter case, $\mathbf{J}_{\pi \eta}=\mathbf{0}$, yields

$$
\begin{aligned}
\hat{\boldsymbol{\Sigma}}_{2 T}=\frac{1}{T}[ & \frac{\hat{\boldsymbol{\vartheta}}^{\prime} \hat{\boldsymbol{\vartheta}}}{T} \hat{\mathbf{R}}^{\prime} \hat{\mathbf{R}}-\frac{\hat{\boldsymbol{\vartheta}}^{\prime} \hat{\boldsymbol{\vartheta}}}{T} \hat{\mathbf{R}}^{\prime} \hat{\mathbf{C}}\left(\hat{\mathbf{F}}^{\prime} \hat{\mathbf{H}}^{-1} \hat{\mathbf{F}}+\frac{1}{2} \hat{\mathbf{C}}^{\prime} \hat{\mathbf{C}}\right)^{-1} \hat{\mathbf{C}}^{\prime} \hat{\mathbf{R}} \\
& +\hat{\mathbf{R}}^{\prime} \hat{\mathbf{C}}\left(\hat{\mathbf{F}}^{\prime} \hat{\mathbf{H}}^{-1} \hat{\mathbf{F}}+\frac{1}{2} \hat{\mathbf{C}}^{\prime} \hat{\mathbf{C}}\right)^{-1}\left(\hat{\mathbf{F}}^{\prime} \hat{\mathbf{H}}^{-1} \hat{\mathbf{F}}+\frac{\hat{\vartheta}^{\prime} \hat{\vartheta}}{4 T} \hat{\mathbf{C}}^{\prime} \hat{\mathbf{C}}\right) \\
& \left.\times\left(\hat{\mathbf{F}}^{\prime} \hat{\mathbf{H}}^{-1} \hat{\mathbf{F}}+\frac{1}{2} \hat{\mathbf{C}}^{\prime} \hat{\mathbf{C}}\right)^{-1} \hat{\mathbf{C}}^{\prime} \hat{\mathbf{R}}\right]
\end{aligned}
$$


If both $\mathbf{J}_{\boldsymbol{\pi} \varphi}$ and $\mathbf{J}_{\boldsymbol{\pi} \boldsymbol{\eta}}$ are null matrices, we obtain

$\hat{\mathbf{\Sigma}}_{3 T}=\frac{1}{T}\left[\frac{\hat{\vartheta}^{\prime} \hat{\vartheta}}{T} \hat{\mathbf{R}}^{\prime} \hat{\mathbf{R}}\right]$

and the test statistic (12) becomes $T$ times the uncentered $R^{2}$ from regressing $\hat{\vartheta}$ on $\hat{\mathbf{R}}$.

3.1.2. Orthogonality. Importantly, Lemma 3 shows that $\hat{\boldsymbol{\varphi}}$ and $\hat{\boldsymbol{\eta}}$ are asymptotically orthogonal within a QML framework. ${ }^{9}$ Thus, consistent estimation of $\boldsymbol{\eta}_{0}$ can be achieved by exploiting the QML approach to obtain $\hat{\eta}$ but utilizing any $\sqrt{T}$-consistent estimator, $\hat{\boldsymbol{\varphi}}$ (see Cox and Reid, 1987), without loss of asymptotic efficiency in estimating $\boldsymbol{\eta}_{0}$, although there will be a loss of efficiency in small samples; e.g., the least squares estimator of $\boldsymbol{\varphi}$ could be employed. This might suggest that tests for the adequacy of $h_{t}$ will not be influenced (asymptotically, at least) by the estimation of $\boldsymbol{\varphi}$. Although this intuition is correct, e.g., when constructing tests for unconditional heteroskedasticity in the linear model, it is flawed when applied to certain misspecification tests for GARCH models (in particular, asymmetry and nonlinearity tests). Formally, as the proof of Lemma 2 makes clear, what is required is that $\mathbf{J}_{\boldsymbol{\pi} \varphi}=\mathbf{0}$, and although this appears to have been taken for granted by many authors the following example illustrates, quite nicely, that it should not be. The example employs an ARCH model that is technically not nested in the class of models characterized by Assumption A. However, assumptions such as those in Weiss (1986) could be exploited to get the same form of limit distribution as described in Theorem 2, with the obvious redefinitions of $\mathbf{x}_{t}$ and $\mathbf{c}_{t}$.

\section{Example 1}

Suppose we have the following model:

$$
\begin{aligned}
y_{t} & =\varphi+\varepsilon_{t}, \\
h_{t}^{a} & =1+\alpha_{1} \varepsilon_{t-1}^{2}+\pi \varepsilon_{t-1}, \quad 0<\alpha_{1}<1, \quad \pi>0
\end{aligned}
$$

and we want to test the null hypothesis that $\pi=0$, such that the null model for the conditional variance is

$h_{t}=1+\alpha_{1} \varepsilon_{t-1}^{2}, \quad 0<\alpha_{1}<1$

and the test indicator in (9) is $r_{t}=\varepsilon_{t-1} /\left(1+\alpha_{1} \varepsilon_{t-1}^{2}\right) \equiv r_{t}^{\infty}$, so that $\operatorname{var}\left(\varepsilon_{t}\right)=1 /\left(1-\alpha_{1}\right)$ and $c_{t}=\frac{1}{h_{t}} \frac{\partial h_{t}}{\partial \varphi}=-2\left(\alpha_{1} \varepsilon_{t-1}\right) /\left(1+\alpha_{1} \varepsilon_{t-1}^{2}\right) \equiv c_{t}^{\infty}$. We assume $\mathrm{E}\left[\varepsilon_{0 t}^{3} \mid \mathcal{F}_{t-1}\right]=0$, so that Lemma 3(i) implies that $J_{\eta \varphi}=0$, a scalar, and thus $\hat{\varphi}$ and $\hat{\eta}=\hat{\alpha}_{1}$ are asymptotically orthogonal. However, in this case, the scalar $J_{\pi \varphi}=\mathrm{E}\left[r_{t} c_{t}\right]_{\theta=\theta_{0}}$ is given by 
$-2 E\left\{\mathrm{E}\left[\frac{\alpha_{1} \varepsilon_{t-1}^{2}}{\left(1+\alpha_{1} \varepsilon_{t-1}^{2}\right)^{2}} \mid \mathcal{F}_{t-2}\right]\right\}_{\theta=\theta_{0}}=-2 \mathrm{E}\left[\frac{\alpha_{1} \varepsilon_{t-1}^{2}}{\left(1+\alpha_{1} \varepsilon_{t-1}^{2}\right)^{2}}\right]_{\theta=\theta_{0}}$.

Then, assuming $\operatorname{Pr}\left(\varepsilon_{t-1} \neq 0\right)>0$, it follows that $\frac{\alpha_{1} \varepsilon_{t-1}^{2}}{\left(1+\alpha_{1} \varepsilon_{t-1}^{2}\right)^{2}}>0$, almost everywhere. Moreover, $\operatorname{Pr}\left(\frac{\alpha_{1} \varepsilon_{t-1}^{2}}{\left(1+\alpha_{1} \varepsilon_{t-1}^{2}\right)^{2}}<1\right)=1$. Therefore $\operatorname{Pr}\left(0<\frac{\alpha_{1} \varepsilon_{t-1}^{2}}{\left(1+\alpha_{1} \varepsilon_{t-1}^{2}\right)^{2}}<1\right)=1$, so that $J_{\pi \varphi}$ exists and is bounded between -2 and 0 .

This example is of relevance because it is a special case of the nonlinearity test proposed by Lundbergh and Teräsvirta (2002). In the construction of that test statistic, QML is employed, and it is explicitly "stated" that, because of symmetry, $\mathrm{E}\left[T \mathbf{D}_{\boldsymbol{\pi} T}\left(\boldsymbol{\theta}_{0}\right) \mathbf{D}_{\boldsymbol{\theta} T}\left(\boldsymbol{\theta}_{0}\right)^{\prime}\right]=\mathbf{0}$ (Lundbergh and Teräsvirta, 2002, p. 433). From this the researchers incorrectly assume that there are negligible estimation effects, i.e., that $\mathbf{J}_{\boldsymbol{\pi} \varphi}=\mathrm{E}\left[\mathbf{r}_{t}^{\infty} \mathbf{c}_{t}^{\infty \prime}\right]_{\boldsymbol{\theta}=\boldsymbol{\theta}_{0}}=\mathbf{0}$. However, the preceding simple example illustrates that this is not true. Generalizing this to the GARCH model, Section 4 shows that for the tests proposed by Lundbergh and Teräsvirta (2002) and Engle and $\mathrm{Ng}$ (1993) $\mathbf{J}_{\boldsymbol{\pi} \varphi}$ is nonzero, rendering these test procedures asymptotically invalid even under conditional symmetry.

\section{TESTING FOR NONLINEARITY AND ASYMMETRY}

In this section, we illustrate the usefulness of the general framework described in Section 3 in two ways. First in Section 4.1, the general asymptotic analysis is applied to the Lundbergh and Teräsvirta (2002) test for nonlinearity and the Engle and $\mathrm{Ng}$ (1993) negative size bias test for asymmetry. It is shown that both are asymptotically invalid procedures, even if the conditional distribution of $\xi_{t}$ is symmetric. Second the framework of Section 3 justifies two alternative, and asymptotically valid, tests for nonlinearity and asymmetry in the conditional variance $h_{t}$. All of the ensuing analysis is undertaken under the assumption of conditional symmetry of the errors so that Lemma 3 applies, and a consistent estimator for $\boldsymbol{\Sigma}$, in the limit distribution of $\sqrt{T} \mathbf{D}_{\boldsymbol{\pi} T}(\hat{\boldsymbol{\theta}})$ given in Theorem 2, is discussed in Section 3.1.1. ${ }^{10}$

\subsection{An Analysis of Existing Tests}

4.1.1. Lundbergh and Teräsvirta Test. To test against nonlinearity in the GARCH specification, Lundbergh and Teräsvirta (2002, Thm. 4.1) proposed the following statistic:

$$
T_{L T}=T \times \frac{\hat{\vartheta}^{\prime} \hat{\mathbf{G}}\left(\hat{\mathbf{G}}^{\prime} \hat{\mathbf{G}}\right)^{-1} \hat{\mathbf{G}}^{\prime} \hat{\vartheta}}{\hat{\vartheta}^{\prime} \hat{\vartheta}},
$$


where $\hat{\mathbf{G}}$ is a matrix with rows $\hat{\mathbf{g}}_{t}^{\prime}=\left(\hat{\mathbf{x}}_{t}^{\prime}, \hat{\mathbf{v}}_{t-1}^{\prime}\right)$ and $\hat{\mathbf{v}}_{t-1}=\left(\hat{\mathbf{v}}_{1, t-1}^{\prime}, \hat{\mathbf{v}}_{3, t-1}^{\prime}, \ldots\right.$, $\left.\hat{\mathbf{v}}_{n+2, t-1}^{\prime}\right)^{\prime}$, with $\hat{\mathbf{v}}_{s, t-1}=\left(\hat{\varepsilon}_{t-1}^{s}, \hat{\varepsilon}_{t-2}^{s}, \ldots, \hat{\varepsilon}_{t-q}^{s}\right)^{\prime}$. This can be interpreted as $T$ times the uncentered $R^{2}$ following a regression of $\hat{\vartheta}$ on $\hat{\mathbf{G}}$ and is assumed to be asymptotically distributed as a $\chi_{(n+1) q}^{2}$ random variable under the null. In terms of the general framework of Section 3, the test indicator is of the form (9), with test variables $\hat{\mathbf{r}}_{t}=\hat{\mathbf{v}}_{t-1} \cdot{ }^{11}$ Lundbergh and Teräsvirta (2002) also advocated an alternative regression-based procedure, following Wooldridge (1991), which they suggested is robust to nonnormality. However, the modification employed is actually designed to make the statistic robust to heterokurticity (as Wooldridge, 1991, p. 29, makes clear), not nonnormality. But heterokurticity is ruled out anyway by the assumptions made on $\xi_{t}$, and so this alternative form is not considered further.

To focus discussion, consider a null $\operatorname{GARCH}(1,1)$ model with $n=1$, so that $\hat{\mathbf{r}}_{t}=\left(\hat{\varepsilon}_{t-1}, \hat{\varepsilon}_{t-1}^{3}\right)^{\prime}$. The following lemma generalizes the example of the previous section and establishes that $\mathbf{J}_{\boldsymbol{\pi} \varphi} \neq \mathbf{0}$ whereas $\mathbf{J}_{\boldsymbol{\pi} \boldsymbol{\eta}}=\mathbf{0}$. The former result implies that the test procedure proposed by Lundbergh and Teräsvirta (2002) is asymptotically invalid. ${ }^{12}$

LEMMA 4. Assuming the GARCH(1,1) model under the null hypothesis and the test variables considered by Lundbergh and Teräsvirta (2002) of $\hat{\mathbf{r}}_{t}=\left(\hat{\varepsilon}_{t-1}\right.$, $\left.\hat{\varepsilon}_{t-1}^{3}\right)^{\prime},(10)$ becomes

$$
\mathbf{J}_{\boldsymbol{\pi} \varphi}=-2 \alpha_{01} \mathrm{E}\left[\frac{1}{h_{t}^{\infty}}\left(\begin{array}{c}
\varepsilon_{t-1} \\
\varepsilon_{t-1}^{3}
\end{array}\right) \sum_{i=0}^{\infty} \beta_{1}^{i} \varepsilon_{t-1-i} \mathbf{f}_{t-1-i}^{\prime}\right]_{\boldsymbol{\theta}=\boldsymbol{\theta}_{0}}
$$

$\neq \mathbf{0}$,

in general, and (11) becomes

$\mathbf{J}_{\pi \eta}=\mathrm{E}\left[\frac{1}{h_{t}^{\infty}}\left(\begin{array}{c}\varepsilon_{t-1} \\ \varepsilon_{t-1}^{3}\end{array}\right) \sum_{i=0}^{\infty} \beta_{1}^{i} \mathbf{s}_{t-1-i}^{\infty \prime}\right]_{\boldsymbol{\theta}=\boldsymbol{\theta}_{0}}=\mathbf{0}$.

The implication of this is that, rather than employing the variance estimator $\hat{\boldsymbol{\Sigma}}_{1 T}$, given in equation (14), Lundbergh and Teräsvirta (2002) should have employed version $\hat{\boldsymbol{\Sigma}}_{2 T}$, given in equation (15), or an asymptotically equivalent version thereof.

4.1.2. Engle and Ng Test. Among the most popular asymmetry tests are those proposed by Engle and $\mathrm{Ng}$ (1993). To confirm the asymmetric behavior of financial series, they constructed a number of score type tests. For purposes of exposition, consider the negative size bias test that examines the significance of (9), employing the (scalar) test variable $\hat{r}_{t}=\hat{I}_{t-1} \hat{\varepsilon}_{t-1}$ where the indicator function $I_{t-1}$ takes the value one if $\varepsilon_{t-1} \leq 0$ and zero otherwise. 
Specifically, the test statistic proposed by Engle and $\mathrm{Ng}$ (1993) is constructed as follows:

$T_{E N}=T \times \frac{\hat{\vartheta}^{\prime} \hat{\mathbf{G}}\left(\hat{\mathbf{G}}^{\prime} \hat{\mathbf{G}}\right)^{-1} \hat{\mathbf{G}}^{\prime} \hat{\vartheta}}{\hat{\vartheta}^{\prime} \hat{\vartheta}}$,

where here $\hat{\mathbf{G}}$ has rows $\mathbf{g}_{t}^{\prime}=\left(\hat{\mathbf{x}}_{t}^{\prime}, \hat{I}_{t-1} \hat{\varepsilon}_{t-1}\right)$ and $T_{E N}$ is assumed to be asymptotically distributed as $\chi_{1}^{2}$ under the null. This can be computed as $T$ times the uncentered $R^{2}$ following a regression of $\hat{\vartheta}$ on $\hat{\mathbf{G}}$. The tests presented in their paper are derived assuming a conditional normal distribution for $\xi_{t}$, although asymptotically valid procedures can be derived assuming just conditional symmetry, as is done here.

This case is not consistent with the assumption that $\mathbf{D}_{\boldsymbol{\pi} T}(\hat{\boldsymbol{\theta}})$ is continuously differentiable, as required for the analysis of Section 3. A direct mean value expansion of $\sqrt{T} \mathbf{D}_{\boldsymbol{\pi} T}(\hat{\boldsymbol{\theta}})$ is not applicable because it entails terms such as $\partial \mathbf{r}_{t} / \partial \boldsymbol{\theta}^{\prime}$, and this issue was not discussed by Engle and $\mathrm{Ng}$ (1993). Therefore, in general (and to deal with such a possibility), it will be assumed that

$$
\sqrt{T} \mathbf{D}_{\pi T}(\hat{\boldsymbol{\theta}})=\frac{1}{\sqrt{T}} \sum_{t=1}^{T}\left[\left(\frac{\hat{\varepsilon}_{t}^{2}}{\hat{h}_{t}}-1\right) \mathbf{r}_{t}\left(\boldsymbol{\theta}_{0}\right)\right]+o_{p}(1) .
$$

(Note that employing (19) does not alter the generic expressions for $\mathbf{J}_{\boldsymbol{\pi} \varphi}$ and $\mathbf{J}_{\boldsymbol{\pi} \boldsymbol{\eta}}$ given by (10) and (11), respectively.) This assumption is innocuous when $\mathbf{r}_{t}(\boldsymbol{\theta})$ is continuously differentiable in $\boldsymbol{\theta}$, because then $\mathbf{r}_{t}(\hat{\boldsymbol{\theta}})=r_{t}\left(\boldsymbol{\theta}_{0}\right)+\left(\partial \mathbf{r}_{t}(\overline{\boldsymbol{\theta}}) / \partial \boldsymbol{\theta}\right)\left(\hat{\boldsymbol{\theta}}-\boldsymbol{\theta}_{0}\right)$ and $\left(\hat{\boldsymbol{\theta}}-\boldsymbol{\theta}_{0}\right)$ is $O_{p}\left(T^{-1 / 2}\right)$. When $\mathbf{r}_{t}$ is not continuously differentiable, (19) will have to be verified on a case by case basis, and the following result verifies this for the negative size bias test procedure.

PROPOSITION 1. For the negative size bias test of Engle and $\mathrm{Ng}$ (1993), in which $r_{t}=I_{t-1} \varepsilon_{t-1}$ is not continuously differentiable in $\boldsymbol{\theta}$, the equality in equation (19) holds.

Again it is found that the Engle and $\mathrm{Ng}$ (1993) tests are asymptotically invalid, in general, under the null hypothesis because (18) assumes that $\mathbf{J}_{\pi \varphi}=\mathbf{0}$, contrary to the following lemma.

LEMMA 5. Assuming the GARCH(1,1) model under the null hypothesis and test variable $\hat{r}_{t}=\hat{I}_{t-1} \hat{\varepsilon}_{t-1},(10)$ becomes

$\mathbf{J}_{\pi \boldsymbol{\varphi}}=-2 \alpha_{01} \mathrm{E}\left[\frac{1}{h_{t}^{\infty}} I_{t-1} \varepsilon_{t-1} \sum_{i=0}^{\infty} \beta_{1}^{i} \varepsilon_{t-1-i} \mathbf{f}_{t-1-i}^{\prime}\right]_{\boldsymbol{\theta}=\boldsymbol{\theta}_{0}} \neq \mathbf{0}$,

in general. 


\subsection{Alternative Tests}

Section 4.1 detailed the asymptotic invalidity of tests proposed by both Lundbergh and Teräsvirta (2002) and Engle and $\mathrm{Ng}$ (1993). Of course, asymptotically valid test procedures can be obtained using the framework of Section 3, together with the test variables employed by these authors.

However, these test variables are derived from a particular alternative specification for the conditional heteroskedasticity. Specifically, the alternative model employed by Lundbergh and Teräsvirta (2002, p. 422) is

$\varepsilon_{t}=\varsigma_{t} \sqrt{h_{t}+g_{t}}$,

where $\varsigma_{t}$ are i.i.d. (zero mean and unit variance) random variables, whereas that proposed by Engle and $\mathrm{Ng}$ (1993, p. 1758) is of the form

$\varepsilon_{t}=\varsigma_{t} \sqrt{h_{t} \exp \left(g_{t}\right)}$

in which $h_{t}=\boldsymbol{\eta}^{\prime} \mathbf{s}_{t-1}$ and $g_{t}=g\left(\boldsymbol{\pi} ; \mathbf{v}_{t-1}\right)$ characterizes the misspecification where $\mathbf{v}_{t-1}$ is the vector of omitted variables. In particular, the nonlinearity test of Lundbergh and Teräsvirta (2002) is constructed from the following alternative:

$h_{t}^{a}=\alpha_{0}+\sum_{j=1}^{q} \alpha_{j} \varepsilon_{t-j}^{2}+g\left(\boldsymbol{\pi} ; \mathbf{v}_{t-1}\right)+\sum_{i=1}^{p} \beta_{i} h_{t-i}$,

whereas that of Engle and $\mathrm{Ng}(1993)$ is

$\ln \left(h_{t}^{a}\right)=\ln \left(\alpha_{0}+\sum_{j=1}^{q} \alpha_{j} \varepsilon_{t-j}^{2}+\sum_{i=1}^{p} \beta_{i} h_{t-i}\right)+g\left(\boldsymbol{\pi} ; \mathbf{v}_{t-1}\right)$.

Within the QML approach, which uses (5), the tests actually constructed by Lundbergh and Teräsvirta (2002) and Engle and $\mathrm{Ng}$ (1993) can be interpreted as score tests of $h_{t}$ against the alternatives of (20) and (21), respectively, i.e., tests of $H_{0}: \boldsymbol{\pi}=\mathbf{0}$. Although this yields asymptotically valid (quasi-score) test procedures using the framework of Section 3, the alternative models proposed in the literature, and considered by Lundbergh and Teräsvirta (2002) and Engle and Ng (1993) in their Monte Carlo studies, are not of the form (20) or (21). In those studies, the power of the test is evaluated against alternative models for the conditional heteroskedasticity (specifically GJR-GARCH and EGARCH models) that are "recursive" in nature. This characteristic is not apparent in (20) or (21), where $h_{t-i}, i=1, \ldots, p$, appears on the right-hand side and not the lagged values of $h_{t}^{a}$. For example, the GJR-GARCH $(1,1)$ model can be expressed in the following form:

$h_{t}^{a}=\alpha_{0}+\alpha_{1} \varepsilon_{t-1}^{2}+\alpha_{2} I_{t-1} \varepsilon_{t-1}^{2}+\beta_{1} h_{t-1}^{a}$,

indicating that the conditional heteroskedasticity is "recursive" in nature as a result of the inclusion of $h_{t-1}^{a}$ on the right-hand side. As a consequence, the 
nonlinearity/asymmetry tests, which neglect this recursive behavior under the alternative, may well lack power against these specifications. Similar remarks apply for the parameter constancy test constructed by Lundbergh and Teräsvirta (2002).

With this in mind, alternative tests for nonlinearity and asymmetry are now constructed with the following alternative specification in mind:

$$
\begin{aligned}
\varepsilon_{t} & =\varsigma_{t}\left(h_{t}^{a}\right)^{1 / 2}, \\
h_{t}^{a} & =\boldsymbol{\eta}^{\prime} \mathbf{s}_{t-1}^{a}+g_{t}=\left(a_{t}+g_{t}\right)+B(L) h_{t}^{a},
\end{aligned}
$$

where $\mathbf{s}_{t-1}^{a}=\left(1, \varepsilon_{t-1}^{2}, \ldots, \varepsilon_{t-q}^{2}, h_{t-1}^{a}, \ldots, h_{t-p}^{a}\right)^{\prime}$ and $g_{t}=g\left(\mathbf{v}_{t-1} ; \boldsymbol{\pi}\right)$ is a nonlinear and/or asymmetric function of $\varepsilon_{t-j}, j \geq 1$ with $\mathbf{v}_{t-1}$ being the vector of omitted variables. Thus the test indicator is of the form (9), with test variables constructed as $\hat{\mathbf{r}}_{t}=\left[\frac{1}{h_{t}^{a}} \frac{\partial h_{t}^{a}}{\partial \boldsymbol{\pi}}\right]_{\boldsymbol{\pi}=\mathbf{0}, \boldsymbol{\theta}=\hat{\boldsymbol{\theta}}}$.

4.2.1. Testing for Nonlinearity. Following Lundbergh and Teräsvirta (2002), nonlinearity is introduced in the intercept and the term containing the squared past errors via a smooth transition function $F_{n}\left(\varepsilon_{t-j} ; \gamma, \mathbf{c}\right), j=1, \ldots, q$, i.e.,

$g_{t}=\sum_{j=1}^{q}\left(\alpha_{0 j}+\alpha_{1 j} \varepsilon_{t-j}^{2}\right) F_{n}\left(\varepsilon_{t-j} ; \gamma, \mathbf{c}\right)$

with

$$
\begin{gathered}
F_{n}\left(\varepsilon_{t-j} ; \gamma, \mathbf{c}\right)=\left(1+\exp \left(-\gamma \prod_{l=1}^{n}\left(\varepsilon_{t-j}-c_{l}\right)\right)\right)^{-1}-\frac{1}{2}, \\
\gamma>0, \quad c_{1} \leq \cdots \leq c_{n} .
\end{gathered}
$$

For example, if the location parameter (threshold) of the transition function is zero, i.e., $\mathbf{c}=\mathbf{0}$, then the transition is made between the regime characterized by negative shocks to the one characterized by positive shocks. Under the null of $\gamma=0$, it follows that $F_{n}=0$, and taking a first-order Taylor expansion of $F_{n}$ around $\gamma=0$ yields

$g_{t}=\boldsymbol{\pi}^{\prime} \mathbf{v}_{t-1}$

where $\mathbf{v}_{t-1}=\left(\mathbf{v}_{1, t-1}^{\prime}, \mathbf{v}_{3, t-1}^{\prime}, \ldots, \mathbf{v}_{n+2, t-1}^{\prime}\right)$, with $\mathbf{v}_{s, t-1}=\left(\varepsilon_{t-1}^{s}, \varepsilon_{t-2}^{s}, \ldots, \varepsilon_{t-q}^{s}\right)^{\prime}$, $s=1,3, \ldots, n+2$.

Combining (22) and (24), a quasi-score test of $\boldsymbol{\pi}=\mathbf{0}$ can be based on assessing the significance of the test indicator (9) in which the test variables, given $\tilde{\boldsymbol{\varepsilon}}_{0}$, are constructed as 


$$
\begin{aligned}
\hat{\mathbf{r}}_{t} & =\left[\frac{1}{h_{t}^{a}} \frac{\partial h_{t}^{a}}{\partial \boldsymbol{\pi}}\right]_{\boldsymbol{\pi}=\mathbf{0}, \boldsymbol{\theta}=\hat{\boldsymbol{\theta}}} \\
& =\frac{1}{\hat{h}_{t}} \sum_{i=0}^{t-1} \hat{\psi}_{i} \hat{\mathbf{v}}_{t-1-i}^{\prime} \\
& =\frac{1}{\hat{h}_{t}} \sum_{i=0}^{t-1}\left\{\hat{B}^{*}(L)\right\}^{i} \hat{\mathbf{v}}_{t-1-i}^{\prime},
\end{aligned}
$$

where $\hat{B}^{*}(L)=\hat{\beta}_{1}+\hat{\beta}_{2} L+\cdots+\hat{\beta}_{p} L^{p-1}$ and, in practice, $\frac{\partial h_{t}^{a}}{\partial \pi}$ can be derived from the recursion $\frac{\partial h_{t}^{a}}{\partial \boldsymbol{\pi}}=\mathbf{v}_{t-1}+B(L) \frac{\partial h_{t}^{a}}{\partial \boldsymbol{\pi}}$. For example, in the $\operatorname{GARCH}(1,1)$ model assuming $n=1$, the test variables take the form

$\hat{\mathbf{r}}_{t}=\frac{1}{\hat{h}_{t}} \sum_{i=0}^{t-1} \hat{\beta}_{1}^{i}\left(\begin{array}{l}\hat{\varepsilon}_{t-1-i} \\ \hat{\varepsilon}_{t-1-i}^{3}\end{array}\right)$

compared with those employed by Lundbergh and Teräsvirta (2002), which are simply $\hat{\mathbf{r}}_{t}=\left(\hat{\varepsilon}_{t-1}, \hat{\varepsilon}_{t-1}^{3}\right)^{\prime}$ in this case.

The following lemma, stated for the general $\operatorname{GARCH}(p, q)$ model, establishes that $\mathbf{J}_{\pi \varphi}$ cannot be guaranteed to be zero even under conditional symmetry, although it turns out that $\mathbf{J}_{\pi \eta}=\mathbf{0}$ (so that $\boldsymbol{\Omega}_{\pi \eta}=\mathbf{0}$ also).

LEMMA 6. Under the null GARCH $(p, q)$ model with test variables given by $\hat{\mathbf{r}}_{t}=\frac{1}{\hat{h}_{t}} \sum_{i=0}^{t-1} \hat{\psi}_{i} \hat{\mathbf{v}}_{t-1-i}^{\prime}$, and (for simplicity, but without loss of generality) $n=1$, so that $\mathbf{v}_{t-1}=\left(\varepsilon_{t-1}, \varepsilon_{t-2}, \ldots, \varepsilon_{t-q}, \varepsilon_{t-1}^{3}, \varepsilon_{t-2}^{3}, \ldots, \varepsilon_{t-q}^{3}\right)^{\prime}$,

$\mathbf{J}_{\pi \varphi}=-2 \sum_{k=1}^{q} \alpha_{0 k} \mathrm{E}\left[\frac{1}{\left(h_{t}^{\infty}\right)^{2}} \sum_{i=0}^{\infty} \sum_{j=0}^{\infty} \psi_{i} \psi_{j} \varepsilon_{t-k-j} \mathbf{v}_{t-1-i} \mathbf{f}_{t-k-j}^{\prime}\right]_{\boldsymbol{\theta}=\boldsymbol{\theta}_{0}} \neq \mathbf{0}$

in general, but

$\mathbf{J}_{\boldsymbol{\pi} \boldsymbol{\eta}}=\mathrm{E}\left[\frac{1}{\left(h_{t}^{\infty}\right)^{2}} \sum_{i=0}^{\infty} \sum_{j=0}^{\infty} \psi_{i} \psi_{j} \mathbf{v}_{t-1-i} \mathbf{s}_{t-1-j}^{\infty \prime}\right]_{\boldsymbol{\theta}=\boldsymbol{\theta}_{0}}=\mathbf{0}$

Using these results and those of Section 3.1.1, an asymptotically valid nonlinearity test statistic can be constructed as

$$
T_{N}=T \mathbf{D}_{\pi T}(\hat{\boldsymbol{\theta}})^{\prime} \hat{\boldsymbol{\Sigma}}_{2 T}^{-1} \mathbf{D}_{\boldsymbol{\pi} T}(\hat{\boldsymbol{\theta}})
$$

which is asymptotically distributed as $\chi_{(n+1) q}^{2}$ under the null, for the general $n$ case, where $\operatorname{dim}\left(\mathbf{v}_{t-1}\right)=(n+1) q$ and $\hat{\boldsymbol{\Sigma}}_{2 T}$ is given by (15). 
4.2.2. Testing for Asymmetry. The asymmetry test, of whether important negative shocks have more impact on volatility than important positive shocks, assesses whether the variables $\mathbf{v}_{t-1}=\left(I_{t-1} \varepsilon_{t-1}, \ldots, I_{t-q} \varepsilon_{t-q}\right)^{\prime}$ have been omitted from the null $\operatorname{GARCH}(p, q)$ model. Again a quasi-score test statistic is constructed from the "alternative" volatility model of (22), with asymmetry characterized by $g_{t}=\boldsymbol{\pi}^{\prime} \mathbf{v}_{t-1}$. Within this framework and under the null of $\boldsymbol{\pi}=\mathbf{0}$, the test indicator in (9) employs test variables

$$
\begin{aligned}
\hat{\mathbf{r}}_{t} & =\frac{1}{\hat{h}_{t}} \sum_{i=0}^{t-1} \hat{\psi}_{i} \hat{\mathbf{v}}_{t-1-i} \\
& =\frac{1}{\hat{h}_{t}} \sum_{i=0}^{t-1}\left\{\hat{B}^{*}(L)\right\}^{i} \hat{\mathbf{v}}_{t-1-i} .
\end{aligned}
$$

If the null model is the $\operatorname{GARCH}(1,1)$ specification, the (scalar) test variable is

$\hat{r}_{t}=\frac{1}{\hat{h}_{t}} \sum_{i=0}^{t-1} \hat{\beta}_{1}^{i} \hat{I}_{t-1-i} \hat{\varepsilon}_{t-1-i}$

(This test variable differs from the Engle and $\mathrm{Ng}, 1993$, test variable of $\hat{r}_{t}=$ $\hat{I}_{t-1} \hat{\varepsilon}_{t-1}$ in this case.)

For this test indicator, neither $\mathbf{J}_{\pi \varphi}$ nor $\mathbf{J}_{\pi \eta}$ is a null matrix, in general, as stated by the following lemma.

LEMMA 7. Under the null GARCH $(p, q)$ model, with test variables given by $\hat{\mathbf{r}}_{t}=\frac{1}{\hat{h}_{t}} \sum_{i=0}^{t-1} \hat{\psi}_{i} \hat{\mathbf{v}}_{t-1-i}, \mathbf{v}_{t-1}=\left(I_{t-1} \varepsilon_{t-1}, \ldots, I_{t-q} \varepsilon_{t-q}\right)^{\prime}, \mathbf{J}_{\boldsymbol{\pi} \varphi} \neq \mathbf{0}$ and $\mathbf{J}_{\boldsymbol{\pi} \eta} \neq \mathbf{0}$, in general.

The discussion in Section 3.1 provides the following test statistic:

$T_{A}=T \mathbf{D}_{\pi T}(\hat{\boldsymbol{\theta}})^{\prime} \hat{\boldsymbol{\Sigma}}_{T}^{-1} \mathbf{D}_{\boldsymbol{\pi} T}(\hat{\boldsymbol{\Sigma}})$,

where $\hat{\Sigma}_{T}$ is given by (13) and $T_{A}$ is asymptotically distributed as $\chi_{q}^{2}$.

As argued by Engle and $\mathrm{Ng}$ (1993), we can also test asymmetry for more extreme values of past errors. The asymptotic distribution of the test in this case is the same as the previous one except that the test indicator employs variables $\hat{\mathbf{r}}_{t}=\hat{h}_{t}^{-1} \sum_{i=0}^{t-1}\left\{\hat{B}^{*}(L)\right\}^{i}\left(\hat{I}_{t-1} \hat{\varepsilon}_{t-1}^{2}, \ldots, \hat{I}_{t-q} \hat{\varepsilon}_{t-q}^{2}\right)^{\prime}$.

\section{MONTE CARLO STUDY}

In this section, Monte Carlo evidence is presented on the finite-sample size and power performance of the various asymmetry and nonlinearity tests discussed in Section 4. 
The Monte Carlo experiment for assessing the size properties of the tests is based on an $\operatorname{AR}(1)-G A R C H(1,1)$ data generation process. We consider the following four sets of parameter values for the conditional mean:

$\mathrm{M} 1: y_{t}=\varepsilon_{t}$,

M2-M4: $y_{t}=\varphi_{0}+\varphi_{1} y_{t-1}+\varepsilon_{t} \quad$ with $\varphi_{0}=1 \quad$ and $\quad \varphi_{1} \in\{0.1,0.5,0.9\}$,

where $\varepsilon_{t}=\sqrt{h_{t}} \xi_{t}$ with $\xi_{t} \sim N(0,1)$, or $\xi_{t} \sim t(v)$ (standardized Student $t$ distribution with $v$ degrees of freedom) with $v \in\{7,5,3\}$. The inclusion of $t(3)$, e.g., offers some evidence on the robustness of the procedures to violations of the moment assumptions employed. The conditional variance equation follows Engle and $\mathrm{Ng}$ (1993), taking one of the following forms:

$\mathrm{H}$ (High persistence) $: h_{t}=0.01+0.09 \varepsilon_{t-1}^{2}+0.9 h_{t-1}$,

$\mathrm{M}$ (Medium persistence) $: h_{t}=0.05+0.05 \varepsilon_{t-1}^{2}+0.9 h_{t-1}$,

L (Low persistence) $: h_{t}=0.2+0.05 \varepsilon_{t-1}^{2}+0.75 h_{t-1}$,

such that, without loss of generality, the unconditional variance of $\varepsilon_{t}$ equals one.

Combining the conditional mean and variance specifications yields 12 models to consider. For this purpose, a series of 1,200 data realizations was generated using the random generator number in GAUSS 5.0, with the first 200 observations being discarded to avoid initialization effects, yielding a sample size of 1,000 observations. Each model is replicated and estimated 1,000 times by QML. The test statistics considered were $T_{A}$ of (28) with $\hat{r}_{t}=\frac{1}{\hat{h}_{t}} \sum_{i=0}^{t-1} \hat{\beta}_{1}^{i} \hat{I}_{t-1-i} \hat{\varepsilon}_{t-1-i} ; T_{N}$ of (26) with $\hat{r}_{t}=\frac{1}{\hat{h}_{t}} \sum_{i=0}^{t-1} \hat{\beta}_{1}^{i} \hat{\varepsilon}_{t-1-i}^{3}$; the Engle and Ng statistic, $T_{E N}$, of (18); and the Lundbergh and Teräsvirta statistic, $T_{L T}$, of (17) with $\hat{v}_{t-1}=\hat{\varepsilon}_{t-1}^{3}$.

Table 1 reports the actual rejection frequencies, for the tests described previously, when the null is true. The results are reported for a nominal size of 5\% and where the correct model for the mean is estimated. When $\xi_{t} \sim N(0,1)$ and there are no estimation effects (i.e., $y_{t}=\varepsilon_{t}$ ), the empirical sizes for $T_{A}$ and $T_{E N}$ are close to the nominal size of $5 \%$, with the exception of low persistence volatility when the size of $T_{A}$ is $6 \%$. When there are estimation effects from the conditional mean generated as an autoregressive (AR) process, $T_{E N}$ tends to be slightly undersized for the medium and low persistence volatility models, whereas $T_{A}$ is slightly oversized for the low volatility models.

The empirical size of the nonlinearity test, $T_{N}$, is close to the nominal size, except for the low volatility persistence, whereas $T_{L T}$ is undersized in all the experiments, especially for a high persistence volatility model and Student- $t$ errors. When the conditional mean is generated as an AR process, the empirical size of $T_{N}$ is close to the nominal size, whereas that of $T_{L T}$ is lower than the nominal size of $5 \%$ for all volatility models examined and significantly so under Student- $t$ errors. By ignoring asymptotically nonnegligible estimation effects, the theoretical arguments of Section 3.1 imply that the procedures based on $T_{E N}$ or $T_{L T}$ will be asymptotically undersized, and increasingly so under excess-kurtosis; the 
Table 1. Empirical size

\begin{tabular}{|c|c|c|c|c|c|c|c|c|c|c|c|c|c|c|c|c|}
\hline & \multicolumn{4}{|c|}{$N(0,1)$} & \multicolumn{4}{|c|}{$t(7)$} & \multicolumn{4}{|c|}{$t(5)$} & \multicolumn{4}{|c|}{$t(3)$} \\
\hline & $T_{A}$ & $T_{E N}$ & $T_{N}$ & $T_{L T}$ & $T_{A}$ & $T_{E N}$ & $T_{N}$ & $T_{L T}$ & $T_{A}$ & $T_{E N}$ & $T_{N}$ & $T_{L T}$ & $T_{A}$ & $T_{E N}$ & $T_{N}$ & $T_{L T}$ \\
\hline M1-H & 4.4 & 5.2 & 5.1 & 2.6 & 4.2 & 4.1 & 4.0 & 1.7 & 5.8 & 4.4 & 2.2 & 1.0 & 5.9 & 2.7 & 2.4 & 0.5 \\
\hline M2-H & 4.5 & 4.9 & 5.6 & 2.1 & 4.4 & 3.6 & 3.8 & 1.5 & 6.2 & 4.1 & 2.5 & 0.9 & 7.2 & 2.7 & 2.8 & 0.5 \\
\hline M3-H & 4.6 & 5.0 & 5.4 & 2.5 & 4.4 & 3.7 & 3.7 & 1.6 & 6.7 & 4.0 & 2.5 & 1.0 & 7.2 & 2.6 & 2.7 & 0.5 \\
\hline M4-H & 4.9 & 5.0 & 5.4 & 2.6 & 4.0 & 3.7 & 3.7 & 1.4 & 6.1 & 4.0 & 2.4 & 1.0 & 8.0 & 2.5 & 2.6 & 0.5 \\
\hline M1-M & 4.8 & 4.7 & 4.8 & 4.0 & 4.9 & 4.0 & 4.2 & 3.1 & 5.6 & 4.1 & 2.9 & 2.0 & 5.5 & 2.9 & 2.8 & 1.1 \\
\hline M2-M & 5.0 & 4.4 & 5.2 & 4.3 & 4.4 & 4.1 & 3.8 & 3.4 & 5.1 & 3.7 & 2.8 & 2.0 & 5.9 & 2.9 & 2.7 & 1.1 \\
\hline M3-M & 5.0 & 4.6 & 5.4 & 4.3 & 4.5 & 3.9 & 4.0 & 3.4 & 5.0 & 4.0 & 2.8 & 2.0 & 6.0 & 2.9 & 2.7 & 1.1 \\
\hline M4-M & 5.1 & 4.6 & 5.4 & 4.0 & 4.4 & 3.8 & 4.2 & 3.3 & 5.3 & 4.0 & 3.1 & 2.1 & 6.7 & 3.2 & 2.6 & 1.1 \\
\hline M1-L & 6.0 & 4.7 & 4.2 & 4.1 & 4.6 & 3.8 & 3.8 & 2.6 & 4.8 & 4.1 & 2.5 & 1.4 & 6.5 & 2.0 & 1.9 & 0.5 \\
\hline M2-L & 5.7 & 4.7 & 5.3 & 3.9 & 4.7 & 3.8 & 4.0 & 2.8 & 4.0 & 4.0 & 2.6 & 1.5 & 6.4 & 1.5 & 2.3 & 0.6 \\
\hline M3-L & 5.7 & 4.4 & 5.2 & 4.0 & 4.8 & 4.0 & 4.3 & 2.5 & 4.0 & 3.8 & 2.6 & 1.6 & 6.1 & 1.8 & 2.3 & 0.7 \\
\hline M4-L & 5.7 & 4.6 & 5.2 & 3.9 & 4.6 & 4.0 & 3.7 & 2.7 & 4.6 & 4.0 & 2.5 & 1.5 & 5.8 & 1.9 & 2.1 & 0.6 \\
\hline
\end{tabular}


TABLE 2. Empirical size-adjusted power

\begin{tabular}{|c|c|c|c|c|c|c|c|c|}
\hline \multicolumn{9}{|c|}{ GJR(1,1) model } \\
\hline & \multicolumn{4}{|c|}{$N(0,1)$} & \multicolumn{4}{|c|}{$t(7)$} \\
\hline & $T_{A}$ & $T_{E N}$ & $T_{N}$ & $T_{L T}$ & $T_{A}$ & $T_{E N}$ & $T_{N}$ & $T_{L T}$ \\
\hline \multicolumn{9}{|c|}{$h_{t}=0.005+0.23\left[\left|\varepsilon_{t-1}\right|-0.23 \varepsilon_{t-1}\right]^{2}+0.7 h_{t-1}$} \\
\hline M1 & 88.7 & 47.1 & 89.2 & 16.5 & 62.2 & 32.7 & 64.9 & 14.4 \\
\hline M2 & 84.6 & 38.3 & 87.4 & 14.5 & 59.0 & 27.1 & 59.0 & 12.2 \\
\hline M3 & 84.1 & 38.5 & 87.0 & 14.2 & 59.9 & 27.2 & 58.8 & 12.6 \\
\hline M4 & 84.6 & 37.9 & 87.8 & 16.6 & 58.7 & 28.0 & 59.7 & 12.8 \\
\hline \multicolumn{9}{|c|}{$h_{t}=0.005+0.23\left[\left|\varepsilon_{t-1}\right|-0.17 \varepsilon_{t-1}\right]^{2}+0.7 h_{t-1}$} \\
\hline M1 & 67.5 & 30.0 & 67.0 & 12.4 & 40.4 & 22.5 & 42.8 & 11.6 \\
\hline M2 & 62.1 & 26.1 & 65.3 & 10.5 & 39.7 & 19.7 & 37.3 & 10.2 \\
\hline M3 & 61.3 & 26.6 & 64.6 & 10.6 & 40.5 & 19.3 & 37.1 & 10.6 \\
\hline M4 & 61.3 & 24.5 & 66.2 & 12.2 & 40.2 & 20.0 & 37.6 & 10.9 \\
\hline \multicolumn{9}{|c|}{ STGARCH(1,1) model } \\
\hline & \multicolumn{4}{|c|}{$N(0,1)$} & \multicolumn{3}{|c|}{$t(7)$} & \\
\hline & $T_{A}$ & $T_{E N}$ & $T_{N}$ & $T_{L T}$ & $T_{A}$ & $T_{E N}$ & $T_{N}$ & $T_{L T}$ \\
\hline \multicolumn{9}{|c|}{$\begin{aligned} h_{t}= & 0.005+0.136 \varepsilon_{t-1}^{2}-0.212 F\left(\varepsilon_{t-1}\right) \varepsilon_{t-1}^{2}+0.7 h_{t-1} \\
& F\left(\varepsilon_{t-1}\right)=1 /\left(1+\exp \left(-100 \varepsilon_{t-1}\right)\right)-\frac{1}{2}\end{aligned}$} \\
\hline M1 & 95.7 & 69.0 & 96.7 & 49.3 & 78.0 & 46.4 & 81.5 & 29.6 \\
\hline M2 & 95.8 & 64.7 & 97.0 & 45.9 & 75.0 & 43.4 & 80.6 & 27.1 \\
\hline M3 & 95.5 & 64.5 & 96.7 & 45.7 & 74.8 & 43.9 & 80.3 & 26.7 \\
\hline M4 & 95.2 & 63.9 & 96.5 & 47.3 & 75.9 & 45.5 & 81.3 & 26.9 \\
\hline \multicolumn{9}{|c|}{$\begin{array}{c}h_{t}=0.005+0.136 \varepsilon_{t-1}^{2}-0.17 F\left(\varepsilon_{t-1}\right) \varepsilon_{t-1}^{2}+0.7 h_{t-1} \\
F\left(\varepsilon_{t-1}\right)=1 /\left(1+\exp \left(-100 \varepsilon_{t-1}\right)\right)-\frac{1}{2}\end{array}$} \\
\hline M1 & 86.1 & 51.5 & 88.7 & 36.6 & 60.7 & 34.5 & 62.3 & 20.6 \\
\hline M2 & 84.7 & 46.9 & 88.2 & 41.8 & 59.0 & 34.0 & 62.0 & 18.9 \\
\hline M3 & 83.8 & 48.0 & 87.4 & 34.3 & 57.2 & 33.6 & 60.8 & 19.2 \\
\hline M4 & 84.1 & 46.9 & 87.2 & 35.2 & 59.1 & 34.2 & 62.3 & 19.3 \\
\hline
\end{tabular}

Continued

Monte Carlo evidence supports this, although $T_{E N}$ is "relatively" more robust than $T_{L T}$.

The results of the Monte Carlo study for assessing the size-adjusted power of the tests are reported in Table 2, where the nominal size is again 5\%. The alternative models used are the GJR $(1,1)$ model, with the parameter values considered by Lundbergh and Teräsvirta (2002) in their simulations; the logistic smooth transition, STGARCH $(1,1)$, model; the EGARCH $(1,1)$ model with parameter values 
TABLE 2. Continued

\begin{tabular}{|c|c|c|c|c|c|c|c|c|}
\hline \multicolumn{9}{|c|}{$\operatorname{EGARCH}(1,1)$ model } \\
\hline & \multicolumn{4}{|c|}{$N(0,1)$} & \multicolumn{4}{|c|}{$t(7)$} \\
\hline & $T_{A}$ & $T_{E N}$ & $T_{N}$ & $T_{L T}$ & $T_{A}$ & $T_{E N}$ & $T_{N}$ & $T_{L T}$ \\
\hline \multicolumn{9}{|c|}{$\log \left(h_{t}\right)=-0.23+0.9 \log \left(h_{t-1}\right)+0.25\left[\left|\xi_{t-1}\right|-0.3 \xi_{t-1}\right]$} \\
\hline M1 & 83.9 & 29.7 & 76.5 & 35.9 & 64.9 & 23.3 & 52.8 & 23.8 \\
\hline M2 & 82.8 & 27.2 & 75.7 & 33.7 & 64.4 & 20.1 & 49.4 & 20.3 \\
\hline M3 & 82.1 & 27.0 & 75.5 & 34.2 & 63.1 & 19.9 & 49.3 & 20.9 \\
\hline M4 & 83.2 & 27.0 & 75.1 & 32.4 & 63.0 & 19.7 & 49.3 & 21.7 \\
\hline \multicolumn{9}{|c|}{ TGARCH(1,1) model } \\
\hline & \multicolumn{4}{|c|}{$N(0,1)$} & \multicolumn{4}{|c|}{$t(7)$} \\
\hline & $T_{A}$ & $T_{E N}$ & $T_{N}$ & $T_{L T}$ & $T_{A}$ & $T_{E N}$ & $T_{N}$ & $T_{L T}$ \\
\hline \multicolumn{9}{|c|}{$\sqrt{h_{t}}=0.07+0.081\left(1-I_{t-1}\right)\left|\varepsilon_{t-1}\right|+0.193 I_{t-1}\left|\varepsilon_{t-1}\right|+0.831 \sqrt{h_{t-1}}$} \\
\hline M1 & 98.3 & 46.3 & 97.4 & 54.5 & 91.1 & 31.2 & 81.9 & 33.0 \\
\hline M2 & 98.8 & 40.4 & 96.7 & 48.5 & 90.1 & 26.6 & 80.6 & 29.3 \\
\hline M3 & 98.3 & 41.5 & 96.8 & 49.5 & 89.4 & 26.6 & 80.5 & 29.2 \\
\hline M4 & 98.9 & 40.3 & 96.9 & 49.9 & 89.6 & 27.1 & 81.0 & 29.7 \\
\hline
\end{tabular}

considered by Engle and Ng (1993); and, the TGARCH(1,1) model. In the last case, the parameter values used are estimates obtained by Zakoïan (1994) for the CAC 40 daily stock index. Note that in these experiments, for the nonlinearity tests, the "omitted" (scalar) variable is $v_{t-1}=\varepsilon_{t-1}^{3}$ when the data are generated from the GJR and STGARCH models but $v_{t-1}=\varepsilon_{t-1}$ for the EGARCH and TGARCH models. The models for the conditional mean equation are M1-M4, and we consider $\xi_{t} \sim N(0,1)$ and $\xi_{t} \sim t(7)$.

When the true data generating process is a $\operatorname{GJR}(1,1)$ model, the asymmetry test, $T_{A}$, performs remarkably well compared with the test proposed by Engle and $\mathrm{Ng}$ (1993), $T_{E N}$. This is true also when the distribution of $\xi_{t}$ is nonnormal. Similarly, for the model with larger asymmetry, and under normality, the simulated power for the nonlinearity test $T_{N}$ is $89.2 \%$, whereas that of the test proposed by Lundbergh and Teräsvirta (2002), $T_{L T}$ is $16.5 \%$, when there are no estimation effects from the conditional mean. This implies that $T_{L T}$ is relatively insensitive to this alternative model. ${ }^{13}$ Similar conclusions can be drawn for the model with smaller asymmetry.

For smooth transitions between negative to positive shocks (i.e., the true data process is generated by the $\operatorname{STGARCH}(1,1)$ model), the differences between the powers of $T_{A}$ and $T_{E N}$, and $T_{N}$ and $T_{L T}$, respectively, are quite large. When estimation effects from the conditional mean are present, say, M2, and the model 
with larger asymmetry is examined, the power of $T_{N}$ is $97 \%$, whereas that of $T_{L T}$ is $45.9 \%$. Similarly, the asymmetry test $T_{A}$ attains a simulated power of $95.8 \%$, whereas the actual rejection frequency of $T_{E N}$ is $64.7 \%$. For the nonnormal distribution, the differences are also significant.

For the other data generating processes, i.e., the $\operatorname{EGARCH}(1,1)$ and TGARCH $(1,1)$ models, the results are similar. The simulated power of the tests $T_{A}$ and $T_{N}$ is much higher than the power of the tests proposed by Engle and $\mathrm{Ng}$ (1993) and Lundbergh and Teräsvirta (2002).

Overall, the Monte Carlo simulations confirm the theoretical derivations undertaken in the previous sections. The "new" tests, namely, $T_{A}$ and $T_{N}$, have fairly good size properties and very good power when compared with $T_{E N}$ and $T_{L T}$. Moreover, the simulations reveal that these tests can be employed as general misspecification tests of asymmetry and nonlinearity because they have power against the asymmetry and/or nonlinear models proposed in the literature.

The theoretical results and Monte Carlo experiments are predicated on the correct specification for the conditional mean (regression) function. However, intuitively, because the test indicators are asymptotically sensitive to the conditional mean estimation, one might expect the corresponding procedures to be sensitive to local misspecification of $m\left(\mathbf{w}_{t} ; \boldsymbol{\varphi}\right)$. This can be investigated using the tools of Godfrey and Orme (1996) and reveals that all the tests considered here, $T_{L T}, T_{N}, T_{E N}$, and $T_{A}$, will indeed be sensitive to such misspecification (even when conditional variance is correctly specified as GARCH). Some Monte Carlo simulations ${ }^{14}$ support this finding but also suggest that $T_{N}$ and $T_{A}$ will be more robust than $T_{L T}$ and $T_{E N}$, respectively, to this form of (unconsidered) misspecification.

\section{CONCLUSION}

This paper has provided some unifying results for parametric misspecification testing in regression models with GARCH errors that have practical implications for empirical research. First, a general analytical approach has been provided for the construction of asymptotically valid test statistics that can accommodate, e.g. misspecification tests for the STAR-GARCH model, something that has not been considered in the literature to date. The principal theoretical finding from this analysis is that even under conditional symmetry, implying that the estimated conditional mean (regression) and variance parameters are asymptotically orthogonal, estimation effects from the conditional mean (regression) parameters cannot be treated as asymptotically negligible. Exploiting this, it is established that the nonlinearity and asymmetry tests proposed by Lundbergh and Teräsvirta (2002) and Engle and $\mathrm{Ng}$ (1993), respectively, are not asymptotically valid (because they ignore asymptotically nonnegligible estimation effects) and, more generally, all test procedures that erroneously neglect such estimation effects will be asymptotically undersized when the error distribution is fat-tailed. Second, new tests have been introduced for nonlinearity and asymmetry that, it is conjectured, should 
have better power properties than some existing tests against many popular alternatives to the $\operatorname{GARCH}(p, q)$ model.

The principal theoretical findings are supported by Monte Carlo results that also suggest that the new tests are quite powerful against various nonlinear models proposed in the literature, suggesting that they can be useful as general misspecification tests against nonlinearity and/or asymmetry in GARCH models. However, all procedures discussed here will (in general) be sensitive to (unconsidered) misspecification of the conditional mean (regression) function, even when the conditional variance is correctly specified, although limited Monte Carlo simulations indicate that $T_{N}$ and $T_{A}$ are less sensitive in this respect. This suggests a strategy of first testing for misspecification of the conditional mean (employing conditional-hetereroskedasticity-robust procedures). Then, after estimating the GARCH process, misspecification tests of the conditional variance can be performed using the asymptotically valid procedures proposed in this paper.

\section{NOTES}

1. Although the conditional mean estimation effects appear to have been (erroneously) assumed away the issue of estimation effects from the conditional heteroskedasticity parameters has been acknowledged; see, e.g., Li and Mak (1994) and Lundbergh and Teräsvirta (2002).

2. For example, Lundbergh and Teräsvirta (1999) proposed the STAR-GARCH model, and the statistical properties of this model were investigated by Chan and McAleer (2002).

3. As discussed by Nelson and Cao (1992), although sufficient, Assumption A3(b) is not necessary to ensure nonnegative conditional variances.

4. Weiss (1986) established the asymptotic theory for the ARCH model allowing for exogenous variables in the conditional mean.

5. Note that this is not the same start-up scheme employed by Ling and McAleer (2003), who choose $\tilde{\boldsymbol{\varepsilon}}_{0}=\mathbf{0}$, Berkes et al. (2003), or Francq and Zakoïan (2004).

6. Lundbergh and Teräsvirta (2002) employed a similar approach to test for no remaining ARCH effects, in a GARCH model, but with an implicit null of $\mathrm{E}\left[\left(\xi_{t}^{2}-1\right) \mathbf{r}_{t}^{\mathcal{G}}\right]=\mathbf{0}$, where $\mathbf{r}_{t}^{\mathcal{G}}=$ $\left(\xi_{t-1}^{2}, \ldots, \xi_{t-m}^{2}\right)^{\prime}$ is $\mathcal{G}_{t-1}^{t-m}=\sigma\left(\xi_{t-1}, \ldots, \xi_{t-m}\right)$ measurable; see Section 3.1 of Lundbergh and Teräsvirta (2002). However, this could yield tests with lower power than those based on (8), because test variables of the form $\mathbf{r}_{t}^{\mathcal{G}}$ contain less information about $\mathcal{F}_{t-1}$ than the test variables $\mathbf{r}_{t}$.

7. Assumption (ii) in Theorem 2 might require stronger moment conditions on $\varepsilon_{0 t}$ than is demanded by Assumption B1.

8. Under normality, $\boldsymbol{\Sigma}$ is the conditional variance of $\mathbf{d}_{\boldsymbol{\pi} t}^{\infty}\left(\boldsymbol{\theta}_{0}\right)$ given $\mathbf{d}_{\eta t}^{\infty}\left(\boldsymbol{\theta}_{0}\right)$ and $\mathbf{d}_{\boldsymbol{\varphi} t}^{\infty}\left(\boldsymbol{\theta}_{0}\right)$.

9. Although Bollerslev (1986, p. 318) asserts that $\hat{\boldsymbol{\varphi}}$ and $\hat{\boldsymbol{\eta}}$ are asymptotically orthogonal within a QML framework, under conditional symmetry, he does not show it. Engle (1982) provides a useful theorem, exploiting symmetry, that enables this result to be established for the ARCH model. However, because he assumes normality, the importance of the conditional symmetry assumption of $\xi_{t}$ is not stated explicitly.

10. In the proof of Theorem 2 it is established that all the test indicators considered in this section satisfy the assumptions of the paper.

11. Lundbergh and Teräsvirta (2002) obtain this statistic from a quasi-score principle, but, given the alternative entertained, the test variables should have been $\hat{\mathbf{r}}_{t}=\hat{h}_{t}^{-1} \hat{\mathbf{v}}_{t-1}$.

12. However, it can be shown that the test for remaining ARCH effects, also proposed by Lundbergh and Teräsvirta (2002), is asymptotically valid. The intuition for this is that because the alternative, being $\operatorname{GARCH}(p, q+m)$, is of the same form as the null specification, asymptotic orthogonality 
between the mean regression parameter estimators and the estimated parameters of the GARCH process ensures that inferences concerning the latter are unaffected (asymptotically) by the former. The same intuition also applies to the parameter constancy test of Lundbergh and Teräsvirta (2002), in which the alternative can be written as $h_{t}^{a}=\boldsymbol{\gamma}_{t}^{\prime} \mathbf{s}_{t-1}, \boldsymbol{\gamma}_{t}=\boldsymbol{\eta}+\sum_{i=1}^{n} t^{i} \boldsymbol{\pi}_{i}$, which is still linear in the variables of $\mathbf{s}_{t-1}$.

13. If the omitted variable $\mathbf{v}_{t-1}=\left(\varepsilon_{t-1}, \varepsilon_{t-1}^{3}\right)^{\prime}$ is considered as in the Monte Carlo study of Lundbergh and Teräsvirta (2002) and for a nominal size of $10 \%$, then the size-adjusted powers are $95.3 \%$ for $T_{N}$ and $65.1 \%$ for $T_{L T}$.

14. These Monte Carlo simulations are not reported here but are available from the authors upon request.

\section{REFERENCES}

Bai, J. \& S. Ng (2001) A consistent test for conditional symmetry in time series models. Journal of Econometrics 103, 225-258.

Berkes, I., L. Horváth, \& P. Kokoszka (2003) GARCH processes: Structure and estimation. Bernoulli 9, 201-227.

Bollerslev, T. (1986) Generalized autoregressive conditional heteroskedasticity. Journal of Econometrics 31, 307-327.

Chan, F. \& M. McAleer (2002) Maximum likelihood estimation of STAR and STAR-GARCH models: Theory and Monte Carlo evidence. Journal of Applied Econometrics 17, 509-534.

Chan, F. \& M. McAleer (2003) On the Structure, Asymptotic Theory and Applications of STARGARCH Models. CIRJE-F-216 Discussion paper, University of Western Australia.

Cox, D.R. \& N. Reid (1987) Parameter orthogonality and approximate conditional inference. Journal of the Royal Statistical Society, Series B 49, 1-39 (with discussion).

Durbin, J. (1970) Testing for serial correlation in least squares regression when some of the regressors are lagged dependent variables. Econometrica 38, 410-421.

Engle, R.F. (1982) Autoregressive conditional heteroskedasticity, with estimates of the variance of United Kingdom inflation. Econometrica 50, 987-1008.

Engle, R.F. \& V.K. Ng (1993) Measuring and testing the impact of news on volatility. Journal of Finance 48, 1749-1777.

Francq, C. \& J.-M. Zakoïan (2004) Maximum likelihood estimation of pure GARCH and ARMAGARCH processes. Bernoulli 10, 605-637.

Francq, C. \& J.-M. Zakoïan (2007) Quasi-maximum likelihood estimation in GARCH processes when some coefficients are equal to zero. Stochastic Processes and Their Applications 117, 1265-1284.

Glosten, L., R. Jagannathan, \& D. Runkle (1993) On the relation between expected value and the volatility of nominal excess returns on stocks. Journal of Finance 48, 1779-1801.

Godfrey, L.G. (1996) Some results on the Glejser and Koenker tests for heteroskedasticity. Journal of Econometrics 72, 275-299.

Godfrey, L.G. \& C.D. Orme (1996) On the behavior of conditional moment tests in the presence of unconsidered local alternatives. International Economic Review 37, 263-281.

Gonzalez-Rivera, G. (1998) Smooth transition GARCH models. Studies in Nonlinear Dynamics and Econometrics 3, 61-78.

Hagerud, G.E. (1997) A Smooth Transition ARCH Model for Asset Returns. SSE/EFI Working Paper Series in Economics and Finance, 162, Stockholm School of Economics.

Li, W.K. \& T.K. Mak (1994) On the squared residual autocorrelations in non-linear time series with conditional heteroskedasticity. Journal of Time Series Analysis 15, 627-636.

Ling, S. \& M. McAleer (2003) Asymptotic theory for a vector ARMA-GARCH model. Econometric Theory 19, 280-310.

Lundbergh, S. \& T. Teräsvirta (1999) Modelling Economic High Frequency Time Series with STARSTGARCH models. SSE/EFI Working Paper Series in Economics and Finance, 390, Stockholm School of Economics. 
Lundbergh, S. \& T. Teräsvirta (2002) Evaluating GARCH models. Journal of Econometrics 110, 417-435.

Nelson, D.B. (1991) Conditional heteroskedasticity in asset returns: A new approach. Econometrica $59,347-370$.

Nelson, D.B. \& C.Q. Cao (1992) Inequality constraints in the univariate GARCH model. Journal of Business \& Economic Statistics 10, 229-235.

Newey, W.K. (1985) Maximum likelihood specification testing and conditional moment tests. Econometrica 53, 1047-1070.

Weiss, A.A. (1986) Asymptotic theory for ARCH models: Estimation and testing. Econometric Theory $2,107-131$.

Wooldridge, J. (1991) On the application of robust, regression-based diagnostics to models of conditional means and conditional variances. Journal of Econometrics 47, 5-46.

Zakoïan, J.-M. (1994) Threshold heteroskedastic models. Journal of Economic Dynamics and Control $18,931-955$.

\section{APPENDIX A}

We shall exploit the results contained in the following three preliminary propositions.

\section{PROPOSITION 2.}

(i) For any vector $\mathbf{c} \in \Re^{r},\left(\partial m\left(\mathbf{w}_{t} ; \boldsymbol{\varphi}\right) / \partial \boldsymbol{\varphi}^{\prime}\right) \mathbf{c}=0$, almost surely (a.s.), only if $\mathbf{c}=\mathbf{0}$.

(ii) For any vector $\mathbf{b} \in \Re^{p+q+1},\left(\partial h_{t}^{\infty} / \partial \boldsymbol{\eta}^{\prime}\right) \mathbf{b}=0$, a.s., only if $\mathbf{b}=\mathbf{0}$.

Proof. The proof of (i) follows immediately from Assumption B2, which implies that $\left|m\left(\mathbf{w}_{t} ; \boldsymbol{\varphi}\right)-m\left(\mathbf{w}_{t} ; \boldsymbol{\varphi}_{0}\right)\right|^{2}>0$, a.s., for all $\boldsymbol{\varphi} \neq \boldsymbol{\varphi}_{0}$. Then, by Assumption B3 and a mean value expansion, $\left(\boldsymbol{\varphi}-\boldsymbol{\varphi}_{0}\right)^{\prime}\left(\partial m\left(\mathbf{w}_{t} ; \overline{\boldsymbol{\varphi}}\right) / \partial \boldsymbol{\varphi}\right)\left(\partial m\left(\mathbf{w}_{t} ; \overline{\boldsymbol{\varphi}}\right) / \partial \boldsymbol{\varphi}^{\prime}\right)\left(\boldsymbol{\varphi}-\boldsymbol{\varphi}_{0}\right)>0$, a.s., for all $\boldsymbol{\varphi} \neq \boldsymbol{\varphi}_{0}$ and some mean value $\overline{\boldsymbol{\varphi}}$. Correspondingly, the identification condition, A3(c), establishes (ii); see, e.g., Ling and McAleer (2003) or Berkes et al. (2003).

PROPOSITION 3. Under Assumptions B1 and B3(a), $\operatorname{Esup}_{\boldsymbol{\theta} \in \Theta}\left|\varepsilon_{t}\right|^{4(1+s)}<\infty$, for some $s>0$, uniformly in $t$.

Proof. Let $m_{t} \equiv m\left(\mathbf{w}_{t} ; \boldsymbol{\varphi}\right)$ and $m_{0 t} \equiv m\left(\mathbf{w}_{t} ; \boldsymbol{\varphi}_{0}\right)$, so that $\varepsilon_{t} \equiv \varepsilon_{t}(\boldsymbol{\theta})=\varepsilon_{0 t}-\left(m_{t}-m_{0 t}\right)$. By Assumptions B1 and B3(a) and the $c_{r}$-inequality, for some constant $C>0$ and $0<r \leq$ $4(1+s)$

$\underset{\theta}{\mathrm{E} \sup _{\boldsymbol{\theta}}}\left|\varepsilon_{t}\right|^{r} \leq C\left(\mathrm{E}\left|\varepsilon_{0 t}\right|^{r}+2^{r} \mathrm{E}\left|B\left(\mathbf{w}_{t}\right)\right|\right)<\infty$.

DEFINITION 1. In the following exposition $C, K$, and $\rho$ denote generic constants, independent of $\boldsymbol{\theta}$, whose values might change from expression to expression but that always satisfy $C>0, K>0$, and $0<\rho<1$.

\section{Remark 1.}

(a) By Assumption A3(b), for all $r>0, \operatorname{Esup}_{\boldsymbol{\theta}}\left|a_{t}\right|^{r}<\infty$, provided $\operatorname{Esup}_{\boldsymbol{\theta}}\left|\varepsilon_{t}\right|^{2 r}<\infty$, uniformly in $t$. 
(b) The following inequalities will be useful: (1) $h_{t}^{\infty}=\sum_{i=0}^{\infty} \psi_{i} a_{t-i} \geq \lambda>0$; (2) $h_{t}^{\infty} \geq$ $\alpha_{0}+\psi_{i} a_{t-i}, i \geq 1$; or (3) $h_{t}^{\infty} \geq \alpha_{0}+\psi_{i} \alpha_{m} \varepsilon_{t-i-m}^{2}, i \geq 0, m=1, \ldots, q$. Given the construction of initial values, $\tilde{\varepsilon}_{0}$, we can also write (1) $h_{t}=\sum_{i=0}^{t-1} \psi_{i} a_{t-i} \geq \lambda>0$; (2) $h_{t} \geq$ $\alpha_{0}+\psi_{i} a_{t-i}, i=1, \ldots, t-1$; or (3) $h_{t} \geq \alpha_{0}+\psi_{i} \alpha_{m} \varepsilon_{t-i-m}^{2}, i=0, \ldots, t-1, m=1, \ldots, q$.

(c) The proofs will exploit the following results, which follow from Berkes et al. (2003) and Francq and Zakoïan (2004). A particularly useful device, in this respect, is $x /(1+x) \leq$ $x^{s}$, for all $x>0$ and any $s \in(0,1)$.

$$
\begin{aligned}
& \left|h_{t}^{\infty}-h_{t}\right|=\sum_{i=t}^{\infty} \psi_{i}\left|a_{t-i}\right| \leq K \sum_{i=t}^{\infty} \rho^{i}\left|a_{t-i}\right|, \\
& \left|\frac{h_{t}^{\infty}-h_{t}}{h_{t}^{\infty}}\right| \leq \sum_{i=t}^{\infty} \frac{\psi_{i} a_{t-i}}{\alpha_{0}+\psi_{i} a_{t-i}} \leq K \sum_{i=t}^{\infty} \rho^{i} a_{t-i}^{s} .
\end{aligned}
$$

For $r=1,2,3$,

$$
\left|\frac{1}{\left(h_{t}^{\infty}\right)^{r}}-\frac{1}{\left(h_{t}\right)^{r}}\right| \leq C\left|\frac{h_{t}^{\infty}-h_{t}}{h_{t}^{\infty}}\right| \leq K \sum_{i=t}^{\infty} \rho^{i} a_{t-i}^{s} .
$$

Let $\nabla_{\boldsymbol{\theta}}, \nabla_{\boldsymbol{\theta} \boldsymbol{\theta}}$ denote first- and second-order differentiation, respectively; e.g., $\mathbf{x}_{t}^{\infty}=$ $\left(1 / h_{t}^{\infty}\right) \nabla_{\boldsymbol{\eta}} h_{t}^{\infty}, \nabla_{\boldsymbol{\varphi} \boldsymbol{\eta}} h_{t}^{\infty}=\frac{\partial^{2} h_{t}^{\infty}}{\partial \boldsymbol{\varphi} \partial \boldsymbol{\eta}^{\prime}}$, etc. Then, by Assumption A3(b),

$\left\|\mathbf{x}_{t}^{\infty}\right\| \leq K\left\{1+\sum_{i=1}^{\infty} i \rho^{i} a_{t-i}^{s}\right\} ;\left\|\mathbf{x}_{t}\right\| \leq K\left\{1+\sum_{i=1}^{t-1} i \rho^{i} a_{t-i}^{s}\right\}$,

$\left\|\frac{1}{h_{t}^{\infty}} \nabla_{\eta} h_{t}^{\infty}\right\| \leq K\left\{1+\sum_{i=2}^{\infty} i^{2} \rho^{i} a_{t-i}^{s}\right\}$

$\left\|\frac{1}{h_{t}} \nabla_{\boldsymbol{\eta}} h_{t}\right\| \leq K\left\{1+\sum_{i=2}^{t-1} i^{2} \rho^{i} a_{t-i}^{s}\right\}$

$\frac{1}{h_{t}^{\infty}}\left\|\nabla_{\boldsymbol{\eta}} h_{t}^{\infty}-\nabla_{\boldsymbol{\eta}} h_{t}\right\| \leq K\left\{\rho^{t}+\sum_{i=t}^{\infty} i \rho^{i} a_{t-i}^{s}\right\}$,

$\frac{1}{h_{t}^{\infty}}\left\|\nabla_{\eta \eta} h_{t}^{\infty}-\nabla_{\eta \eta} h_{t}\right\| \leq K\left\{t \rho^{t}+\sum_{i=t}^{\infty} i^{2} \rho^{i} a_{t-i}^{s}\right\}$.

Define $d_{t}=\sum_{k=1}^{q}\left\|\frac{\partial m_{t-k}}{\partial \boldsymbol{\varphi}}\right\|=\sum_{k=1}^{q}\left\|\mathbf{f}_{t-k}\right\|$ and $g_{t}=\sum_{k=1}^{q}\left\{\left\|\mathbf{f}_{t-k}\right\|^{2}+\left\|\frac{\partial^{2} m_{t-k}}{\partial \boldsymbol{\varphi} \partial \boldsymbol{\varphi}^{\prime}}\right\|\right\}$. Then by similar methods, and using $0 \leq x /\left(1+x^{2}\right)<1$ for all $x \geq 0$, it can also be shown that $\left\|\mathbf{c}_{t}^{\infty}\right\| \leq K \sum_{i=0}^{\infty} \rho^{i} d_{t-i} ; \quad\left\|\mathbf{c}_{t}\right\| \leq K \sum_{i=0}^{t-1} \rho^{i} d_{t-i}$ 
$\left\|\frac{1}{h_{t}^{\infty}} \nabla_{\varphi \varphi} h_{t}^{\infty}\right\| \leq K \sum_{i=0}^{\infty} \rho^{i} g_{t-i} ; \quad\left\|\frac{1}{h_{t}} \nabla_{\varphi \varphi} h_{t}\right\| \leq K \sum_{i=0}^{t-1} \rho^{i} g_{t-i}$

$$
\frac{1}{h_{t}^{\infty}}\left\|\nabla_{\varphi} h_{t}^{\infty}-\nabla_{\varphi} h_{t}\right\| \leq K \sum_{i=t}^{\infty} \rho^{i} d_{t-i}
$$

$\frac{1}{h_{t}^{\infty}}\left\|\nabla_{\varphi \varphi} h_{t}^{\infty}-\nabla_{\varphi \varphi} h_{t}\right\| \leq K \sum_{i=t}^{\infty} \rho^{i} g_{t-i}$

$\left\|\frac{1}{h_{t}^{\infty}} \nabla_{\varphi} h_{t}^{\infty}\right\| \leq K\left\{d_{t}+\sum_{i=1}^{\infty} i \rho^{i} d_{t-i}\right\}$

$\left\|\frac{1}{h_{t}} \nabla_{\varphi \eta} h_{t}\right\| \leq K\left\{d_{t}+\sum_{i=1}^{t-1} i \rho^{i} d_{t-i}\right\}$

$\frac{1}{h_{t}^{\infty}}\left\|\nabla_{\varphi \eta} h_{t}^{\infty}-\nabla_{\varphi \eta} h_{t}\right\| \leq K \sum_{i=t}^{\infty} i \rho^{i} d_{t-i}$

$\frac{1}{h_{t}^{\infty 2}}\left\|\frac{\partial h_{t}^{\infty}}{\partial \boldsymbol{\varphi}} \frac{\partial h_{t}^{\infty}}{\partial \varphi^{\prime}}-\frac{\partial h_{t}}{\partial \boldsymbol{\varphi}} \frac{\partial h_{t}}{\partial \boldsymbol{\varphi}^{\prime}}\right\| \leq K \sum_{i=t}^{\infty} \rho^{i} d_{t-i}\left\{\sum_{i=0}^{\infty} \rho^{i} d_{t-i}\right\}$,

$\frac{1}{h_{t}^{\infty 2}}\left\|\frac{\partial h_{t}^{\infty}}{\partial \boldsymbol{\eta}} \frac{\partial h_{t}^{\infty}}{\partial \boldsymbol{\eta}^{\prime}}-\frac{\partial h_{t}}{\partial \boldsymbol{\eta}} \frac{\partial h_{t}}{\partial \boldsymbol{\eta}^{\prime}}\right\| \leq K\left\{\rho^{t}+\sum_{i=t}^{\infty} i \rho^{i} a_{t-i}^{s}\right\}\left\{1+\sum_{i=1}^{\infty} i \rho^{i} a_{t-i}^{s}\right\}$,

$\frac{1}{h_{t}^{\infty 2}}\left\|\frac{\partial h_{t}^{\infty}}{\partial \boldsymbol{\varphi}} \frac{\partial h_{t}^{\infty}}{\partial \boldsymbol{\eta}^{\prime}}-\frac{\partial h_{t}}{\partial \boldsymbol{\varphi}} \frac{\partial h_{t}}{\partial \boldsymbol{\eta}^{\prime}}\right\| \leq K \sum_{i=t}^{\infty} \rho^{i} d_{t-i}\left\{1+\sum_{i=1}^{\infty} i \rho^{i} a_{t-i}^{s}\right\}$

$$
+\left\{\rho^{t}+\sum_{i=t}^{\infty} i \rho^{i} a_{t-i}^{s}\right\} \sum_{i=0}^{\infty} \rho^{i} d_{t-i}
$$

where in (A.13) we have used, for conformable $\mathbf{x}, \mathbf{y}, \mathbf{a}$, and $\mathbf{b}$,

$$
\begin{aligned}
\left\|\mathbf{x} \mathbf{a}^{\prime}-\mathbf{y} \mathbf{b}^{\prime}\right\| & \leq\|\mathbf{x}-\mathbf{y}\|\|\mathbf{a}\|+\|\mathbf{a}-\mathbf{b}\|\|\mathbf{y}\|, \\
\text { or } \quad\left\|\mathbf{x} \mathbf{a}^{\prime}-\mathbf{y} \mathbf{b}^{\prime}\right\| & \leq\|\mathbf{a}-\mathbf{b}\|\|\mathbf{x}\|+\|\mathbf{x}-\mathbf{y}\|\|\mathbf{b}\| .
\end{aligned}
$$

Because $h_{t} / h_{t}^{\infty} \leq 1$, for conformable matrices $\mathbf{A}$ and $\mathbf{B}$, and $p=1,3 / 2,2,3$,

$$
\left\|\frac{1}{\left(h_{t}^{\infty}\right)^{p}} \mathbf{A}-\frac{1}{\left(h_{t}\right)^{p}} \mathbf{B}\right\| \leq K\left\{\frac{1}{\left(h_{t}^{\infty}\right)^{p}}\|\mathbf{A}-\mathbf{B}\|+\left\|\frac{1}{h_{t}^{p}} \mathbf{B}\right\|\left|\frac{h_{t}^{\infty}-h_{t}}{h_{t}^{\infty}}\right|\right\} .
$$

PROPOSITION 4. Under Assumptions A and B, and exploiting (A.2)-(A.14), the following moments are bounded uniformly in $t$ : 
(i) $\operatorname{Esup}_{\boldsymbol{\theta}}\left|\varepsilon_{t}\right|^{r}\left\|\frac{1}{h_{t}^{\infty}} \nabla_{\boldsymbol{\theta}} h_{t}^{\infty}\right\|^{2}, 0 \leq r \leq 4$,

(ii) $\operatorname{Esup}_{\boldsymbol{\theta}}\left|\varepsilon_{t}\right|^{r}\left\|\frac{1}{h_{t}^{\infty}} \nabla_{\boldsymbol{\theta} \boldsymbol{\theta}} h_{t}^{\infty}\right\|, r=0,2$, whereas the following moments are $O\left(\rho^{t}\right)$, at most:

(iii) $\operatorname{Esup}_{\boldsymbol{\theta}}\left\|\varepsilon_{t}^{r} \mathbf{f}_{t}\right\|\left\|\frac{1}{\left(h_{t}^{\infty}\right)^{p}} \nabla_{\boldsymbol{\theta}} h_{t}^{\infty}-\frac{1}{h_{t}^{p}} \nabla_{\boldsymbol{\theta}} h_{t}\right\|, r=0,1, p=1,3 / 2,2$,

(iv) $\operatorname{Esup}_{\boldsymbol{\theta}}\left|\varepsilon_{t}\right|^{r}\left\|\frac{1}{\left(h_{t}^{\infty}\right)^{p}} \nabla_{\boldsymbol{\theta} \boldsymbol{\theta}} h_{t}^{\infty}-\frac{1}{h_{t}^{p}} \nabla_{\boldsymbol{\theta} \boldsymbol{\theta}} h_{t}\right\|, r=0,2, p=1,2$,

(v) $\operatorname{Esup}_{\boldsymbol{\theta}}\left|\varepsilon_{t}\right|^{r}\left\|\frac{1}{\left(h_{t}^{\infty}\right)^{p}}\left(\nabla_{\boldsymbol{\theta}} h_{t}^{\infty}\right)\left(\nabla_{\boldsymbol{\theta}} h_{t}^{\infty}\right)^{\prime}-\frac{1}{h_{t}^{p}}\left(\nabla_{\boldsymbol{\theta}} h_{t}\right)\left(\nabla_{\boldsymbol{\theta}} h_{t}\right)^{\prime}\right\|, r=0,2, p=2,3$.

Proof. (i) and (ii) follow in a straightforward manner from (A.5), (A.6), (A.8), (A.9), and (A.11) and the fact that the following moments are bounded: $\operatorname{Esup}_{\boldsymbol{\theta}}\left\|\varepsilon_{t}^{4} \mathbf{f}_{t-k} \mathbf{f}_{t-j}^{\prime}\right\|$, $\operatorname{Esup}_{\boldsymbol{\theta}}\left\|\varepsilon_{t}^{2} \nabla_{\boldsymbol{\theta}} \mathbf{f}_{t-k}\right\|, \operatorname{Esup}_{\boldsymbol{\theta}}\left|\varepsilon_{t}^{4} \varepsilon_{t-j}^{2 s} \varepsilon_{t-k}^{2 s}\right|, s \in(0,1)$. For example, by an application of Hölder's inequality and then Cauchy-Schwarz inequality,

$\operatorname{Esup}_{\boldsymbol{\theta}}\left|\varepsilon_{t}^{4} \varepsilon_{t-j}^{2 s} \varepsilon_{t-k}^{2 s}\right| \leq\left(\operatorname{Esup}_{\boldsymbol{\theta}}\left|\varepsilon_{t}\right|^{4(1+s)}\right)^{1 /(1+s)}\left(\underset{\boldsymbol{\theta}}{\operatorname{Esup}}\left|\varepsilon_{t-j} \varepsilon_{t-k}\right|^{2(1+s)}\right)^{s /(1+s)}<\infty$

because, for some $s \in(0,1), \operatorname{Esup}_{\theta}\left|\varepsilon_{t}\right|^{4(1+s)}<\infty$. For (iii) use (A.14), (A.10), (A.7), (A.8), (A.5), (A.3), and the fact that $\operatorname{Esup}_{\boldsymbol{\theta}}\left\|\varepsilon_{t} \mathbf{f}_{t} \mathbf{f}_{t-k}^{\prime}\right\|, \quad \sup _{\boldsymbol{\theta}}\left\|\varepsilon_{t} \mathbf{f}_{t} \mathbf{f}_{t-k}^{\prime} \varepsilon_{t-j}^{2 s}\right\|$, $\operatorname{Esup}_{\boldsymbol{\theta}}\left\|\varepsilon_{t} \mathbf{f}_{t} \varepsilon_{t-k}^{2 s}\right\|, \operatorname{Esup}_{\boldsymbol{\theta}}\left\|\varepsilon_{t} \mathbf{f}_{t} \varepsilon_{t-k}^{2 s} \varepsilon_{t-j}^{2 s}\right\|$ are all bounded, by Cauchy-Schwarz and/or Hölder's inequality, for $s \in(0,1)$. In particular,

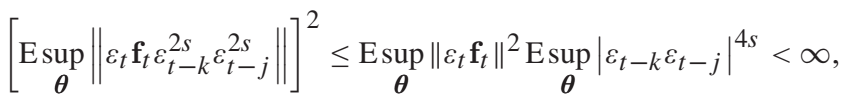

because, by Hölder's inequality and $4 s(1+s) \leq 4(1+s), s \in(0,1)$,

$\operatorname{Esup}_{\boldsymbol{\theta}}\left|\varepsilon_{t-k} \varepsilon_{t-j}\right|^{4 s} \leq\left(\operatorname{Esup}_{\boldsymbol{\theta}}\left|\varepsilon_{t-k}\right|^{4 s(1+s)}\right)^{1 /(1+s)}\left(\operatorname{Esup}_{\boldsymbol{\theta}}\left|\varepsilon_{t-j}\right|^{4(1+s)}\right)^{s /(1+s)}<\infty$.

Similarly (iv) holds because all the following moments are bounded: $\operatorname{Esup}_{\boldsymbol{\theta}}\left|\varepsilon_{t}\right|^{2}\left|\varepsilon_{t-j}\right|^{2 s}$ $\left\|\mathbf{f}_{t-k}\right\|^{2}, \operatorname{Esup}_{\boldsymbol{\theta}}\left|\varepsilon_{t}\right|^{2}\left|\varepsilon_{t-j}\right|^{2 s}\left\|\nabla_{\boldsymbol{\varphi}} \mathbf{f}_{t-k}\right\|, s \in(0,1)$. In particular,

$\left[\operatorname{Esup}_{\boldsymbol{\theta}}\left|\varepsilon_{t}\right|^{2}\left|\varepsilon_{t-j}\right|^{2 s}\left\|\mathbf{f}_{t-k}\right\|^{2}\right]^{2} \leq \operatorname{Esup}_{\boldsymbol{\theta}}\left\|\varepsilon_{t}^{2} \mathbf{f}_{t-k}\right\|^{2} \underset{\boldsymbol{\theta}}{\operatorname{Esup}}\left\|\varepsilon_{t-j}^{2 s} \mathbf{f}_{t-k}\right\|^{2}<\infty$,

$\left[\operatorname{Esup}_{\boldsymbol{\theta}}\left|\varepsilon_{t}\right|^{2}\left|\varepsilon_{t-j}\right|^{2 s}\left\|\nabla_{\boldsymbol{\varphi}} \mathbf{f}_{t-k}\right\|\right]^{2} \leq \operatorname{Eup}_{\boldsymbol{\theta}}\left\|\varepsilon_{t} \nabla_{\boldsymbol{\varphi}} \mathbf{f}_{t-k}\right\|^{2} \operatorname{Esup}_{\boldsymbol{\theta}}\left|\varepsilon_{t} \varepsilon_{t-j}^{2 s}\right|^{2}<\infty$.

Finally, (v) follows in a similar manner, noting that the following moments are bounded: $\operatorname{Esup}_{\boldsymbol{\theta}}\left\|\varepsilon_{t}^{2} \mathbf{f}_{t-k} \varepsilon_{t-j}^{2 s} \varepsilon_{t-l}^{2 s}\right\|, \operatorname{Esup}_{\boldsymbol{\theta}}\left|\varepsilon_{t}^{2} \varepsilon_{t-j}^{2 s} \varepsilon_{t-k}^{2 s} \varepsilon_{t-l}^{2 s}\right|, s \in(0,1)$. In particular, 


$$
\begin{aligned}
& {\left[\underset{\boldsymbol{\theta}}{\operatorname{Esup}}\left\|\varepsilon_{t}^{2} \mathbf{f}_{t-k} \varepsilon_{t-j}^{2 s} \varepsilon_{t-l}^{2 s}\right\|\right]^{2} \leq\left(\underset{\boldsymbol{\theta}}{\operatorname{Esup}}\left\|\varepsilon_{t}^{2} \mathbf{f}_{t-k}\right\|^{2}\right)\left(\underset{\boldsymbol{\theta}}{\operatorname{Esup}}\left|\varepsilon_{t-k} \varepsilon_{t-l}\right|^{4 s}\right)<\infty} \\
& {\left[\underset{\boldsymbol{\theta}}{\operatorname{Esup}}\left|\varepsilon_{t}^{2} \varepsilon_{t-j}^{2 s} \varepsilon_{t-k}^{2 s} \varepsilon_{t-l}^{2 s}\right|\right]^{2} \leq\left(\underset{\boldsymbol{\theta}}{\operatorname{Esup}}\left|\varepsilon_{t}^{4} \varepsilon_{t-j}^{4 s}\right|\right)\left(\underset{\theta}{\operatorname{Esup}}\left|\varepsilon_{t-k} \varepsilon_{t-l}\right|{ }^{4 s}\right)<\infty}
\end{aligned}
$$

and, by Hölder's inequality,

$\operatorname{Esup}_{\boldsymbol{\theta}}\left|\varepsilon_{t}^{4} \varepsilon_{t-j}^{4 s}\right| \leq\left(\operatorname{Esup}_{\boldsymbol{\theta}}\left|\varepsilon_{t}\right|^{4(1+s)}\right)^{1 /(1+s)}\left(\operatorname{Eup}_{\boldsymbol{\theta}}\left|\varepsilon_{t-j}\right|^{4(1+s)}\right)^{s /(1+s)}<\infty$.

The following three propositions follow the approach of Ling and McAleer (2003), Berkes et al. (2003), and Francq and Zakoïan (2004) and are used to establish the consistency and asymptotic normality of the QMLE $\hat{\boldsymbol{\theta}}$.

PROPOSITION 5. Under Assumptions A, B1, B2, and B3(a):

(i) $\mathrm{E}\left[l_{t}^{\infty}(\boldsymbol{\theta})\right]$ exists for all $\boldsymbol{\theta} \in \boldsymbol{\Theta}$.

(ii) $\sup _{\boldsymbol{\theta} \in \boldsymbol{\Theta}}\left|L_{T}^{\infty}(\boldsymbol{\theta})-\mathrm{E}\left[l_{t}^{\infty}(\boldsymbol{\theta})\right]\right|=o_{p}(1)$.

(iii) $\mathrm{E}\left[l_{t}^{\infty}(\boldsymbol{\theta})\right]$ achieves a unique maximum at $\boldsymbol{\theta}_{0}$.

(iv) $\sup _{\boldsymbol{\theta} \in \Theta}\left|L_{T}^{\infty}(\boldsymbol{\theta})-L_{T}(\boldsymbol{\theta})\right|=o_{p}(1)$.

\section{Proof.}

(i) First, by Assumption A3(b), $h_{t}^{\infty} \geq \lambda>0$, uniformly in $\boldsymbol{\theta}$; therefore, $\operatorname{Esup}_{\boldsymbol{\theta} \in \Theta} \times$ $\left|\varepsilon_{t}^{2} / h_{t}^{\infty}\right| \leq \lambda^{-1} \operatorname{Esup}_{\boldsymbol{\theta}}\left|\varepsilon_{t}\right|^{2}<\infty$, by Proposition 3. Second, by Assumption A3, $\left|h_{t}^{\infty}\right| \leq$ $K \sum_{i=0}^{\infty} \rho^{i}\left|a_{t-i}\right|$. Thus, $\operatorname{Esup}_{\boldsymbol{\theta}}\left|h_{t}^{\infty}\right|<\infty$, and by Jensen's inequality $\operatorname{Esup}_{\boldsymbol{\theta}}|\ln | h_{t}^{\infty}|| \leq$ $\left|\ln \operatorname{Esup}_{\boldsymbol{\theta}}\right| h_{t}^{\infty}||<\infty$, so that $\mathrm{E}\left[l_{t}^{\infty}(\boldsymbol{\theta})\right]$ exists for all $\boldsymbol{\theta} \in \Theta$.

(ii) By a uniform law of large numbers (ULLN) (e.g., Ling and McAleer 2003, Thm. 3.1, p. 287), it follows that $\sup _{\boldsymbol{\theta} \in \Theta}\left|L_{T}^{\infty}(\boldsymbol{\theta})-\mathrm{E}\left[l_{t}^{\infty}(\boldsymbol{\theta})\right]\right|=o_{p}(1)$.

(iii) Write

$$
\begin{aligned}
2 \mathrm{E}\left[l_{t}^{\infty}(\boldsymbol{\theta})\right] & =\left\{-\mathrm{E}\left[\ln \left(h_{t}^{\infty}\right)\right]-\mathrm{E}\left[\varepsilon_{0 t}^{2} / h_{t}^{\infty}\right]\right\}-\left\{\mathrm{E}\left[\left(m_{t}-m_{0 t}\right)^{2} / h_{t}^{\infty}\right]\right\} \\
& =\left\{L_{1}(\boldsymbol{\theta})\right\}+\left\{L_{2}(\boldsymbol{\theta})\right\}
\end{aligned}
$$

because $\mathrm{E}\left[\varepsilon_{0 t} m_{t} / h_{t}^{\infty} \mid \mathcal{F}_{t-1}\right]=0$. First, $L_{2}(\boldsymbol{\theta})=-\left\{\mathrm{E}\left[\left(m_{t}-m_{0 t}\right)^{2} / h_{t}^{\infty}\right]\right\}$ achieves a maximum value of zero only when $m_{t}=m_{0 t}$, for all $t$ a.s., which, by Assumption B2, holds only if $\varphi=\varphi_{0}$. Second (and as argued by Ling and McAleer, 2003, Lem. 4.4) using Proposition 2(ii) and given $\boldsymbol{\varphi}=\boldsymbol{\varphi}_{0}, L_{1}(\boldsymbol{\theta})$ achieves a maximum only if $\boldsymbol{\eta}=\boldsymbol{\eta}_{0}$. Thus $\mathrm{E}\left[l_{t}^{\infty}(\boldsymbol{\theta})\right]$ achieves its unique maximum at $\boldsymbol{\theta}=\boldsymbol{\theta}_{0}$.

(iv) We have

$$
2\left|L_{T}^{\infty}(\boldsymbol{\theta})-L_{T}(\boldsymbol{\theta})\right| \leq T^{-1} \sum_{t=1}^{T}\left|\ln \frac{h_{t}^{\infty}}{h_{t}}\right|+T^{-1} \sum_{t=1}^{T}\left|\varepsilon_{t}^{2}\left(\frac{1}{h_{t}^{\infty}}-\frac{1}{h_{t}}\right)\right| .
$$

Equation (A.2) and $\ln (x) \leq x-1$, for all $x>0$, yield $\operatorname{Esup}_{\boldsymbol{\theta} \in \Theta}\left|\ln \left(h_{t}^{\infty} / h_{t}\right)\right| \leq \lambda^{-1}$ $\operatorname{Esup}_{\boldsymbol{\theta} \in \Theta}\left|h_{t}^{\infty}-h_{t}\right|=O\left(\rho^{t}\right)$, at most. Therefore $T^{-1} \sum_{t=1}^{T} \operatorname{Esup}_{\boldsymbol{\theta} \in \Theta}\left|\ln \left(h_{t}^{\infty} / h_{t}\right)\right|=o(1)$, 
implying (by Markov's inequality) $T^{-1} \sum_{t=1}^{T} \sup _{\boldsymbol{\theta}}\left|\ln \left(h_{t}^{\infty} / h_{t}\right)\right|=o_{p}(1)$. Next, $T^{-1} \sum_{t=1}^{T} \sup _{\boldsymbol{\theta} \in \Theta}\left|\varepsilon_{t}^{2}\left(\left(1 / h_{t}^{\infty}\right)-\left(1 / h_{t}\right)\right)\right|=T^{-1} \sum_{t=1}^{T} X_{t} u_{t}$ where $X_{t}=\sup _{\boldsymbol{\theta}} \varepsilon_{t}^{2}$ is strictly stationary and ergodic with $\mathrm{E}\left|X_{t}\right|<\infty$ and $\left|u_{t}\right| \leq 2 \lambda^{-1}$. By (A.4), $\operatorname{Esup}_{\boldsymbol{\theta} \in \Theta} \mid\left(1 / h_{t}^{\infty}\right)-$ $\left(1 / h_{t}\right) \mid=O\left(\rho^{t}\right)$, so that $T^{-1} \sum_{t=1}^{T} u_{t}=o_{p}(1)$ and $T^{-1} \sum_{t=1}^{T} \sup _{\boldsymbol{\theta} \in \Theta} \mid \varepsilon_{t}^{2}\left(\left(1 / h_{t}^{\infty}\right)-\right.$ $\left.\left(1 / h_{t}\right)\right) \mid=o_{p}(1)$, applying Lemma 4.5 of Ling and McAleer (2003, p. 288). This completes the proof.

PROPOSITION 6. Under Assumptions $A$ and B

(i) $\boldsymbol{\Omega}_{\boldsymbol{\theta} \boldsymbol{\theta}} \equiv \boldsymbol{\Omega}_{\boldsymbol{\theta} \boldsymbol{\theta}}\left(\boldsymbol{\theta}_{0}\right)$ is finite and positive definite, where $\boldsymbol{\Omega}_{\boldsymbol{\theta} \boldsymbol{\theta}}(\boldsymbol{\theta})=\mathrm{E}\left[\mathbf{d}_{\boldsymbol{\theta} t}^{\infty}(\boldsymbol{\theta}) \times\right.$ $\left.\mathbf{d}_{\theta t}^{\infty}(\boldsymbol{\theta})^{\prime}\right]$.

(ii) $\frac{1}{\sqrt{T}} \sum_{t=1}^{T}\left\|\mathbf{d}_{\boldsymbol{\theta} t}^{\infty}\left(\boldsymbol{\theta}_{0}\right)-\mathbf{d}_{\boldsymbol{\theta} t}\left(\boldsymbol{\theta}_{0}\right)\right\|=o_{p}(1)$.
(iii) $\sqrt{T} \mathbf{D}_{\boldsymbol{\theta} T}\left(\boldsymbol{\theta}_{0}\right) \stackrel{d}{\rightarrow} N\left(\mathbf{0}, \mathbf{\Omega}_{\boldsymbol{\theta} \boldsymbol{\theta}}\right)$.

\section{Proof.}

(i) We first show that $\boldsymbol{\Omega}_{\boldsymbol{\theta} \boldsymbol{\theta}}=\mathrm{E}\left[\mathbf{d}_{\boldsymbol{\theta} t}^{\infty}\left(\boldsymbol{\theta}_{0}\right) \mathbf{d}_{\boldsymbol{\theta} t}^{\infty}\left(\boldsymbol{\theta}_{0}\right)^{\prime}\right]$ is finite. Denoting $\zeta_{0 t}^{\infty}=$ $\left(\left(\varepsilon_{0}^{2} t / h_{0 t}^{\infty}\right)-1\right)$, we have

$\mathbf{d}_{\boldsymbol{\theta} t}^{\infty}\left(\boldsymbol{\theta}_{0}\right)=\frac{1}{2} \zeta_{0 t}^{\infty} \frac{1}{h_{0 t}^{\infty}} \frac{\partial h_{0 t}^{\infty}}{\partial \boldsymbol{\theta}}+\left[\begin{array}{c}\varepsilon_{0 t} \frac{1}{h_{0 t}^{\infty}} \frac{\partial m_{0 t}}{\partial \boldsymbol{\varphi}} \\ \mathbf{0}\end{array}\right]$,

and it is sufficient to show that $\mathrm{E}\left\|\zeta_{0 t}^{\infty}\left\{\frac{1}{h_{0 t}^{\infty}}\right\}^{2} \frac{\partial h_{0 t}^{\infty}}{\partial \boldsymbol{\theta}} \frac{\partial h_{0 t}^{\infty}}{\partial \boldsymbol{\theta}^{\prime}}\right\|$ and $\mathrm{E} \| \varepsilon_{0 t}^{2}\left\{\frac{1}{h_{0 t}^{\infty}}\right\}^{2} \times$ $\frac{\partial m_{0 t}}{\partial \varphi} \frac{\partial m_{0 t}}{\partial \varphi^{\prime}} \|$ are both finite. Because $h_{0 t}^{\infty} \geq \lambda>0$ for all $t, \xi_{t}=\varepsilon_{0 t} / \sqrt{h_{0 t}^{\infty}}$ and $\mathrm{E}\left[\xi_{t}^{2} \mid \mathcal{F}_{t-1}\right]=1$, this follows immediately from Assumption B3(b) and Proposition 4.

Furthermore, $\Omega_{\theta \boldsymbol{\theta}}$ is positive definite because $\mathrm{E}\left[\zeta_{0 t}^{\infty 2}\right]=k_{c}-1>0$ is independent of $h_{0 t}^{\infty}$ and, by Proposition 2, for any vectors $\mathbf{c}, \mathbf{b}$ of the same dimension of $\boldsymbol{\varphi}$ and $\eta$, respectively: (1) $\mathbf{c}^{\prime} \mathbf{d}_{\boldsymbol{\varphi} t}^{\infty}\left(\boldsymbol{\theta}_{0}\right)=0$, for all $t$ a.s., only if $\mathbf{c}=\mathbf{0}$ and (2) $\mathbf{b}^{\prime} \mathbf{d}_{\boldsymbol{\eta} t}^{\infty}\left(\boldsymbol{\theta}_{0}\right)=0$, for all $t$ a.s., only if $\mathbf{b}=\mathbf{0}$.

(ii) The proof is similar to that of Proposition 5. First, with the preceding notation and $\tilde{\zeta}_{0 t}=\varepsilon_{0 t}^{2} / \tilde{h}_{0 t}-1$, where $\tilde{h}_{0 t}=h_{t}\left(\boldsymbol{\theta}_{0}\right)$, to distinguish it from $h_{0 t}^{\infty}=h_{t}^{\infty}\left(\boldsymbol{\theta}_{0}\right), \mathbf{d}_{\varphi t}^{\infty}\left(\boldsymbol{\theta}_{0}\right)-$ $\mathbf{d}_{\boldsymbol{\varphi} t}\left(\boldsymbol{\theta}_{0}\right)=\frac{1}{2}\left\{\zeta_{0 t}^{\infty} \frac{1}{h_{0 t}^{\infty}} \frac{\partial h_{0 t}^{\infty}}{\partial \boldsymbol{\varphi}}-\tilde{\zeta}_{0 t} \frac{1}{\tilde{h}_{0 t}} \frac{\partial \tilde{h}_{0 t}}{\partial \boldsymbol{\varphi}}\right\}+\left\{\varepsilon_{0 t} \frac{\partial m_{0 t}}{\partial \boldsymbol{\varphi}}\left(\frac{1}{h_{0 t}^{\infty}}-\frac{1}{\tilde{h}_{0 t}}\right)\right\}$, so that

$$
\begin{aligned}
\left\|\frac{1}{\sqrt{T}} \sum_{t=1}^{T}\left(\mathbf{d}_{\boldsymbol{\varphi} t}^{\infty}\left(\boldsymbol{\theta}_{0}\right)-\mathbf{d}_{\varphi t}\left(\boldsymbol{\theta}_{0}\right)\right)\right\| \leq & \frac{1}{2}\left\|\frac{1}{\sqrt{T}} \sum_{t=1}^{T}\left\{\zeta_{0 t}^{\infty} \frac{1}{h_{0 t}^{\infty}} \frac{\partial h_{0 t}^{\infty}}{\partial \boldsymbol{\varphi}}-\tilde{\zeta}_{0 t} \frac{1}{\tilde{h}_{0 t}} \frac{\partial \tilde{h}_{0 t}}{\partial \boldsymbol{\varphi}}\right\}\right\| \\
& +\left\|\frac{1}{\sqrt{T}} \sum_{t=1}^{T}\left\{\varepsilon_{0 t} \frac{\partial m_{0 t}}{\partial \boldsymbol{\varphi}}\left(\frac{1}{h_{0 t}^{\infty}}-\frac{1}{\tilde{h}_{0 t}}\right)\right\}\right\| \\
= & \frac{1}{2}\left\|R_{T}\right\|+\left\|Q_{T}\right\| .
\end{aligned}
$$

It is sufficient to show that $\mathrm{E}\left\|Q_{T}\right\|=o(1)$ and $\mathrm{E}\left\|R_{T}\right\|=o(1)$. By Assumption $\mathrm{A} 3$, and because $h_{t}^{\infty} \geq \alpha_{0}+\psi_{i} a_{t-i}(i \geq 1)$ and $\xi_{t}=\varepsilon_{0 t} / \sqrt{h_{0 t}^{\infty}}$ is $i i d(0,1)$, 


$$
\begin{aligned}
\mathrm{E}\left\|Q_{T}\right\| & \leq \lambda^{-1} \frac{1}{\sqrt{T}} \sum_{t=1}^{T} \mathrm{E}\left\|\xi_{t} \frac{\partial m_{0 t}}{\partial \varphi}\right\|\left|\frac{h_{0 t}^{\infty}-\tilde{h}_{0 t}}{\sqrt{h_{0 t}^{\infty}}}\right| \\
& \leq C \frac{1}{\sqrt{T}} \sum_{t=1}^{T} \mathrm{E}\left[\left\|\xi_{t} \frac{\partial m_{0 t}}{\partial \varphi}\right\|\left\{\sum_{i=t}^{\infty} \frac{\psi_{i 0} a_{0, t-i}}{\sqrt{\alpha_{00}+\psi_{i 0} a_{0, t-i}}}\right\}\right] \\
& \leq K \frac{1}{\sqrt{T}} \sum_{t=1}^{T} \mathrm{E}\left[\left\|\xi_{t} \frac{\partial m_{0 t}}{\partial \varphi}\right\|\left\{\sum_{i=t}^{\infty} \rho^{i}\left|a_{0, t-i}\right|^{1 / 2}\right\}\right]
\end{aligned}
$$

because $0<\alpha_{00}<\infty$ and $x / \sqrt{1+x} \leq \sqrt{x}$, for all $x \geq 0$. Now, by Cauchy-Schwarz inequality, iterative expectations, and Assumption B3(b), E[ $\left[\mid \xi_{t}\left(\partial m_{0 t} / \partial \varphi\right) a_{0, t-i}^{1 / 2} \|\right] \leq$ $\sqrt{\mathrm{E}\left\|\frac{\partial m_{0 t}}{\partial \varphi}\right\|^{2} \mathrm{E}\left|a_{0, t-i}\right|}<\infty$, so that

$\mathrm{E}\left\|Q_{T}\right\| \leq O(1) \frac{1}{\sqrt{T}} \sum_{t=1}^{T} O\left(\rho^{t}\right)=o(1)$.

Next, by (A.14) and $\mathrm{E}\left[\varepsilon_{0 t}^{2} \mid \mathcal{F}_{t-1}\right]=h_{0 t}^{\infty}$,

$\mathrm{E}\left\|R_{T}\right\|$

$$
\begin{aligned}
& \leq \frac{1}{\sqrt{T}} \sum_{t=1}^{T} \mathrm{E}\left\{\varepsilon_{0 t}^{2}\left\|\frac{1}{\left(h_{0 t}^{\infty}\right)^{2}} \frac{\partial h_{0 t}^{\infty}}{\partial \varphi}-\frac{1}{\left(\tilde{h}_{0 t}\right)^{2}} \frac{\partial \tilde{h}_{0 t}}{\partial \varphi}\right\|+\left\|\frac{1}{h_{0 t}^{\infty}} \frac{\partial h_{0 t}^{\infty}}{\partial \varphi}-\frac{1}{\tilde{h}_{0 t}} \frac{\partial \tilde{h}_{0 t}}{\partial \varphi}\right\|\right\} \\
& \leq K \frac{1}{\sqrt{T}} \sum_{t=1}^{T} \mathrm{E}\left\{\frac{1}{h_{0 t}^{\infty}}\left\|\frac{\partial h_{0 t}^{\infty}}{\partial \varphi}-\frac{\partial \tilde{h}_{0 t}}{\partial \varphi}\right\|+\left\|\frac{1}{\tilde{h}_{0 t}} \frac{\partial \tilde{h}_{0 t}}{\partial \varphi}\right\|\left|h_{0 t}^{\infty}-\tilde{h}_{0 t}\right|\right\} .
\end{aligned}
$$

It follows from (A.10), (A.8), (A.2), and arguments similar to Proposition 4 that $\mathrm{E}\left\|R_{T}\right\|=o(1)$.

Second, and in a similar fashion, by (A.5) and (A.7)

$$
\begin{aligned}
& \mathrm{E}\left\|\frac{1}{\sqrt{T}} \sum_{t=1}^{T}\left(\mathbf{d}_{\boldsymbol{\eta} t}^{\infty}\left(\boldsymbol{\theta}_{0}\right)-\mathbf{d}_{\boldsymbol{\eta} t}\left(\boldsymbol{\theta}_{0}\right)\right)\right\| \\
& \quad \leq K \frac{1}{\sqrt{T}} \sum_{t=1}^{T} \mathrm{E}\left\{\frac{1}{h_{0 t}^{\infty}}\left\|\frac{\partial h_{0 t}^{\infty}}{\partial \boldsymbol{\eta}}-\frac{\partial \tilde{h}_{0 t}}{\partial \boldsymbol{\eta}}\right\|+\left\|\frac{1}{\tilde{h}_{0 t}} \frac{\partial \tilde{h}_{0 t}}{\partial \boldsymbol{\eta}}\right\|\left|h_{0 t}^{\infty}-\tilde{h}_{0 t}\right|\right\}=o(1) .
\end{aligned}
$$

Thus $\left\|\frac{1}{\sqrt{T}} \sum_{t=1}^{T}\left(\mathbf{d}_{\boldsymbol{\eta} t}^{\infty}\left(\boldsymbol{\theta}_{0}\right)-\mathbf{d}_{\boldsymbol{\eta} t}\left(\boldsymbol{\theta}_{0}\right)\right)\right\|=o_{p}(1)$, by Markov's inequality.

(iii) As in Lemma 5.2 of Ling and McAleer (2003), a martingale difference central limit theorem (CLT) yields $\sqrt{T} \mathbf{D}_{\boldsymbol{\theta} T}^{\infty}\left(\boldsymbol{\theta}_{0}\right) \stackrel{d}{\rightarrow} N\left(\mathbf{0}, \boldsymbol{\Omega}_{\boldsymbol{\theta} \boldsymbol{\theta}}\right)$ so that (ii) yields $\sqrt{T} \mathbf{D}_{\boldsymbol{\theta} T}\left(\boldsymbol{\theta}_{0}\right) \stackrel{d}{\rightarrow}$ $N\left(\mathbf{0}, \boldsymbol{\Omega}_{\theta \boldsymbol{\theta}}\right)$. 
PROPOSITION 7. Under Assumptions A and B,

(i) $\sup _{\boldsymbol{\theta} \in \boldsymbol{\Theta}}\left\|\mathbf{P}_{\boldsymbol{\theta} \boldsymbol{\theta} T}^{\infty}(\boldsymbol{\theta})-\mathbf{J}_{\boldsymbol{\theta} \boldsymbol{\theta}}(\boldsymbol{\theta})\right\|=o_{p}(1)$, where $\mathbf{J}_{\boldsymbol{\theta} \boldsymbol{\theta}}(\boldsymbol{\theta})=-\mathrm{E}\left[\frac{\partial \mathbf{d}_{\boldsymbol{\theta} t}^{\infty}(\boldsymbol{\theta})}{\partial \boldsymbol{\theta}^{\prime}}\right]$ is finite for all $\boldsymbol{\theta} \in \boldsymbol{\Theta}$ and $\mathbf{J}_{\boldsymbol{\theta} \boldsymbol{\theta}}=\mathbf{J}_{\boldsymbol{\theta} \boldsymbol{\theta}}\left(\boldsymbol{\theta}_{0}\right)$ is positive definite.

(ii) $\sup _{\boldsymbol{\theta} \in \boldsymbol{\Theta}}\left\|\mathbf{P}_{\boldsymbol{\theta} \boldsymbol{\theta} T}^{\infty}(\boldsymbol{\theta})-\mathbf{P}_{\boldsymbol{\theta} \boldsymbol{\theta} T}(\boldsymbol{\theta})\right\|=o_{p}(1)$.

\section{Proof.}

(i) We show that $\mathbf{J}_{\boldsymbol{\theta} \boldsymbol{\theta}}(\boldsymbol{\theta})=-\mathrm{E}\left[\frac{\partial \mathbf{d}_{\boldsymbol{\theta} t}^{\infty}(\boldsymbol{\theta})}{\partial \boldsymbol{\theta}^{\prime}}\right]$ is finite for all $\boldsymbol{\theta} \in \boldsymbol{\Theta}$; it is then straightforward to show that $\mathbf{J}_{\boldsymbol{\theta} \boldsymbol{\theta}}\left(\boldsymbol{\theta}_{0}\right)$ is positive definite. The result then follows from Theorem 3.1 of Ling and McAleer (2003). We have

$$
\begin{aligned}
\frac{\partial \mathbf{d}_{\boldsymbol{\varphi} t}^{\infty}(\boldsymbol{\theta})}{\partial \boldsymbol{\varphi}^{\prime}}= & -\frac{1}{h_{t}^{\infty}} \frac{\partial m_{t}}{\partial \boldsymbol{\varphi}} \frac{\partial m_{t}}{\partial \boldsymbol{\varphi}^{\prime}}-\frac{\varepsilon_{t}}{\left(h_{t}^{\infty}\right)^{2}} \frac{\partial m_{t}}{\partial \boldsymbol{\varphi}} \frac{\partial h_{t}^{\infty}}{\partial \boldsymbol{\varphi}^{\prime}} \\
& -\frac{\varepsilon_{t}}{\left(h_{t}^{\infty}\right)^{2}} \frac{\partial h_{t}^{\infty}}{\partial \boldsymbol{\varphi}} \frac{\partial m_{t}}{\partial \boldsymbol{\varphi}^{\prime}}+\frac{\varepsilon_{t}}{h_{t}^{\infty}} \frac{\partial^{2} m_{t}}{\partial \boldsymbol{\varphi} \partial \boldsymbol{\varphi}^{\prime}} \\
& -\frac{1}{2}\left\{\left(2 \frac{\varepsilon_{t}^{2}}{h_{t}^{\infty}}-1\right) \frac{1}{\left(h_{t}^{\infty}\right)^{2}} \frac{\partial h_{t}^{\infty}}{\partial \boldsymbol{\varphi}} \frac{\partial h_{t}^{\infty}}{\partial \boldsymbol{\varphi}^{\prime}}-\left(\frac{\varepsilon_{t}^{2}}{h_{t}^{\infty}}-1\right)\left(\frac{1}{h_{t}^{\infty}} \frac{\partial^{2} h_{t}^{\infty}}{\partial \boldsymbol{\varphi} \partial \boldsymbol{\varphi}^{\prime}}\right)\right\}, \\
\frac{\partial \mathbf{d}_{\boldsymbol{\eta} t}^{\infty}(\boldsymbol{\theta})}{\partial \boldsymbol{\eta}^{\prime}}= & -\frac{1}{2}\left\{\left(2 \frac{\varepsilon_{t}^{2}}{h_{t}^{\infty}}-1\right) \frac{1}{\left(h_{t}^{\infty}\right)^{2}} \frac{\partial h_{t}^{\infty}}{\partial \boldsymbol{\eta}} \frac{\partial h_{t}^{\infty}}{\partial \boldsymbol{\eta}^{\prime}}-\left(\frac{\varepsilon_{t}^{2}}{h_{t}^{\infty}}-1\right)\left(\frac{1}{h_{t}^{\infty}} \frac{\partial^{2} h_{t}^{\infty}}{\partial \boldsymbol{\eta} \partial \boldsymbol{\eta}^{\prime}}\right)\right\}, \\
\frac{\partial \mathbf{d}_{\boldsymbol{\eta}_{t}}^{\infty}(\boldsymbol{\theta})}{\partial \boldsymbol{\varphi}^{\prime}}= & -\frac{\varepsilon_{t}}{h_{t}^{\infty}} \frac{\partial h_{t}^{\infty}}{\partial \boldsymbol{\eta}} \frac{\partial m_{t}}{\partial \boldsymbol{\varphi}^{\prime}} \\
& -\frac{1}{2}\left\{\left(2 \frac{\varepsilon_{t}^{2}}{h_{t}^{\infty}}-1\right) \frac{1}{\left(h_{t}^{\infty}\right)^{2}} \frac{\partial h_{t}^{\infty}}{\partial \boldsymbol{\eta}} \frac{\partial h_{t}^{\infty}}{\partial \boldsymbol{\varphi}^{\prime}}-\left(\frac{\varepsilon_{t}^{2}}{h_{t}^{\infty}}-1\right)\left(\frac{1}{h_{t}^{\infty}} \frac{\partial^{2} h_{t}^{\infty}}{\partial \boldsymbol{\eta} \partial \boldsymbol{\varphi}^{\prime}}\right)\right\} .
\end{aligned}
$$

Thus, exploiting $h_{t}^{\infty} \geq \lambda>0$ and where $K=\max \left\{\lambda^{-1}, 2 \lambda^{-2}, 1 / 2\right\}$,

$$
\begin{aligned}
& \left\|\frac{\partial \mathbf{d}_{\boldsymbol{~} t}^{\infty}(\boldsymbol{\theta})}{\partial \boldsymbol{\varphi}^{\prime}}\right\| \leq K\left\{\left\|\frac{\partial m_{t}}{\partial \boldsymbol{\varphi}}\right\|^{2}+\left(\varepsilon_{t}^{2}+1\right)\left(\left\|\frac{1}{h_{t}^{\infty}} \frac{\partial h_{t}^{\infty}}{\partial \boldsymbol{\varphi}}\right\|^{2}+\left\|\frac{1}{h_{t}^{\infty}} \frac{\partial^{2} h_{t}^{\infty}}{\partial \boldsymbol{\varphi} \partial \boldsymbol{\varphi}^{\prime}}\right\|\right)\right. \\
& \left.+\left\|\varepsilon_{t} \frac{\partial m_{t}}{\partial \boldsymbol{\varphi}} \frac{1}{h_{t}^{\infty}} \frac{\partial h_{t}^{\infty}}{\partial \varphi^{\prime}}\right\|+\left\|\varepsilon_{t} \frac{\partial^{2} m_{t}}{\partial \boldsymbol{\varphi} \partial \boldsymbol{\varphi}^{\prime}}\right\|\right\} .
\end{aligned}
$$

By Assumption B3(b), Proposition 4(i) and (ii), and Cauchy-Schwarz inequality, $\operatorname{Esup}_{\boldsymbol{\theta}}\left\|\frac{\partial \mathbf{d}_{\varphi t}^{\infty}(\boldsymbol{\theta})}{\partial \boldsymbol{\varphi}^{\prime}}\right\|<\infty$. 
By similar arguments,

$$
\begin{gathered}
\operatorname{Eup}_{\boldsymbol{\theta}}\left\|\frac{\partial \mathbf{d}_{\boldsymbol{\eta} t}^{\infty}(\boldsymbol{\theta})}{\partial \boldsymbol{\eta}^{\prime}}\right\| \leq K E \sup _{\boldsymbol{\theta}}\left\{\left(\varepsilon_{t}^{2}+1\right)\left(\left\|\frac{1}{h_{t}^{\infty}} \frac{\partial h_{t}^{\infty}}{\partial \boldsymbol{\eta}}\right\|^{2}+\left\|\frac{1}{h_{t}^{\infty}} \frac{\partial^{2} h_{t}^{\infty}}{\partial \boldsymbol{\eta} \partial \boldsymbol{\eta}^{\prime}}\right\|\right)\right\}<\infty, \\
\operatorname{Eup}_{\boldsymbol{\theta}}\left\|\frac{\partial \mathbf{d}_{\boldsymbol{\eta} t}^{\infty}(\boldsymbol{\theta})}{\partial \boldsymbol{\varphi}^{\prime}}\right\| \leq C\left\{\left(\varepsilon_{t}^{2}+1\right)\left(\left\|\frac{1}{h_{t}^{\infty}} \frac{\partial h_{t}^{\infty}}{\partial \boldsymbol{\eta}} \frac{1}{h_{t}^{\infty}} \frac{\partial h_{t}^{\infty}}{\partial \boldsymbol{\varphi}^{\prime}}\right\|+\left\|\frac{1}{h_{t}^{\infty}} \frac{\partial^{2} h_{t}^{\infty}}{\partial \boldsymbol{\eta} \partial \boldsymbol{\varphi}^{\prime}}\right\|\right)\right. \\
\left.+\left\|\varepsilon_{t} \frac{1}{h_{t}^{\infty}} \frac{\partial h_{t}^{\infty}}{\partial \boldsymbol{\eta}} \frac{\partial m_{t}}{\partial \boldsymbol{\varphi}^{\prime}}\right\|\right\}<\infty .
\end{gathered}
$$

(ii) Note that $\sup _{\boldsymbol{\theta}}\left\|\mathbf{P}_{\boldsymbol{\theta} \boldsymbol{\theta} T}^{\infty}(\boldsymbol{\theta})-\mathbf{P}_{\boldsymbol{\theta} \boldsymbol{\theta} T}(\boldsymbol{\theta})\right\| \leq T^{-1} \sum_{t} \sup _{\boldsymbol{\theta}} \|\left(\partial^{2} l_{t}^{\infty} / \partial \boldsymbol{\theta} \partial \boldsymbol{\theta}^{\prime}\right)-$ $\left(\partial^{2} l_{t} / \partial \boldsymbol{\theta} \partial \boldsymbol{\theta}^{\prime}\right) \|$, and we consider the latter. First,

$$
\begin{aligned}
& \left\|\frac{\partial \mathbf{d}_{\boldsymbol{\varphi} t}^{\infty}(\boldsymbol{\theta})}{\partial \boldsymbol{\varphi}^{\prime}}-\frac{\partial \mathbf{d}_{\boldsymbol{~} t}(\boldsymbol{\theta})}{\partial \boldsymbol{\varphi}^{\prime}}\right\| \leq\left\{\left\|\frac{\partial m_{t}}{\partial \boldsymbol{\varphi}}\right\|^{2}+\left\|\varepsilon_{t} \frac{\partial^{2} m_{t}}{\partial \boldsymbol{\varphi} \partial \boldsymbol{\varphi}^{\prime}}\right\|\right\}\left|\frac{1}{h_{t}^{\infty}}-\frac{1}{h_{t}}\right| \\
& +2\left\|\varepsilon_{t} \frac{\partial m_{t}}{\partial \boldsymbol{\varphi}}\right\|\left\|\frac{1}{\left(h_{t}^{\infty}\right)^{2}} \frac{\partial h_{t}^{\infty}}{\partial \varphi}-\frac{1}{h_{t}^{2}} \frac{\partial h_{t}}{\partial \boldsymbol{\varphi}}\right\| \\
& +\varepsilon_{t}^{2}\left\|\frac{1}{\left(h_{t}^{\infty}\right)^{3}} \frac{\partial h_{t}^{\infty}}{\partial \varphi} \frac{\partial h_{t}^{\infty}}{\partial \varphi^{\prime}}-\frac{1}{h_{t}^{3}} \frac{\partial h_{t}}{\partial \varphi} \frac{\partial h_{t}}{\partial \varphi^{\prime}}\right\| \\
& +\frac{1}{2}\left\|\frac{1}{\left(h_{t}^{\infty}\right)^{2}} \frac{\partial h_{t}^{\infty}}{\partial \varphi} \frac{\partial h_{t}^{\infty}}{\partial \varphi^{\prime}}-\frac{1}{h_{t}^{2}} \frac{\partial h_{t}}{\partial \varphi} \frac{\partial h_{t}}{\partial \varphi^{\prime}}\right\| \\
& +\frac{1}{2} \varepsilon_{t}^{2}\left\|\frac{1}{\left(h_{t}^{\infty}\right)^{2}} \frac{\partial^{2} h_{t}^{\infty}}{\partial \varphi \partial \varphi^{\prime}}-\frac{1}{h_{t}^{2}} \frac{\partial^{2} h_{t}}{\partial \varphi \partial \varphi^{\prime}}\right\| \\
& +\frac{1}{2}\left\|\frac{1}{h_{t}^{\infty}} \frac{\partial^{2} h_{t}^{\infty}}{\partial \boldsymbol{\varphi} \partial \varphi^{\prime}}-\frac{1}{h_{t}} \frac{\partial^{2} h_{t}}{\partial \varphi \partial \varphi^{\prime}}\right\| \\
& =\sum_{j=1}^{6}\left\|R_{j t}\right\|
\end{aligned}
$$

Consider $1 / T \sum_{t=1}^{T} \sup _{\boldsymbol{\theta} \in \Theta}\left\|R_{1 t}\right\|$, where $R_{1 t}=X_{t} u_{t}$, with $u_{t}=\sup _{\theta}\left|\left(1 / h_{t}^{\infty}\right)-\left(1 / h_{t}\right)\right|$, and apply Lemma 4.5 of Ling and McAleer (2003). We know that $u_{t}<2 \lambda^{-1}$ and $T^{-1}$ $\sum_{t=1}^{T} u_{t}=o_{p}(1)$, and because $\operatorname{Esup}_{\boldsymbol{\theta}}\left\{\left\|\frac{\partial m_{t}}{\partial \boldsymbol{\varphi}}\right\|^{2}+\left\|\varepsilon_{t} \frac{\partial^{2} m_{t}}{\partial \varphi \varphi^{\prime}}\right\|\right\}<\infty$, by Assumptions B3 (b) and (c), we have $1 / T \sum_{t=1}^{T} \sup _{\boldsymbol{\theta} \in \Theta}\left\|R_{1 t}\right\|=o_{p}(1)$. By Proposition 4, $\operatorname{Esup}_{\boldsymbol{\theta}}\left\|R_{j t}\right\|=$ $O\left(\rho^{t}\right), j=2, \ldots, 6$, so that $1 / T \sum_{t=1}^{T} \sup _{\boldsymbol{\theta} \in \Theta}\left\|R_{j t}\right\|=o_{p}(1)$, by Markov's inequality. 
Similarly, $\sup _{\boldsymbol{\theta} \in \Theta} \frac{1}{T} \sum_{t=1}^{T}\left\|\left(\partial \mathbf{d}_{\eta t}^{\infty}(\theta) / \partial \boldsymbol{\varphi}^{\prime}\right)-\left(\partial \mathbf{d}_{\eta t}(\theta) / \partial \boldsymbol{\varphi}^{\prime}\right)\right\|=o_{p}(1)$, because

$$
\begin{aligned}
\left\|\frac{\partial \mathbf{d}_{\boldsymbol{\eta}}^{\infty}(\boldsymbol{\theta})}{\partial \boldsymbol{\varphi}^{\prime}}-\frac{\partial \mathbf{d}_{\boldsymbol{\eta} t}(\boldsymbol{\theta})}{\partial \boldsymbol{\varphi}^{\prime}}\right\| \leq & \varepsilon_{t}^{2}\left\|\frac{1}{\left(h_{t}^{\infty}\right)^{3}} \frac{\partial h_{t}^{\infty}}{\partial \boldsymbol{\eta}} \frac{\partial h_{t}^{\infty}}{\partial \boldsymbol{\varphi}^{\prime}}-\frac{1}{h_{t}^{3}} \frac{\partial h_{t}}{\partial \boldsymbol{\eta}} \frac{\partial h_{t}}{\partial \boldsymbol{\varphi}^{\prime}}\right\| \\
& +\frac{1}{2}\left\|\frac{1}{\left(h_{t}^{\infty}\right)^{2}} \frac{\partial h_{t}^{\infty}}{\partial \boldsymbol{\eta}} \frac{\partial h_{t}^{\infty}}{\partial \boldsymbol{\varphi}^{\prime}}-\frac{1}{h_{t}^{2}} \frac{\partial h_{t}}{\partial \boldsymbol{\eta}} \frac{\partial h_{t}}{\partial \boldsymbol{\varphi}^{\prime}}\right\| \\
& +\frac{1}{2} \varepsilon_{t}^{2}\left\|\frac{1}{\left(h_{t}^{\infty}\right)^{2}} \frac{\partial^{2} h_{t}^{\infty}}{\partial \boldsymbol{\eta} \partial \boldsymbol{\varphi}^{\prime}}-\frac{1}{h_{t}^{2}} \frac{\partial^{2} h_{t}}{\partial \boldsymbol{\eta} \partial \boldsymbol{\varphi}^{\prime}}\right\| \\
& +\frac{1}{2}\left\|\frac{1}{h_{t}^{\infty}} \frac{\partial^{2} h_{t}^{\infty}}{\partial \boldsymbol{\eta} \partial \boldsymbol{\varphi}^{\prime}}-\frac{1}{h_{t}} \frac{\partial^{2} h_{t}}{\partial \boldsymbol{\eta} \partial \boldsymbol{\varphi}^{\prime}}\right\| \\
& +2\left\|\varepsilon_{t} \frac{\partial m_{t}}{\partial \boldsymbol{\varphi}}\right\|\left\|\frac{1}{h_{t}^{\infty}} \frac{\partial h_{t}^{\infty}}{\partial \boldsymbol{\eta}}-\frac{1}{h_{t}} \frac{\partial h_{t}}{\partial \boldsymbol{\eta}}\right\| \\
= & K \sum_{j=1}^{5}\left\|R_{j t}\right\|,
\end{aligned}
$$

and by Proposition $4 \operatorname{Esup}_{\boldsymbol{\theta}}\left\|R_{j t}\right\|=O\left(\rho^{t}\right), j=1, \ldots, 5$.

Finally, and analogously,

$$
\begin{aligned}
\left\|\frac{\partial \mathbf{d}_{\boldsymbol{\eta} t}^{\infty}(\boldsymbol{\theta})}{\partial \boldsymbol{\eta}^{\prime}}-\frac{\partial \mathbf{d}_{\boldsymbol{\eta} t}(\boldsymbol{\theta})}{\partial \boldsymbol{\eta}^{\prime}}\right\| \leq & \varepsilon_{t}^{2}\left\|\frac{1}{\left(h_{t}^{\infty}\right)^{3}} \frac{\partial h_{t}^{\infty}}{\partial \boldsymbol{\eta}} \frac{\partial h_{t}^{\infty}}{\partial \boldsymbol{\eta}^{\prime}}-\frac{1}{h_{t}^{3}} \frac{\partial h_{t}}{\partial \boldsymbol{\eta}} \frac{\partial h_{t}}{\partial \boldsymbol{\eta}^{\prime}}\right\| \\
& +\frac{1}{2}\left\|\frac{1}{\left(h_{t}^{\infty}\right)^{2}} \frac{\partial h_{t}^{\infty}}{\partial \boldsymbol{\eta}} \frac{\partial h_{t}^{\infty}}{\partial \boldsymbol{\eta}^{\prime}}-\frac{1}{h_{t}^{2}} \frac{\partial h_{t}}{\partial \boldsymbol{\eta}} \frac{\partial h_{t}}{\partial \boldsymbol{\eta}^{\prime}}\right\| \\
& +\frac{1}{2} \varepsilon_{t}^{2}\left\|\frac{1}{\left(h_{t}^{\infty}\right)^{2}} \frac{\partial^{2} h_{t}^{\infty}}{\partial \boldsymbol{\eta} \partial \boldsymbol{\eta}^{\prime}}-\frac{1}{h_{t}^{2}} \frac{\partial^{2} h_{t}}{\partial \boldsymbol{\eta} \partial \boldsymbol{\eta}^{\prime}}\right\| \\
& +\frac{1}{2}\left\|\frac{1}{h_{t}^{\infty}} \frac{\partial^{2} h_{t}^{\infty}}{\partial \boldsymbol{\eta} \partial \boldsymbol{\eta}^{\prime}}-\frac{1}{h_{t}} \frac{\partial^{2} h_{t}}{\partial \boldsymbol{\eta} \partial \boldsymbol{\eta}^{\prime}}\right\|
\end{aligned}
$$

so that, by Proposition 4, $\operatorname{Esup}_{\boldsymbol{\theta}}\left\|\left(\partial \mathbf{d}_{\boldsymbol{\eta} t}^{\infty}(\boldsymbol{\theta}) / \partial \boldsymbol{\eta}^{\prime}\right)-\left(\partial \mathbf{d}_{\boldsymbol{\eta} t}(\boldsymbol{\theta}) / \partial \boldsymbol{\eta}^{\prime}\right)\right\|=O\left(\rho^{t}\right)$.

This completes the proof.

Proof of Theorem 1. By Proposition 5, and as in Ling and McAleer (2003), $\hat{\boldsymbol{\theta}}=\arg \max _{\boldsymbol{\theta}} L_{T}(\boldsymbol{\theta})$ is consistent. The limit distribution then follows from a standard mean value expansion of $\mathbf{D}_{\boldsymbol{\theta} T}(\hat{\boldsymbol{\theta}})=\mathbf{0}$, exploiting Propositions 6 and 7, as follows. First, $\mathbf{0}=\sqrt{T} \mathbf{D}_{\boldsymbol{\theta} T}\left(\boldsymbol{\theta}_{0}\right)-\mathbf{P}_{\boldsymbol{\theta} \boldsymbol{\theta} T}(\tilde{\boldsymbol{\theta}}) \sqrt{T}\left(\hat{\boldsymbol{\theta}}-\boldsymbol{\theta}_{0}\right)$, where $\tilde{\boldsymbol{\theta}}$ is the usual "mean value" satisfying $\tilde{\boldsymbol{\theta}}=\boldsymbol{\theta}_{0}+o_{p}(1)$. By Propositions 6 and $7, \sqrt{T} \mathbf{D}_{\boldsymbol{\theta} T}\left(\boldsymbol{\theta}_{0}\right)=O_{p}(1)$ and $\mathbf{P}_{\boldsymbol{\theta} \boldsymbol{\theta} T}(\tilde{\boldsymbol{\theta}})=$ $O_{p}(1)$, so that $\sqrt{T}\left(\hat{\boldsymbol{\theta}}-\boldsymbol{\theta}_{0}\right)=O_{p}(1)$. Second, by Proposition 7 and the triangle inequality, $\mathbf{P}_{\boldsymbol{\theta} \boldsymbol{\theta} T}(\tilde{\boldsymbol{\theta}})=\mathbf{J}_{\boldsymbol{\theta} \boldsymbol{\theta}}+o_{p}(1)$. Third, because $\mathbf{J}_{\boldsymbol{\theta} \boldsymbol{\theta}}$ is positive definite, $\sqrt{T}\left(\hat{\boldsymbol{\theta}}-\boldsymbol{\theta}_{0}\right)=$ 
$\mathbf{J}_{\boldsymbol{\theta} \boldsymbol{\theta}}^{-1} \sqrt{T} \mathbf{D}_{\boldsymbol{\theta} T}\left(\boldsymbol{\theta}_{0}\right)+o_{p}(1)$, and the result follows from Proposition 6. Finally, the expressions for $\boldsymbol{\Omega}_{\boldsymbol{\theta} \boldsymbol{\theta}}$ and $\mathbf{J}_{\boldsymbol{\theta} \boldsymbol{\theta}}$ are also easily obtained from the previous results in Propositions 6 and 7.

Proof of Lemma 1. The proof follows from previous results. We know from these results and/or assumptions made that $\operatorname{Esup}_{\boldsymbol{\theta}}\left\|\mathbf{q}_{t}^{\infty} \mathbf{q}_{t}^{\infty \prime}\right\|<\infty$, for $\mathbf{q}_{t}^{\infty \prime}=\left(\left(1 / \sqrt{h_{t}^{\infty}}\right) \mathbf{f}_{t}^{\prime}\right.$, $\left.\mathbf{c}_{t}^{\infty \prime}, \mathbf{x}_{t}^{\infty \prime}\right)$. Moreover, $T^{-1} \sum_{t=1}^{T} \sup _{\boldsymbol{\theta}}\left\|\mathbf{q}_{t}^{\infty} \mathbf{q}_{t}^{\infty \prime}-\mathbf{q}_{t} \mathbf{q}_{t}^{\prime}\right\|=o_{p}(1)$ because

$T^{-1} \sum_{t=1}^{T}\left\|\mathbf{q}_{t}^{\infty} \mathbf{q}_{t}^{\infty \prime}-\mathbf{q}_{t} \mathbf{q}_{t}^{\prime}\right\| \leq 2 T^{-1} \sum_{t=1}^{T}\left\|\mathbf{q}_{t}^{\infty}-\mathbf{q}_{t}\right\|\left\|\mathbf{q}_{t}^{\infty}\right\|+T^{-1} \sum_{t=1}^{T}\left\|\mathbf{q}_{t}^{\infty}-\mathbf{q}_{t}\right\|^{2}$.

It is readily shown, using Proposition 4 and related results, that $\frac{1}{\sqrt{T}} \sum_{t=1}^{T} \sup _{\boldsymbol{\theta}} \| \mathbf{q}_{t}^{\infty}-$ $\mathbf{q}_{t} \|=o_{p}(1)$, so that $T^{-1} \sum_{t=1}^{T} \sup _{\boldsymbol{\theta}}\left\|\mathbf{q}_{t}^{\infty}-\mathbf{q}_{t}\right\|^{2}=o_{p}(1)$ (because $\frac{1}{T} \sum_{t=1}^{T} z_{t}^{2} \leq$ $\left\{\frac{1}{\sqrt{T}} \sum_{t=1}^{T} z_{t}\right\}^{2}$, when $z_{t} \geq 0$ for all $\left.t\right)$. In addition,

$T^{-1} \sum_{t=1}^{T} \sup _{\boldsymbol{\theta}}\left\|\mathbf{q}_{t}^{\infty}-\mathbf{q}_{t}\right\|\left\|\mathbf{q}_{t}^{\infty}\right\| \leq \sqrt{T^{-1} \sum_{t=1}^{T} \sup _{\boldsymbol{\theta}}\left\|\mathbf{q}_{t}^{\infty}-\mathbf{q}_{t}\right\|^{2} T^{-1} \sum_{t=1}^{T} \sup _{\boldsymbol{\theta}}\left\|\mathbf{q}_{t}^{\infty}\right\|^{2}}=o_{p}(1)$ because $T^{-1} \sum_{t=1}^{T} \sup _{\boldsymbol{\theta}}\left\|\mathbf{q}_{t}^{\infty}\right\|^{2}=O_{p}(1)$. Therefore, by a ULLN and the triangle inequality, $T^{-1} \sum_{t=1}^{T}\left(\mathbf{q}_{t} \mathbf{q}_{t}^{\prime}\right)_{\boldsymbol{\theta}=\hat{\boldsymbol{\theta}}}-\mathrm{E}\left[\mathbf{q}_{t}^{\infty} \mathbf{q}_{t}^{\infty \prime}\right]_{\boldsymbol{\theta}=\boldsymbol{\theta}_{0}}=o_{p}(1)$.

We also need to show that $\hat{k}_{c}=k_{c}+o_{p}(1)$ and $\hat{v}_{c}=v_{c}+o_{p}(1)$. By similar arguments, $\operatorname{Esup}_{\boldsymbol{\theta}}\left(\left(\varepsilon_{t}^{2} / h_{t}^{\infty}\right)-1\right)^{2}<\infty$ and $\frac{1}{T} \sum_{t=1}^{T} \sup _{\boldsymbol{\theta}}\left\|\left(\frac{\varepsilon_{t}^{2}}{h_{t}^{\infty}}-1\right)^{2}-\left(\frac{\varepsilon_{t}^{2}}{h_{t}}-1\right)^{2}\right\| \leq K \frac{1}{T} \sum_{t=1}^{T} \sup _{\boldsymbol{\theta}}\left|\varepsilon_{t}^{4}-\varepsilon_{t}^{2}\right|\left|\frac{1}{h_{t}^{\infty}}-\frac{1}{h_{t}}\right|=o_{p}(1)$ by (A.4) and Lemma 4.5 of Ling and McAleer (2003). Finally, Esup $\boldsymbol{\theta}\left(\frac{\varepsilon_{t}}{\sqrt{h_{t}^{\infty}}}\right)^{3}<\infty$, and by exactly the same reasoning $\frac{1}{T} \sum_{t=1}^{T} \sup _{\boldsymbol{\theta}}\left\|\left(\frac{\varepsilon_{t}}{\sqrt{h_{t}^{\infty}}}\right)^{3}-\left(\frac{\varepsilon_{t}}{\sqrt{h_{t}}}\right)^{3}\right\| \leq \frac{1}{T} \sum_{t=1}^{T} \sup _{\boldsymbol{\theta}}\left|\varepsilon_{t}^{3}\right|\left|\frac{1}{h_{t}^{\infty 3 / 2}}-\frac{1}{h_{t}^{3 / 2}}\right|=o_{p}(1)$, because $\left|\left(1 / h_{t}^{\infty 3 / 2}\right)-\left(1 / h_{t}^{3 / 2}\right)\right| \leq K\left|h_{t}^{\infty}-h_{t}\right|$.

\section{APPENDIX B}

Proof of Theorem 2. We shall establish the following results:

(a) $\boldsymbol{\Omega}=\mathrm{E}\left[\mathbf{d}_{t}^{\infty}\left(\boldsymbol{\theta}_{0}\right) \mathbf{d}_{t}^{\infty}\left(\boldsymbol{\theta}_{0}\right)^{\prime}\right]$ is finite and positive definite, where $\mathbf{d}_{t}^{\infty}(\boldsymbol{\theta})^{\prime}=\left(\mathbf{d}_{\boldsymbol{\varphi} t}^{\infty}(\boldsymbol{\theta})^{\prime}\right.$, $\left.\mathbf{d}_{\eta t}^{\infty}(\boldsymbol{\theta})^{\prime}, \mathbf{d}_{\pi t}^{\infty}(\boldsymbol{\theta})^{\prime}\right)$ 
(b) $\frac{1}{\sqrt{T}} \sum_{t=1}^{T} \sup _{\boldsymbol{\theta}}\left\|\mathbf{d}_{\boldsymbol{\pi} t}^{\infty}(\boldsymbol{\theta})-\mathbf{d}_{\boldsymbol{\pi} t}(\boldsymbol{\theta})\right\|=o_{p}(1)$;

(c) $\mathbf{J}_{\boldsymbol{\pi} \boldsymbol{\theta}}(\boldsymbol{\theta})$ is finite for all $\boldsymbol{\theta} \in \boldsymbol{\Theta}$, so that $\sup _{\boldsymbol{\theta}}\left\|\mathbf{P}_{\boldsymbol{\pi} \boldsymbol{\theta} T}^{\infty}(\boldsymbol{\theta})-\mathbf{J}_{\boldsymbol{\pi} \boldsymbol{\theta}}(\boldsymbol{\theta})\right\|=o_{p}$ (1), where $\mathbf{P}_{\boldsymbol{\pi} \boldsymbol{\theta} T}^{\infty}(\boldsymbol{\theta})=\frac{1}{T} \sum_{t=1}^{T} \frac{\partial \mathbf{d}_{\boldsymbol{\pi} t}^{\infty}(\boldsymbol{\theta})}{\partial \boldsymbol{\theta}^{\prime}}$.

Given (a) and similarly to Proposition $6, \sqrt{T} \mathbf{D}_{T}^{\infty}\left(\boldsymbol{\theta}_{0}\right) \stackrel{d}{\rightarrow} N(\mathbf{0}, \boldsymbol{\Omega})$, where $\mathbf{D}_{T}^{\infty}(\boldsymbol{\theta})=$ $T^{-1} \sum \mathbf{d}_{t}^{\infty}(\boldsymbol{\theta})$ and $\boldsymbol{\Omega}$ is positive definite provided $\mathbf{r}_{t}^{\infty}$ does not contain redundant terms (e.g., linear combinations of $\mathbf{c}_{t}^{\infty}$ and/or $\mathbf{x}_{t}^{\infty}$ ). By (b)

$\sup _{\boldsymbol{\theta}}\left\|\sqrt{T} \mathbf{D}_{\boldsymbol{\pi} T}^{\infty}(\boldsymbol{\theta})-\sqrt{T} \mathbf{D}_{\boldsymbol{\pi} T}(\boldsymbol{\theta})\right\| \leq \frac{1}{\sqrt{T}} \sum_{t=1}^{T} \sup _{\boldsymbol{\theta}}\left\|\mathbf{d}_{\boldsymbol{\pi} t}^{\infty}(\boldsymbol{\theta})-\mathbf{d}_{\boldsymbol{\pi} t}(\boldsymbol{\theta})\right\|=o_{p}(1)$,

so that $\sqrt{T} \mathbf{D}_{\boldsymbol{\pi} T}(\hat{\boldsymbol{\theta}})=\sqrt{T} \mathbf{D}_{\boldsymbol{\pi} T}^{\infty}(\hat{\boldsymbol{\theta}})+o_{p}(1)$ and we can deal with $\sqrt{T} \mathbf{D}_{\boldsymbol{\pi} T}^{\infty}(\hat{\boldsymbol{\theta}})$. A mean value expansion of $\sqrt{T} \mathbf{D}_{\pi T}^{\infty}(\hat{\boldsymbol{\theta}})$ about $\hat{\boldsymbol{\theta}}=\boldsymbol{\theta}_{0}$ yields

$\sqrt{T} \mathbf{D}_{\boldsymbol{\pi} T}^{\infty}(\hat{\boldsymbol{\theta}})=\sqrt{T} \mathbf{D}_{\boldsymbol{\pi} T}^{\infty}\left(\boldsymbol{\theta}_{0}\right)-\mathbf{P}_{\boldsymbol{\pi} \boldsymbol{\theta} T}^{\infty}(\tilde{\boldsymbol{\theta}}) \sqrt{T}\left(\hat{\boldsymbol{\theta}}-\boldsymbol{\theta}_{0}\right)$

where $\tilde{\boldsymbol{\theta}}$ is the usual "mean value" satisfying $\tilde{\boldsymbol{\theta}}=\boldsymbol{\theta}_{0}+o_{p}(1)$. Because $\hat{\boldsymbol{\theta}}$ is consistent for $\boldsymbol{\theta}_{0}$, the triangle inequality and (c) ensure that $\mathbf{P}_{\boldsymbol{\pi} \boldsymbol{\theta} T}^{\infty}(\tilde{\boldsymbol{\theta}})=\mathbf{J}_{\boldsymbol{\pi} \boldsymbol{\theta}}+o_{p}(1)$, and, substituting $\sqrt{T}\left(\hat{\boldsymbol{\theta}}-\boldsymbol{\theta}_{0}\right)=\mathbf{J}_{\boldsymbol{\theta} \boldsymbol{\theta}}^{-1} \sqrt{T} \mathbf{D}_{\boldsymbol{\theta} T}^{\infty}\left(\boldsymbol{\theta}_{0}\right)+o_{p}(1)$ from Theorem 1, yields

$$
\begin{aligned}
\sqrt{T} \mathbf{D}_{\boldsymbol{\pi} T}^{\infty}(\hat{\boldsymbol{\theta}}) & =\sqrt{T} \mathbf{D}_{\boldsymbol{\pi} T}^{\infty}\left(\boldsymbol{\theta}_{0}\right)-\mathbf{J}_{\boldsymbol{\pi} \boldsymbol{\theta}} \mathbf{J}_{\boldsymbol{\theta} \boldsymbol{\theta}}^{-1} \sqrt{T} \mathbf{D}_{\boldsymbol{\theta} T}^{\infty}\left(\boldsymbol{\theta}_{0}\right)+o_{p}(1) \\
& =\mathbf{A} \sqrt{T} \mathbf{D}_{T}^{\infty}\left(\boldsymbol{\theta}_{0}\right)+o_{p}(1),
\end{aligned}
$$

and the result follows.

For the particular class of tests characterized by the test indicator (9),

$$
\begin{aligned}
\frac{\partial \mathbf{d}_{\boldsymbol{\pi} t}^{\infty}(\boldsymbol{\theta})}{\partial \boldsymbol{\varphi}^{\prime}} & =-2 \frac{\varepsilon_{t}}{h_{t}^{\infty}} \frac{\partial m_{t}}{\partial \boldsymbol{\varphi}^{\prime}} \mathbf{r}_{t}^{\infty}-\frac{\varepsilon_{t}^{2}}{h_{t}^{\infty}} \mathbf{r}_{t}^{\infty}\left(\frac{1}{h_{t}^{\infty}} \frac{\partial h_{t}^{\infty}}{\partial \boldsymbol{\varphi}^{\prime}}\right)+\left(\frac{\varepsilon_{t}^{2}}{h_{t}^{\infty}}-1\right) \frac{\partial \mathbf{r}_{t}^{\infty}}{\partial \boldsymbol{\varphi}^{\prime}} \\
\frac{\partial \mathbf{d}_{\boldsymbol{\pi} t}^{\infty}(\boldsymbol{\theta})}{\partial \boldsymbol{\eta}^{\prime}} & =-\frac{\varepsilon_{t}^{2}}{h_{t}^{\infty}} \mathbf{r}_{t}^{\infty}\left(\frac{1}{h_{t}^{\infty}} \frac{\partial h_{t}^{\infty}}{\partial \boldsymbol{\eta}^{\prime}}\right)+\left(\frac{\varepsilon_{t}^{2}}{h_{t}^{\infty}}-1\right) \frac{\partial \mathbf{r}_{t}^{\infty}}{\partial \boldsymbol{\eta}^{\prime}}
\end{aligned}
$$

so that $\mathbf{J}_{\boldsymbol{\pi} \varphi}=\mathrm{E}\left[\mathbf{r}_{t}^{\infty} \mathbf{c}_{t}^{\infty \prime}\right]_{\boldsymbol{\theta}=\boldsymbol{\theta}_{0}}$ and $\mathbf{J}_{\boldsymbol{\pi} \boldsymbol{\eta}}=\mathrm{E}\left[\mathbf{r}_{t}^{\infty} \mathbf{x}_{t}^{\infty \prime}\right]_{\boldsymbol{\theta}=\boldsymbol{\theta}_{0}}$ and similarly, from expressions for $\mathbf{d}_{\boldsymbol{\theta} t}^{\infty}\left(\boldsymbol{\theta}_{0}\right)$ in the proof of Proposition 6 and $\mathbf{d}_{\boldsymbol{\pi} t}^{\infty}\left(\boldsymbol{\theta}_{0}\right)=\zeta_{0 t}^{\infty} \mathbf{r}_{0 t}^{\infty}$, where $\zeta_{0 t}^{\infty}=\varepsilon_{0 t}^{2} / h_{0 t}^{\infty}-1$,

$$
\begin{aligned}
& \boldsymbol{\Omega}_{\pi \boldsymbol{\varphi}}=v_{c} \mathrm{E}\left[\frac{1}{\sqrt{h_{t}^{\infty}}} \mathbf{r}_{t}^{\infty} \mathbf{f}_{t}^{\prime}\right]_{\boldsymbol{\theta}=\boldsymbol{\theta}_{0}}+\frac{\left(k_{c}-1\right)}{2} \mathrm{E}\left[\mathbf{r}_{t}^{\infty} \mathbf{c}_{t}^{\infty \prime}\right]_{\boldsymbol{\theta}=\boldsymbol{\theta}_{0}}, \\
& \boldsymbol{\Omega}_{\boldsymbol{\pi} \boldsymbol{\eta}}=\frac{\left(k_{c}-1\right)}{2} \mathrm{E}\left[\mathbf{r}_{t}^{\infty} \mathbf{x}_{t}^{\infty \prime}\right]_{\boldsymbol{\theta}=\boldsymbol{\theta}_{0}}, \\
& \mathbf{\Omega}_{\boldsymbol{\pi} \boldsymbol{\pi}}=\left(k_{c}-1\right) \mathrm{E}\left[\mathbf{r}_{t}^{\infty} \mathbf{r}_{t}^{\infty \prime}\right]_{\boldsymbol{\theta}=\boldsymbol{\theta}_{0}} .
\end{aligned}
$$

We now establish that (a)-(c) hold: 
(a) Because $\boldsymbol{\Omega}_{\boldsymbol{\theta} \boldsymbol{\theta}}$ is finite (Proposition 6), by Cauchy-Schwarz inequality we only have to show that $\mathrm{E}\left[\mathbf{d}_{\boldsymbol{\pi} t}^{\infty}\left(\boldsymbol{\theta}_{0}\right) \mathbf{d}_{\boldsymbol{\pi} t}^{\infty}\left(\boldsymbol{\theta}_{0}\right)^{\prime}\right]$ is finite. The latter is true because

$\underset{\boldsymbol{\theta}}{\mathrm{Esup}}\left\|\mathbf{r}_{t}^{\infty}\right\|^{2}<\infty$, so that $\mathrm{E}\left\|\left(\zeta_{0 t}^{\infty}\right)^{2} \mathbf{r}_{0 t}^{\infty} \mathbf{r}_{0 t}^{\infty \prime}\right\|=\left(k_{c}-1\right) \mathrm{E}\left\|\mathbf{r}_{0 t}^{\infty}\right\|^{2}<\infty$.

(b) It can be shown that

$$
\begin{aligned}
\left\|\mathbf{d}_{\boldsymbol{\pi} t}^{\infty}(\boldsymbol{\theta})-\mathbf{d}_{\boldsymbol{\pi} t}(\boldsymbol{\theta})\right\| \leq K & \left\{\left|\varepsilon_{t}^{2}+1\right|\left\|\mathbf{r}_{t}^{\infty}-\mathbf{r}_{t}\right\|+\varepsilon_{t}^{2}\left\|\mathbf{r}_{t}^{\infty}\right\|\left|\frac{h_{t}^{\infty}-h_{t}}{h_{t}^{\infty}}\right|\right. \\
& \left.+\varepsilon_{t}^{2}\left\|\mathbf{r}_{t}^{\infty}-\mathbf{r}_{t}\right\|\left|\frac{h_{t}^{\infty}-h_{t}}{h_{t}^{\infty}}\right|\right\} \\
=K \sum_{j=1}^{3} R_{j t} . &
\end{aligned}
$$

By assumption, $\frac{1}{\sqrt{T}} \sum_{t=1}^{T} \operatorname{Esup}_{\boldsymbol{\theta}} R_{1 t}=o(1)$.

By (A.3) and because $\operatorname{Esup}_{\theta}\left\|\varepsilon_{t}^{2} \mathbf{r}_{t}^{\infty} \varepsilon_{t-i}^{2 s}\right\| \leq \sqrt{\operatorname{Esup}_{\boldsymbol{\theta}}\left\|\mathbf{r}_{t}^{\infty}\right\|^{2} \operatorname{Esup}_{\boldsymbol{\theta}}\left|\varepsilon_{t}^{4} \varepsilon_{t-i}^{4 s}\right|}<\infty$, $\operatorname{Esup}_{\boldsymbol{\theta}} R_{2 t}=O\left(\rho^{t}\right)$, so that $\frac{1}{\sqrt{T}} \sum_{t=1}^{T} \operatorname{Esup}_{\boldsymbol{\theta}} R_{2 t}=o(1)$. Thus, by Markov's inequality, $\frac{1}{\sqrt{T}} \sum_{t=1}^{T} \sup _{\boldsymbol{\theta}} R_{j t}=o_{p}(1), j=1,2$. Finally, note that

$$
\begin{aligned}
\frac{1}{\sqrt{T}} \sum_{t=1}^{T} \sup _{\boldsymbol{\theta}} R_{3 t} & \leq \sqrt{\frac{1}{T} \sum_{t=1}^{T} \sup _{\boldsymbol{\theta}} \varepsilon_{t}^{4}\left\|\mathbf{r}_{t}^{\infty}-\mathbf{r}_{t}\right\|^{2} \sum_{t=1}^{T} \sup _{\boldsymbol{\theta}}\left|\frac{h_{t}^{\infty}-h_{t}}{h_{t}^{\infty}}\right|^{2}} \\
& \leq\left\{\frac{1}{\sqrt{T}} \sum_{t=1}^{T} \sup _{\boldsymbol{\theta}} \varepsilon_{t}^{2}\left\|\mathbf{r}_{t}^{\infty}-\mathbf{r}_{t}\right\|\right\}\left\{\sum_{t=1}^{T} \sup _{\boldsymbol{\theta}}\left|\frac{h_{t}^{\infty}-h_{t}}{h_{t}^{\infty}}\right|\right\}
\end{aligned}
$$

because $\sum_{t=1}^{T} z_{t}^{2} \leq\left\{\sum_{t=1}^{T} z_{t}\right\}^{2}$ when $z_{t} \geq 0$ for all $t$.

Now, $\sum_{t=1}^{T} \operatorname{Esup}_{\boldsymbol{\theta}} \varepsilon_{t}^{2}\left\|\mathbf{r}_{t}^{\infty}-\mathbf{r}_{t}\right\|=O(1)$, by assumption, and $\operatorname{Esup}_{\boldsymbol{\theta}}\left|\left(h_{t}^{\infty}-h_{t}\right) / h_{t}^{\infty}\right|=$ $O\left(\rho^{t}\right)$ by (A.3) and previous results, so that $\frac{1}{\sqrt{T}} \sum_{t=1}^{T} \sup _{\theta} R_{3 t}=o_{p}(1)$, and the result follows.

(c) In a similar manner to the proof of Proposition 7, we have

$$
\begin{aligned}
& \left\|\frac{\partial \mathbf{d}_{\boldsymbol{\pi} t}^{\infty}(\boldsymbol{\theta})}{\partial \boldsymbol{\varphi}^{\prime}}\right\| \leq K\left\{\left\|\varepsilon_{t} \frac{\partial m_{t}}{\partial \boldsymbol{\varphi}} \mathbf{r}_{t}^{\infty}\right\|+\left|\varepsilon_{t}^{2}+1\right|\left(\left\|\frac{1}{h_{t}^{\infty}} \frac{\partial h_{t}^{\infty}}{\partial \boldsymbol{\varphi}}\right\|\left\|\mathbf{r}_{t}^{\infty}\right\|+\left\|\frac{\partial \mathbf{r}_{t}^{\infty}}{\partial \boldsymbol{\varphi}^{\prime}}\right\|\right)\right\} \\
& \left\|\frac{\partial \mathbf{d}_{\boldsymbol{\pi} t}^{\infty}(\theta)}{\partial \boldsymbol{\eta}^{\prime}}\right\| \leq K\left\{\left|\varepsilon_{t}^{2}+1\right|\left(\left\|\frac{1}{h_{t}^{\infty}} \frac{\partial h_{t}^{\infty}}{\partial \boldsymbol{\eta}}\right\|\left\|\mathbf{r}_{t}^{\infty}\right\|+\left\|\frac{\partial \mathbf{r}_{t}^{\infty}}{\partial \boldsymbol{\eta}}\right\|\right)\right\} .
\end{aligned}
$$

Then because $h_{t}^{\infty} \geq \lambda>0$ for all $t$ and $\boldsymbol{\theta}$, using Cauchy-Schwarz inequality, the assumptions of Theorem 2, and previous results, $\operatorname{Esup}_{\boldsymbol{\theta}}\left\|\frac{\partial \mathbf{d}_{\boldsymbol{\pi} t}^{\infty}(\boldsymbol{\theta})}{\partial \boldsymbol{\varphi}^{\prime}}\right\|<\infty$ and $\operatorname{E\operatorname {sup}\theta }\left\|\frac{\partial \mathbf{d}_{\boldsymbol{\pi} t}^{\infty}(\boldsymbol{\theta})}{\partial \boldsymbol{\eta}^{\prime}}\right\|$ $<\infty$. These are sufficient for a ULLN to apply, ensuring $\sup _{\boldsymbol{\theta}}\left\|\mathbf{P}_{\boldsymbol{\pi} \boldsymbol{\theta} T}^{\infty}(\boldsymbol{\theta})-\mathbf{J}_{\boldsymbol{\pi} \boldsymbol{\theta}}(\boldsymbol{\theta})\right\|=$ $o_{p}(1)$. 
Finally we briefly show that the additional assumptions of Theorem 2 are satisfied for the test variables considered in this paper in Section 4.

First, consider the Lundbergh and Teräsvirta (2002) nonlinearity (scalar) test variable $r_{t}=\varepsilon_{t-k}^{3}=r_{t}^{\infty}$. Trivially, assumption (i) is satisfied.

For (ii) we require $\mathrm{E}\left[\varepsilon_{0 t}^{6}\right]<\infty$, which is stronger than Assumption B1.

For (iii), $\operatorname{Esup}_{\boldsymbol{\theta} \in \Theta}\left\|\varepsilon_{t}^{2}\left(\partial \mathbf{r}_{t}^{\infty} / \partial \boldsymbol{\varphi}\right)\right\|=3 \operatorname{Esup}_{\boldsymbol{\theta}}\left\|\varepsilon_{t}^{2} \varepsilon_{t-k}^{2}\left(\partial m_{t-k} / \partial \boldsymbol{\varphi}\right)\right\|<\infty$, by CauchySchwarz inequality. We can proceed similarly for the asymmetry test variable of $I_{t-1} \varepsilon_{t-1}$ but taking into account (19).

For the nonlinearity test variable, with $\left\|\mathbf{r}_{t}^{\infty}\right\|$ having typical element $r_{k t}^{\infty}=\frac{1}{h_{t}^{\infty}} \sum_{i=0}^{\infty}$ $\psi_{i} \varepsilon_{t-k-i}^{3}, k=1, \ldots, q$, and noting $h_{t}^{\infty} \geq \alpha_{0}+\psi_{i} \alpha_{k} \varepsilon_{t-k-i}^{2}$,

$$
\begin{aligned}
& \left|r_{k t}^{\infty}\right| \leq \frac{1}{\sqrt{\alpha_{k}}} \sum_{i=0}^{\infty} \frac{\sqrt{\psi_{i} \alpha_{k}}\left|\varepsilon_{t-k-i}\right|}{\alpha_{0}+\psi_{i} \alpha_{k} \varepsilon_{t-k-i}^{2}} \sqrt{\psi_{i}} \varepsilon_{t-k-i}^{2} \leq K \sum_{i=0}^{\infty} \rho^{i} \varepsilon_{t-k-i}^{2}, \\
& \left|r_{k t}^{\infty}-r_{k t}\right| \leq K \sum_{i=t}^{\infty} \rho^{i} \varepsilon_{t-k-i}^{2} \\
& \left\|\frac{\partial r_{k t}^{\infty}}{\partial \boldsymbol{\varphi}}\right\| \leq 3 \frac{1}{h_{t}^{\infty}} \sum_{i=0}^{\infty} \psi_{i} \varepsilon_{t-k-i}^{2}\left\|\nabla_{\varphi} \mathbf{f}_{t-k-i}\right\|+\frac{1}{h_{t}^{\infty}} \sum_{i=0}^{\infty} \psi_{i}\left\|\varepsilon_{t-k-i}^{3} \mathbf{c}_{t}^{\infty}\right\| \\
& \leq 3 \frac{1}{\sqrt{\alpha_{k}}} \sum_{i=0}^{\infty} \frac{\sqrt{\psi_{i} \alpha_{k}}\left|\varepsilon_{t-k-i}\right|}{\alpha_{0}+\psi_{i} \alpha_{k} \varepsilon_{t-k-i}^{2}} \sqrt{\psi_{i}}\left\|\varepsilon_{t-k-i} \nabla_{\varphi} \mathbf{f}_{t-k-i}\right\| \\
& +\frac{1}{\sqrt{\alpha_{k}}} \sum_{i=0}^{\infty} \frac{\sqrt{\psi_{i} \alpha_{k}}\left|\varepsilon_{t-k-i}\right|}{\alpha_{0}+\psi_{i} \alpha_{k} \varepsilon_{t-k-i}^{2}} \sqrt{\psi_{i}} \varepsilon_{t-k-i}^{2}\left\|\mathbf{c}_{t}^{\infty}\right\| \\
& \leq K\left\{\sum_{i=0}^{\infty} \rho^{i}\left\|\varepsilon_{t-k-i} \nabla_{\varphi} \mathbf{f}_{t-k-i}\right\|+\sum_{i=0}^{\infty} \rho^{i} \varepsilon_{t-k-i}^{2}\left\|\mathbf{c}_{t}^{\infty}\right\|\right\} \\
& \left\|\frac{\partial r_{k t}^{\infty}}{\partial \boldsymbol{\eta}}\right\| \leq K\left\{\sum_{i=1}^{\infty} i \rho^{i} \varepsilon_{t-k-i}^{2}+\sum_{i=0}^{\infty} \rho^{i} \varepsilon_{t-k-i}^{2}\left\|\mathbf{x}_{t}^{\infty}\right\|\right\} .
\end{aligned}
$$

It is then straightforward to show that assumptions (i)-(iii) are satisfied. We can proceed similarly for the asymmetry test variable $r_{t}^{\infty}=\frac{1}{h_{t}^{\infty}} \sum_{i=0}^{\infty} \psi_{i} I_{t-k} \varepsilon_{t-k-i}$ but taking into account (19).

Proof of Lemma 2. The proof is similar to that of Lemma 1. We can show that $\operatorname{Esup}_{\boldsymbol{\theta}}\left\|\mathbf{r}_{t}^{\infty} \mathbf{q}_{t}^{\infty \prime}\right\|<\infty$, so that $T^{-1} \sum_{t=1}^{T} \sup _{\boldsymbol{\theta}}\left\|\mathbf{r}_{t}^{\infty} \mathbf{q}_{t}^{\infty \prime}-\mathrm{E}\left[\mathbf{r}_{t}^{\infty} \mathbf{q}_{t}^{\infty \prime}\right]\right\|=o_{p}(1)$, by previous arguments. It remains to establish that $T^{-1} \sum_{t=1}^{T} \sup _{\boldsymbol{\theta}}\left\|\mathbf{r}_{t}^{\infty} \mathbf{q}_{t}^{\infty \prime}-\mathbf{r}_{t} \mathbf{q}_{t}^{\prime}\right\|=o_{p}$ (1), 
because this then ensures that $T^{-1} \sum_{t=1}^{T}\left(\mathbf{r}_{t} \mathbf{q}_{t}^{\prime}\right)_{\boldsymbol{\theta}=\hat{\boldsymbol{\theta}}}-\mathrm{E}\left[\mathbf{r}_{t}^{\infty} \mathbf{q}_{t}^{\infty \prime}\right]_{\boldsymbol{\theta}=\boldsymbol{\theta}_{0}}=o_{p}(1)$. Note that

$$
\begin{aligned}
& T^{-1} \sum_{t=1}^{T}\left\|\mathbf{r}_{t}^{\infty} \mathbf{q}_{t}^{\infty \prime}-\mathbf{r}_{t} \mathbf{q}_{t}^{\prime}\right\| \leq T^{-1} \sum_{t=1}^{T}\left\|\mathbf{r}_{t}^{\infty}-\mathbf{r}_{t}\right\|\left\|\mathbf{q}_{t}^{\infty}\right\|+T^{-1} \sum_{t=1}^{T}\left\|\mathbf{q}_{t}^{\infty}-\mathbf{q}_{t}\right\|\left\|\mathbf{r}_{t}^{\infty}\right\| \\
& +T^{-1} \sum_{t=1}^{T}\left\|\mathbf{r}_{t}^{\infty}-\mathbf{r}_{t}\right\|\left\|\mathbf{q}_{t}^{\infty}-\mathbf{q}_{t}\right\|
\end{aligned}
$$

and it can easily be shown that each term on the right-hand side is $o_{p}(1)$. We also know that $\hat{k}_{c}=k_{c}+o_{p}(1)$ and $\hat{v}_{c}=v_{c}+o_{p}(1)$, from the proof of Lemma 1, and the result follows.

\section{Proof of Lemma 3.}

(i) First, from the expression for $\frac{\partial d_{\eta t}^{\infty}(\boldsymbol{\theta})}{\partial \varphi^{\prime}}$ in the proof of Proposition 7, it is easy to see that $\mathbf{J}_{\eta \varphi}=\frac{1}{2} \mathrm{E}\left[\mathbf{x}_{t}^{\infty} \mathbf{c}_{t}^{\infty \prime}\right]_{\boldsymbol{\theta}=\boldsymbol{\theta}_{0}}$. Now,

$\mathrm{E}\left[\mathbf{x}_{t}^{\infty} \mathbf{c}_{t}^{\infty \prime}\right]_{\boldsymbol{\theta}=\boldsymbol{\theta}_{0}}$

$$
=-2 \sum_{k=1}^{q} \alpha_{0 k} \mathrm{E}\left[\frac{1}{h_{t}^{\infty 2}} \sum_{i=0}^{\infty} \sum_{j=0}^{\infty} \psi_{i} \psi_{j} \mathbf{s}_{t-1-i}^{\infty} \varepsilon_{t-k-j} \mathbf{f}_{t-k-j}^{\prime}\right]_{\boldsymbol{\theta}=\boldsymbol{\theta}_{0}},
$$

which exists, provided $\mathrm{E}\left[\left(1 / h_{t}^{\infty 2}\right) \varepsilon_{t-l} \mathbf{s}_{t-m}^{\infty} \mathbf{f}_{t-l}^{\prime}\right] \boldsymbol{\theta}=\boldsymbol{\theta}_{0}$ exists (for all $l, m$ ), because $\left|\sum_{i=0}^{\infty} \sum_{j=0}^{\infty} \psi_{i} \psi_{j}\right|=\left|\sum_{i=0}^{\infty} \psi_{i}\right|^{2} \leq\left\{\sum_{i=0}^{\infty}\left|\psi_{i}\right|\right\}^{2}<\infty$.

Thus $\mathrm{E}\left[\left(1 / h_{t}^{\infty 2}\right) \varepsilon_{t-l} \mathbf{s}_{t-m}^{\infty} \mathbf{f}_{t-l}^{\prime}\right]_{\boldsymbol{\theta}=\boldsymbol{\theta}_{0}}$ has to be examined for the cases $l=m$, $l<m$, and $l>m$, where $\mathbf{s}_{t-m}^{\infty}=\left(1, \varepsilon_{t-m}^{2}, \ldots, \varepsilon_{t-m-q+1}^{2}, h_{t-m}^{\infty}, \ldots, h_{t-m-p+1}^{\infty}\right)^{\prime}$. Specifically, for $l=m, \mathrm{E}\left[\left(1 / h_{t}^{\infty 2}\right) \varepsilon_{t-l} \mathbf{s}_{t-l}^{\infty} \mathbf{f}_{t-l}^{\prime}\right]_{\boldsymbol{\theta}=\boldsymbol{\theta}_{0}}$ is

$$
\begin{aligned}
\mathrm{E}\left\{\mathrm { E } \left[\frac { 1 } { h _ { t } ^ { \infty 2 } } \left(\varepsilon_{t-l}, \varepsilon_{t-l}^{3}, \ldots, \varepsilon_{t-l} \varepsilon_{t-l-q+1}^{2},\right.\right.\right. & \\
& \left.\left.\left.\varepsilon_{t-l} h_{t-l}^{\infty}, \ldots, \varepsilon_{t-l} h_{t-l-p+1}^{\infty}\right)^{\prime} \mid \mathcal{F}_{t-l-1}\right] \mathbf{f}_{t-l}^{\prime}\right\}_{\boldsymbol{\theta}=\boldsymbol{\theta}_{0}},
\end{aligned}
$$

which is zero if the preceding expression for the conditional expectation, given $\mathcal{F}_{t-l-1}$, is zero. To establish the latter, follow Engle (1982) and treat this conditional expectation in two steps, observing that $\varepsilon_{t-l-n}, n=1,2, \ldots$, are $\mathcal{F}_{t-l-1}$ measurable. First, construct the conditional expectation given $\mathcal{F}_{t-l}$, which is

$$
\begin{aligned}
& {\left[\left(\varepsilon_{t-l}, \varepsilon_{t-l}^{3}, \ldots, \varepsilon_{t-l} \varepsilon_{t-l-q+1}^{2}, \varepsilon_{t-l} h_{t-l}^{\infty}, \ldots, \varepsilon_{t-l} h_{t-l-p+1}^{\infty}\right)^{\prime}\right.} \\
& \left.\quad \times \mathrm{E}\left\{\frac{1}{h_{t}^{\infty 2}} \mid \mathcal{F}_{t-l}\right\}\right]_{\boldsymbol{\theta}=\boldsymbol{\theta}_{0}} \equiv \phi\left(\varepsilon_{t-l}\right),
\end{aligned}
$$


where it is implicit that $\phi(\cdot)$ is evaluated at $\boldsymbol{\theta}=\boldsymbol{\theta}_{0}$. Because $h_{t}^{\infty}$ is symmetric in $\varepsilon_{t-l}$ and the elements in $\varepsilon_{t-l} s_{t-l}^{\infty}$ are all antisymmetric in $\varepsilon_{t-l}$, the elements in $\left(h_{t}^{\infty}\right)^{-2} \varepsilon_{t-l} s_{t-l}^{\infty}$ are antisymmetric in $\varepsilon_{t-l}$, which forms part of $\mathcal{F}_{t-l}$, and, at the second step, expectations with respect to $\mathcal{F}_{t-l-1}$ are taken only with random elements. Now, because $h_{t}^{\infty}$ is symmetric in $\varepsilon_{t-l}$, its conditional density given $\varepsilon_{t-l}$ is also symmetric in $\varepsilon_{t-l}$. Therefore, by Engle $\left(1982\right.$, lem., p. 1006), $\phi\left(\varepsilon_{t-l}\right)$ is antisymmetric in $\varepsilon_{t-l}$. Finally, the second step involves $\mathrm{E}\left[\phi\left(\varepsilon_{t-l}\right) \mid \mathcal{F}_{t-l-1}\right]$, which is zero, because the conditional density of $\varepsilon_{t-l}$ given $\mathcal{F}_{t-l-1}$ is symmetric and $\phi(\cdot)$ is antisymmetric.

The other typical expectation in (B.1) for $l<m$ and $l>m$ is

$\mathrm{E}\left\{\mathrm{E}\left[\frac{1}{h_{t}^{\infty 2}} \varepsilon_{t-m} \mathbf{s}_{t-l}^{\infty} \mid \mathcal{F}_{t-m-1}\right] \mathbf{f}_{t-m}^{\prime}\right\}_{\boldsymbol{\theta}=\boldsymbol{\theta}_{0}}$,

which is zero if the conditional expectation, given $\mathcal{F}_{t-m-1}$, is zero. The latter can be expressed as

$\mathrm{E}\left[\frac{1}{h_{t}^{\infty 2}} \varepsilon_{t-m} \mathbf{s}_{t-l}^{\infty} \mid \mathcal{F}_{t-m-1}\right]_{\boldsymbol{\theta}=\boldsymbol{\theta}_{0}}$
$\quad=\mathrm{E}\left\{\mathrm{E}\left[\frac{1}{h_{t}^{\infty 2}} \varepsilon_{t-m} \mathbf{s}_{t-l}^{\infty} \mid \mathcal{F}_{t-m}\right] \mid \mathcal{F}_{t-m-1}\right\}_{\boldsymbol{\theta}=\boldsymbol{\theta}_{0}}$.

For $l>m$, the elements of $\mathbf{s}_{t-l}^{\infty}$ belong to $\mathcal{F}_{t-m-1}$, and the preceding arguments show that $\mathrm{E}\left[\left(h_{t}^{\infty}\right)^{-2} \varepsilon_{t-m} \mid \mathcal{F}_{t-m-1}\right]_{\boldsymbol{\theta}=\boldsymbol{\theta}_{0}}=0$. For $m>l$, note that the elements of $\left(h_{t}^{\infty}\right)^{-2} \mathbf{s}_{t-l}^{\infty}$ are symmetric in $\varepsilon_{t-m}$, so that $\mathrm{E}\left[\left(h_{t}^{\infty}\right)^{-2} \varepsilon_{t-m} \mathbf{s}_{t-l}^{\infty} \mid \mathcal{F}_{t-m}\right]_{\boldsymbol{\theta}=\boldsymbol{\theta}_{0}} \equiv$ $\phi\left(\varepsilon_{t-m}\right)$ is antisymmetric in $\varepsilon_{t-m}$ and, again, $\mathrm{E}\left[\phi\left(\varepsilon_{t-m}\right) \mid \mathcal{F}_{t-m-1}\right]_{\boldsymbol{\theta}=\boldsymbol{\theta}_{0}}=0$, where elements included in the conditioning set $\mathcal{F}_{t-m-1}$ are treated as nonrandom when taking the conditional expectation. It follows that $\mathbf{J}_{\eta \varphi}=\mathbf{0}$.

Because $v_{c}=0$, (ii)-(iv) follow immediately, given previous definitions.

Proof of Lemma 4. Note that $\mathbf{c}_{t}^{\infty \prime}=\left(h_{t}^{\infty}\right)^{-1} \sum_{i=0}^{\infty} \beta_{1}^{i} \varepsilon_{t-1-i} \mathbf{f}_{t-1-i}^{\prime}$ and $\mathbf{r}_{t}=\left(\varepsilon_{t-1}\right.$, $\left.\varepsilon_{t-1}^{3}\right)^{\prime}$, so that $\mathbf{J}_{\boldsymbol{\pi} \varphi}$ can be written as

$$
\begin{aligned}
\mathbf{J}_{\boldsymbol{\pi} \boldsymbol{\varphi}}=-2 \alpha_{01} \mathrm{E}\left\{\mathrm{E}\left[\frac{1}{h_{t}^{\infty}}\left(\begin{array}{c}
\varepsilon_{t-1}^{2} \\
\varepsilon_{t-1}^{4}
\end{array}\right) \mid \mathcal{F}_{t-2}\right] \mathbf{f}_{t-1}^{\prime}\right. \\
\left.+\sum_{i=1}^{\infty} \beta_{1}^{i} \mathrm{E}\left[\frac{1}{h_{t}^{\infty}}\left(\begin{array}{c}
\varepsilon_{t-1} \\
\varepsilon_{t-1}^{3}
\end{array}\right) \varepsilon_{t-1-i} \mid \mathcal{F}_{t-2}\right] \mathbf{f}_{t-1-i}^{\prime}\right\}_{\boldsymbol{\theta}=\boldsymbol{\theta}_{0}} \\
=-2 \alpha_{01} \mathrm{E}\left\{\mathrm{E}\left[\frac{1}{h_{t}^{\infty}}\left(\begin{array}{c}
\varepsilon_{t-1}^{2} \\
\varepsilon_{t-1}^{4}
\end{array}\right) \mid \mathcal{F}_{t-2}\right] \mathbf{f}_{t-1}^{\prime}\right\}_{\boldsymbol{\theta}=\boldsymbol{\theta}_{0}}
\end{aligned}
$$

which is nonzero, in general, because $\mathrm{E}\left[\frac{1}{h_{t}^{\infty}}\left(\begin{array}{c}\varepsilon_{t-1}^{2} \\ \varepsilon_{t-1}^{4}\end{array}\right) \mid \mathcal{F}_{t-2}\right]_{\boldsymbol{\theta}=\boldsymbol{\theta}_{0}}>0$, a.s. The second term (after the second equality) is zero because, for $j \geq 2$, 
$\mathrm{E}\left[\frac{1}{h_{t}^{\infty}}\left(\begin{array}{c}\varepsilon_{t-1} \\ \varepsilon_{t-1}^{3}\end{array}\right) \varepsilon_{t-j} \mid \mathcal{F}_{t-2}\right]_{\boldsymbol{\theta}=\boldsymbol{\theta}_{0}}=\left(\varepsilon_{t-j} \mathrm{E}\left[\frac{1}{h_{t}^{\infty}}\left(\begin{array}{c}\varepsilon_{t-1} \\ \varepsilon_{t-1}^{3}\end{array}\right) \mid \mathcal{F}_{t-2}\right]\right)_{\boldsymbol{\theta}=\boldsymbol{\theta}_{0}}$

and

$\mathrm{E}\left[\frac{1}{h_{t}^{\infty}} \varepsilon_{t-1}^{s} \mid \mathcal{F}_{t-2}\right]_{\boldsymbol{\theta}=\boldsymbol{\theta}_{0}}=\mathrm{E}\left[\phi\left(\varepsilon_{t-1}\right) \mid \mathcal{F}_{t-2}\right]_{\boldsymbol{\theta}=\boldsymbol{\theta}_{0}}$,

where $\mathrm{E}\left[\left(h_{t}^{\infty}\right)^{-1} \varepsilon_{t-1}^{s} \mid \mathcal{F}_{t-1}\right]=\phi\left(\varepsilon_{t-1}\right), s=1,3$, which is antisymmetric in $\varepsilon_{t-1}$, so that $\mathrm{E}\left[\phi\left(\varepsilon_{t-1}\right) \mid \mathcal{F}_{t-2}\right]=0$ because the conditional density of $\varepsilon_{t-1}$ given $\mathcal{F}_{t-2}$ is symmetric. Thus, in general, $\mathbf{J}_{\boldsymbol{\pi} \varphi} \neq \mathbf{0}$.

Second, with $\mathbf{x}_{t}^{\infty \prime}=\left(h_{t}^{\infty}\right)^{-1} \sum_{i=0}^{\infty} \beta_{1}^{i} \mathbf{s}_{t-1-i}^{\infty \prime}, \mathbf{J}_{\pi \boldsymbol{\eta}}$ can be written as

$$
\begin{aligned}
\mathbf{J}_{\pi \boldsymbol{\eta}}=\mathrm{E}\left\{\mathrm{E}\left[\frac{1}{h_{t}^{\infty}}\left(\begin{array}{c}
\varepsilon_{t-1} \\
\varepsilon_{t-1}^{3}
\end{array}\right) \mathbf{s}_{t-1}^{\infty \prime} \mid \mathcal{F}_{t-2}\right]\right. \\
\left.+\sum_{i=1}^{\infty} \beta_{1}^{i} \mathrm{E}\left[\frac{1}{h_{t}^{\infty}}\left(\begin{array}{c}
\varepsilon_{t-1} \\
\varepsilon_{t-1}^{3}
\end{array}\right) \mid \mathcal{F}_{t-2}\right] \mathbf{s}_{t-1-i}^{\infty \prime}\right\}_{\boldsymbol{\theta}=\boldsymbol{\theta}_{0}} .
\end{aligned}
$$

Similar arguments to those employed previously imply that $\mathbf{J}_{\boldsymbol{\pi} \boldsymbol{\eta}}$ is the null vector.

Proof of Proposition 1. The method of proof follows very closely that of Godfrey (1996). Consider the negative size bias test of Engle and $\mathrm{Ng}$ (1993) in which $\hat{r}_{t}=\hat{I}_{t-1} \hat{\varepsilon}_{t-1}$ and for simplicity, in this case, $m\left(\mathbf{w}_{t} ; \boldsymbol{\varphi}\right)=\mathbf{w}_{t}^{\prime} \boldsymbol{\varphi}$. Define the following dummy variables, which will be employed in the ensuing asymptotic analysis:

$\mathcal{D}_{t 1}=1, \quad$ if $\quad \varepsilon_{0, t-1} \leq 0 \quad$ and $\quad \hat{\varepsilon}_{t-1} \leq 0, \quad \mathcal{D}_{t 1}=0, \quad$ otherwise,

$\mathcal{D}_{t 2}=1, \quad$ if $\quad \varepsilon_{0, t-1}>0 \quad$ and $\quad \hat{\varepsilon}_{t-1} \leq 0, \quad \mathcal{D}_{t 2}=0, \quad$ otherwise,

$\mathcal{D}_{t 3}=1, \quad$ if $\quad \varepsilon_{0, t-1} \leq 0 \quad$ and $\quad \hat{\varepsilon}_{t-1}>0, \quad \mathcal{D}_{t 3}=0, \quad$ otherwise,

$\mathcal{D}_{t 4}=1, \quad$ if $\quad \varepsilon_{0, t-1}>0 \quad$ and $\quad \hat{\varepsilon}_{t-1}>0, \quad \mathcal{D}_{t 4}=0, \quad$ otherwise

for $t=1, \ldots, T$. Note that both $\operatorname{Pr}\left(\mathcal{D}_{t 2}=1\right)$ and $\operatorname{Pr}\left(\mathcal{D}_{t 3}=1\right)$ tend to zero as $T \rightarrow \infty$, under fairly general conditions on $\mathbf{w}_{t}$, because $\hat{\varepsilon}_{t-1}-\varepsilon_{0, t-1}=-\mathbf{w}_{t-1}^{\prime}\left(\hat{\boldsymbol{\varphi}}-\boldsymbol{\varphi}_{0}\right)$ and $\hat{\boldsymbol{\varphi}}$ is $\sqrt{T}$-consistent for $\varphi_{0}$.

Then, noting that $\hat{r}_{t}-r_{0 t}=0$ when $\mathcal{D}_{t 4}=1$, the difference between $\sqrt{T} D_{\boldsymbol{\pi} T}(\hat{\boldsymbol{\theta}})$ and $\frac{1}{\sqrt{T}} \sum_{t=1}^{T}\left[\left(\frac{\hat{\varepsilon}_{t}^{2}}{\hat{h}_{t}}-1\right) r_{0 t}\right]$ can be expressed as

$$
\begin{aligned}
\frac{1}{\sqrt{T}} & \sum_{t=1}^{T}\left[\left(\frac{\hat{\varepsilon}_{t}^{2}}{\hat{h}_{t}}-1\right)\left(\hat{r}_{t}-r_{0 t}\right)\right] \\
& =\frac{1}{\sqrt{T}} \sum_{t=1}^{T}\left[\left(\frac{\hat{\varepsilon}_{t}^{2}}{\hat{h}_{t}}-1\right)\left\{\mathcal{D}_{t 1}\left(\hat{\varepsilon}_{t-1}-\varepsilon_{0, t-1}\right)+\mathcal{D}_{t 2} \hat{\varepsilon}_{t-1}-\mathcal{D}_{t 3} \varepsilon_{0, t-1}\right\}\right] \\
& =\Gamma_{1}+\Gamma_{2}+\Gamma_{3}
\end{aligned}
$$


where

$$
\begin{aligned}
\Gamma_{1} & =\frac{1}{\sqrt{T}} \sum_{t=1}^{T}\left[\mathcal{D}_{t 1}\left(\frac{\hat{\varepsilon}_{t}^{2}}{\hat{h}_{t}}-1\right)\left(\hat{\varepsilon}_{t-1}-\varepsilon_{0, t-1}\right)\right] \\
& =-\frac{1}{T} \sum_{t=1}^{T}\left[\mathcal{D}_{t 1}\left(\frac{\hat{\varepsilon}_{t}^{2}}{\hat{h}_{t}}-1\right) \mathbf{w}_{t-1}^{\prime}\right] \sqrt{T}\left(\hat{\boldsymbol{\varphi}}-\boldsymbol{\varphi}_{0}\right), \\
\Gamma_{2} & =\frac{1}{\sqrt{T}} \sum_{t=1}^{T}\left[\mathcal{D}_{t 2}\left(\frac{\hat{\varepsilon}_{t}^{2}}{\hat{h}_{t}}-1\right) \hat{\varepsilon}_{t-1}\right] \\
\Gamma_{3} & =-\frac{1}{\sqrt{T}} \sum_{t=1}^{T}\left[\mathcal{D}_{t 3}\left(\frac{\hat{\varepsilon}_{t}^{2}}{\hat{h}_{t}}-1\right) \varepsilon_{0, t-1}\right]
\end{aligned}
$$

are all $o_{p}(1)$, for $j=1,2,3$, implying $\frac{1}{\sqrt{T}} \sum_{t=1}^{T}\left[\left(\left(\hat{\varepsilon}_{t}^{2} / \hat{h}_{t}\right)-1\right)\left(\hat{r}_{t}-r_{0 t}\right)\right]=o_{p}(1)$. For example, $\Gamma_{3}$ can be written as

$$
\begin{aligned}
\Gamma_{3} & =-\left(\sum_{t=1}^{T} \mathcal{D}_{t 3} / T\right)^{1 / 2}\left[\left(\sum_{t=1}^{T} \mathcal{D}_{t 3}\right)^{-1 / 2} \sum_{t=1}^{T} \mathcal{D}_{t 3}\left(\frac{\hat{\varepsilon}_{t}^{2}}{\hat{h}_{t}}-1\right) \varepsilon_{0, t-1}\right] \\
& =-\left(\mathcal{M}_{3} / T\right)^{1 / 2}\left[\left(\mathcal{M}_{3}\right)^{-1 / 2} \sum_{t \in \mathcal{T}_{3}}\left(\frac{\hat{\varepsilon}_{t}^{2}}{\hat{h}_{t}}-1\right) \varepsilon_{0, t-1}\right]
\end{aligned}
$$

where $\mathcal{M}_{3}=\sum_{t=1}^{T} \mathcal{D}_{t 3}$ is the number of observations for which $\mathcal{D}_{t 3}=1$ and $\mathcal{T}_{3}$ denotes the subsample of observations with $\mathcal{D}_{t 3}=1$. Now, $\mathcal{M}_{3} / T$ is the proportion of sample observations for which $\mathcal{D}_{t 3}=1$. Because $\operatorname{Pr}\left(\mathcal{D}_{t 3}=1\right) \rightarrow 0, \mathcal{M}_{3} / T$ is thus $o_{p}(1)$.

Similar to the preceding analysis, because $\mathcal{M}_{3} \rightarrow \infty$, a mean value expansion of $\left(\mathcal{M}_{3}\right)^{-1 / 2} \sum_{t \in \mathcal{T}_{3}}\left(\frac{\hat{\varepsilon}_{t}^{2}}{\hat{h}_{t}}-1\right) \varepsilon_{0, t-1}$ reveals that it is $O_{p}(1)$. Therefore, $\Gamma_{3}=o_{p}(1)$, and, in a similar fashion, it can be shown that $\Gamma_{2}=o_{p}(1)$.

Turning to $\Gamma_{1}$,

$$
\Gamma_{1}=-\left(\mathcal{M}_{1} / T\right)\left[\mathcal{M}_{1}^{-1} \sum_{t \in \mathcal{T}_{1}}\left(\frac{\hat{\varepsilon}_{t}^{2}}{\hat{h}_{t}}-1\right) \mathbf{w}_{t-1}^{\prime}\right] \sqrt{T}\left(\hat{\boldsymbol{\varphi}}-\boldsymbol{\varphi}_{0}\right)
$$

where $\mathcal{M}_{1}=\sum_{t=1}^{T} \mathcal{D}_{t 1}$ is the number of observations for which $\mathcal{D}_{t 1}=1$ and $\mathcal{T}_{1}$ denotes the subsample of observations with $\mathcal{D}_{t 1}=1$. Now, $\mathcal{M}_{1} / T$ is the proportion of sample observations for which $\mathcal{D}_{t 1}=1$. In this case, $\operatorname{Pr}\left(\mathcal{D}_{t 1}=1\right) \rightarrow 1$, so that $\mathcal{M}_{1} / T \stackrel{p}{\rightarrow} 1$, and a mean value expansion of $\mathcal{M}_{1}^{-1} \Sigma_{t \in \mathcal{T}_{1}}\left(\left(\hat{\varepsilon}_{t}^{2} / \hat{h}_{t}\right)-1\right) \mathbf{w}_{t-1}^{\prime}$ reveals that it is $o_{p}(1)$. Hence, $\Gamma_{1}=o_{p}(1)$, also. 
Proof of Lemma 5. Specifically, $\mathbf{J}_{\pi \varphi}$ can be written as

$$
\begin{aligned}
\mathbf{J}_{\pi \varphi}=-2 \alpha_{01} \mathrm{E}\left\{\mathrm{E}\left[\frac{1}{h_{t}^{\infty}} I_{t-1} \varepsilon_{t-1}^{2} \mid \mathcal{F}_{t-2}\right] \mathbf{f}_{t-1}^{\prime}\right. \\
\left.+\sum_{i=1}^{\infty} \beta_{1}^{i} \mathrm{E}\left[\frac{1}{h_{t}^{\infty}} I_{t-1} \varepsilon_{t-1} \varepsilon_{t-1-i} \mid \mathcal{F}_{t-2}\right] \mathbf{f}_{t-1-i}^{\prime}\right\}_{\boldsymbol{\theta}=\boldsymbol{\theta}_{0}},
\end{aligned}
$$

which is nonzero (certainly, $\mathrm{E}\left[\left(1 / h_{t}^{\infty}\right) I_{t-1} \varepsilon_{t-1}^{2} \mid \mathcal{F}_{t-2}\right]$ is nonnegative).

Proof of Lemma 6. The proof is similar to that of Lemma 3. First, for nonnegligible estimation effects from the conditional mean,

$$
\mathbf{J}_{\pi \boldsymbol{\varphi}}=-2 \sum_{k=1}^{q} \alpha_{0 k} \mathrm{E}\left[\frac{1}{h_{t}^{\infty 2}} \sum_{i=0}^{\infty} \sum_{j=0}^{\infty} \psi_{i} \psi_{j} \varepsilon_{t-k-j} \mathbf{v}_{t-1-i} \mathbf{f}_{t-k-j}^{\prime}\right]_{\boldsymbol{\theta}=\boldsymbol{\theta}_{0}}
$$

is nonzero, in general, if at least one element in $\mathbf{J}_{\pi \varphi}$ is nonzero. This amounts to examining the typical expectation $\mathrm{E}\left[\frac{1}{h_{t}^{\infty 2}} \varepsilon_{t-l}^{s} \varepsilon_{t-m} \mathbf{f}_{t-m}^{\prime}\right]_{\boldsymbol{\theta}=\boldsymbol{\theta}_{0}}$ for $l=m, l<m$, and $l>m$, where $s=1,3$. First, for $l=m$,

$\mathrm{E}\left[\frac{1}{h_{t}^{\infty 2}} \varepsilon_{t-l}^{s} \varepsilon_{t-l} \mathbf{f}_{t-l}^{\prime}\right]_{\boldsymbol{\theta}=\boldsymbol{\theta}_{0}}=\mathrm{E}\left\{\mathrm{E}\left[\frac{1}{h_{t}^{\infty 2}} \varepsilon_{t-l}^{s} \varepsilon_{t-l} \mid \mathcal{F}_{t-l-1}\right] \mathbf{f}_{t-l}^{\prime}\right\}_{\boldsymbol{\theta}=\boldsymbol{\theta}_{0}}$

with the conditional expectation given by $\mathrm{E}\left[\left(h_{t}^{\infty}\right)^{-2} \varepsilon_{t-l}^{u} \mid \mathcal{F}_{t-l-1}\right]$ for $u=2,4$.

Similar to the arguments in Lemma $4, \mathrm{E}\left[\left(h_{t}^{\infty}\right)^{-2} \varepsilon_{t-l}^{u} \mid \mathcal{F}_{t-l-1}\right]>0$, a.s., and thus $\mathrm{E}\left[\left(1 / h_{t}^{\infty 2}\right) \varepsilon_{t-l}^{s} \varepsilon_{t-l} \mathbf{f}_{t-l}^{\prime}\right]_{\boldsymbol{\theta}=\boldsymbol{\theta}_{0}}$ is nonzero. Further, for $l<m$,

$$
\begin{gathered}
\mathrm{E}\left\{\mathbf{f}_{t-m}^{\prime} \mathrm{E}\left[\frac{1}{h_{t}^{\infty 2}} \varepsilon_{t-l}^{s} \varepsilon_{t-m} \mid \mathcal{F}_{t-m-1}\right]\right\}_{\boldsymbol{\theta}=\boldsymbol{\theta}_{0}} \\
=\mathrm{E}\left\{\mathbf{f}_{t-m}^{\prime} \mathrm{E}\left[\varepsilon_{t-m} \mathrm{E}\left[\frac{1}{h_{t}^{\infty 2}} \varepsilon_{t-l}^{s} \mid \mathcal{F}_{t-l-1}\right] \mid \mathcal{F}_{t-m-1}\right]\right\}_{\boldsymbol{\theta}=\boldsymbol{\theta}_{0}},
\end{gathered}
$$

and arguments similar to those employed in the proof of Lemma 3 establish that $\mathrm{E}\left[\left(h_{t}^{\infty}\right)^{-2} \times\right.$ $\left.\varepsilon_{t-l}^{s} \mid \mathcal{F}_{t-l-1}\right]=0$ for $s=1,3$. For $l>m$, because $\mathcal{F}_{t-l-1} \subseteq \mathcal{F}_{t-m-1}$,

$\mathrm{E}\left[\frac{1}{h_{t}^{\infty 2}} \varepsilon_{t-l}^{s} \varepsilon_{t-m} \mathbf{f}_{t-m}^{\prime}\right]_{\boldsymbol{\theta}=\boldsymbol{\theta}_{0}}=\mathrm{E}\left\{\mathbf{f}_{t-m}^{\prime} \varepsilon_{t-l}^{s} \mathrm{E}\left[\frac{1}{h_{t}^{\infty 2}} \varepsilon_{t-m} \mid \mathcal{F}_{t-m-1}\right]\right\}_{\boldsymbol{\theta}=\boldsymbol{\theta}_{0}}$,

where the elements $\varepsilon_{t-l}^{s}, s=1,3$ belong to $\mathcal{F}_{t-m-1}$ and previous arguments show that $\mathrm{E}\left[\left(h_{t}^{\infty}\right)^{-2} \varepsilon_{t-m} \mid \mathcal{F}_{t-m-1}\right]_{\boldsymbol{\theta}=\boldsymbol{\theta}_{0}}=0$.

Second, for $\mathbf{J}_{\boldsymbol{\pi} \boldsymbol{\eta}}$, the expectation to be examined is $\mathrm{E}\left[\left(1 / h_{t}^{\infty 2}\right) \varepsilon_{t-l}^{s} \mathbf{s}_{t-m}^{\infty \prime}\right]_{\boldsymbol{\theta}=\boldsymbol{\theta}_{0}}, s=1,3$ for $l=m, l>m$, and $l<m$. Arguments similar to those used in the proof of Lemma 
3(i) show that $\mathbf{J}_{\boldsymbol{\pi} \boldsymbol{\eta}}=\mathbf{0}$. In particular, notice that for $s=1$, the preceding expectation was shown to be zero for all three cases in Lemma 3. Similar arguments also apply for $s=3$.

Proof of Lemma 7. First, with $\mathbf{c}_{t}^{\infty \prime}=\left(h_{t}^{\infty}\right)^{-1} \sum_{i=0}^{\infty} \psi_{i} \varepsilon_{t-1-i} \mathbf{f}_{t-1-i}^{\prime}$ and $\mathbf{r}_{t}^{\infty}=$ $\left(h_{t}^{\infty}\right)^{-1} \sum_{i=0}^{\infty} \psi_{i} \mathbf{v}_{t-1-i}, \mathbf{J}_{\boldsymbol{\pi} \varphi}$ can be written as

$\mathbf{J}_{\pi \varphi}=-2 \sum_{k=1}^{q} \alpha_{0 k} \mathrm{E}\left[\frac{1}{h_{t}^{\infty 2}} \sum_{i=0}^{\infty} \sum_{j=0}^{\infty} \psi_{i} \psi_{j} \varepsilon_{t-k-j} \mathbf{v}_{t-1-i} \mathbf{f}_{t-k-j}^{\prime}\right]_{\boldsymbol{\theta}=\boldsymbol{\theta}_{0}}$.

For a typical element in $\mathbf{J}_{\boldsymbol{\pi} \varphi}$, the expectation to be examined is $\mathrm{E}\left[\left(1 / h_{t}^{\infty 2}\right) I_{t-l} \varepsilon_{t-l} \varepsilon_{t-m} \times\right.$ $\left.\mathbf{f}_{t-m}^{\prime}\right]$ for the cases $l=m, l<m$, and $l>m$. Consider just $l=m$; here we have $\mathrm{E}\left[\left(1 / h_{t}^{\infty 2}\right) \times\right.$ $\left.I_{t-l} \varepsilon_{t-l}^{2} \mathbf{f}_{t-l}^{\prime}\right]_{\boldsymbol{\theta}=\theta_{0}}=\mathrm{E}\left\{\mathrm{E}\left[\left(1 / h_{t}^{2}\right) I_{t-l} \varepsilon_{t-l}^{2} \mid \mathcal{F}_{t-l-1}\right] \mathbf{f}_{t-l}^{\prime}\right\}_{\boldsymbol{\theta}=\boldsymbol{\theta}_{0}}$, which is certainly nonzero. Second, with $\mathbf{x}_{t}^{\infty \prime}=\left(h_{t}^{\infty}\right)^{-1} \sum_{i=0}^{\infty} \psi_{i} \mathbf{s}_{t-1-i}^{\infty \prime}, \mathbf{J}_{\pi \boldsymbol{\eta}}$ in (11) can be written as

$\mathbf{J}_{\pi \boldsymbol{\eta}}=\mathrm{E}\left[\frac{1}{h_{t}^{\infty 2}} \sum_{i=0}^{\infty} \sum_{j=0}^{\infty} \psi_{i} \psi_{j} \mathbf{v}_{t-1-i} \mathbf{s}_{t-1-j}^{\infty \prime}\right]_{\boldsymbol{\theta}=\boldsymbol{\theta}_{0}}$.

For a typical element in $\mathbf{J}_{\boldsymbol{\pi} \boldsymbol{\eta}}$, the expectation to be examined is $\mathrm{E}\left[\frac{1}{h_{t}^{\infty 2}} I_{t-l} \varepsilon_{t-l} \mathbf{s}_{t-m}^{\infty \prime}\right]$ for the cases $l=m, l<m$, and $l>m$. Arguments similar to those employed previously show that this is nonzero in general. 